\title{
Coherence Techniques at Extreme Ultraviolet Wavelengths
}

\author{
by \\ Chang Chang \\ B.S. (National Taiwan University) 1996 \\ M.Eng. (Cornell University) 1997 \\ A dissertation submitted in partial satisfaction of the \\ requirements for the degree of \\ Doctor of Philosophy \\ in \\ Engineering-Electrical Engineering and Computer Sciences \\ in the \\ GRADUATE DIVISION \\ of the \\ UNIVERSITY of CALIFORNIA, BERKELEY
}

\author{
Committee in charge: \\ Professor David Attwood, Chair \\ Professor Raymond Y. Chiao \\ Professor W. Jack Welch
}

Fall 2002 
The dissertation of Chang Chang is approved:

\begin{tabular}{lr}
\hline Chair & Date \\
& \\
\hline Date
\end{tabular}

University of California at Berkeley

2002 
Coherence Techniques at Extreme Ultraviolet Wavelengths

Copyright 2002

by

Chang Chang 


\author{
Abstract \\ Coherence Techniques at Extreme Ultraviolet Wavelengths \\ by \\ Chang Chang \\ Doctor of Philosophy in Engineering-Electrical Engineering and Computer Sciences \\ University of California, Berkeley \\ Professor David Attwood, Chair
}

The renaissance of Extreme Ultraviolet (EUV) and soft x-ray (SXR) optics in recent years is mainly driven by the desire of printing and observing ever smaller features, as in lithography and microscopy. This attribute is complemented by the unique opportunity for element specific identification presented by the large number of atomic resonances, essentially for all materials in this range of photon energies. Together, these have driven the need for new short-wavelength radiation sources (e.g. third generation synchrotron radiation facilities), and novel optical components, that in turn permit new research in areas that have not yet been fully explored. This dissertation is directed towards advancing this new field by contributing to the characterization of spatial coherence properties of undulator radiation and, for the first time, introducing Fourier optical elements to this short-wavelength spectral region.

The first experiment in this dissertation uses the Thompson-Wolf two-pinhole method to characterize the spatial coherence properties of the undulator radiation at Beamline 12 of the Advanced Light Source. High spatial coherence EUV radiation is demonstrated with appropriate spatial filtering. The effects of small vertical source size and beamline apertures are observed. The difference in the measured horizontal and vertical coherence profile evokes further theoretical studies on coherence propagation of an EUV undulator beamline. A numerical simulation based on the Huygens-Fresnel principle is performed. 
Accurate knowledge of the refractive index in this wavelength region is of fundamental importance for the design of optical systems. However, due to the high absorption, no previous direct measurement of the real part of the refractive index has been performed at EUV wavelengths. To overcome these limitations, a novel diffractive optical element based on Fourier optics techniques is invented, fabricated, and demonstrated for the first time. The improved efficiency of the interferometer employing this novel optical element enables the first direct measurement of the refractive index at EUV wavelengths. Both the real and imaginary parts of the complex refractive indices are measured directly, without recourse to Kramers-Kronig transformations. Data for $\mathrm{Al}$ and $\mathrm{Ni}$, in the vicinity of their $\mathrm{L}$ and M-edges, respectively, are presented as first examples of this technique.

The first novel Fourier optical element used in the above EUV interferometer is also discussed in detail. This diffractive optical element, when illuminated by a uniform plane wave, will produce two symmetric off-axis first order foci suitable for interferometric experiments. In addition to the symmetricalness, the flux throughput is improved by $\sim 10$ times as compared with separate elements providing the same functionality. The efficiency of this optical element is measured. Future work on computer generated holograms is suggested and compared with the Fourier optical element. The invention of this Fourier optical element opens a new era in the use of sophisticated optical techniques at short wavelengths.

Professor David Attwood Dissertation Committee Chair 
To the loving memory of my Father, who loved me more than himself. 


\section{Contents}

List of Figures vii

1 Introduction $\quad 1$

1.1 Motivation . . . . . . . . . . . . . . . . . 1

1.2 EUV wavelength: opportunities and challenges . . . . . . . . . 2

1.3 Coherence . . . . . . . . . . . . . . . . . . . 3

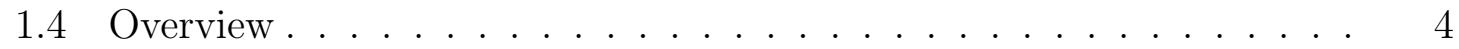

2 Spatial coherence characterization of undulator radiation $\quad 7$

2.1 Introduction . . . . . . . . . . . . . . . . 7

2.2 Mathematical description . . . . . . . . . . . . . . . . . . 10

2.3 Experiment . . . . . . . . . . . . . . . . . . 12

2.3.1 Effect of monochromator exit-slit . . . . . . . . . . . . 22

2.3.2 Wave-front null test . . . . . . . . . . . . . . . . 23

2.4 Conclusion . . . . . . . . . . . . . . . . . . . . . . . . 24

3 Analysis of the illumination coherence properties of systems where the Van Cittert-Zernike theorem does not apply 26

3.1 Introduction . . . . . . . . . . . . . . . . . 26

3.2 Rigorous mutual coherence propagation for undulator beamlines . . . 28

3.2.1 Undulator radiation as an incoherent source . . . . . . . . . 28

3.2.2 Zernike approximation for a condenser system . . . . . . . . 28

3.2.3 Undulator beamline as a condenser: an example . . . . . . . . 29

3.3 Object-image coherence relation . . . . . . . . . . . . . . . 31

3.3.1 Zernike approximation . . . . . . . . . . . . . 33

3.4 Point spread functions for aberrated pupils . . . . . . . . . . . . 35

3.4.1 Distortion \& Defocus . . . . . . . . . . . . . . . 35

3.4 .2 Astigmatism . . . . . . . . . . . . . . . . . . . . . . . . . 42

3.4 .3 Coma .......................... 43

3.5 Numerical evaluation results . . . . . . . . . . . . . . . . . . 44

3.6 Conclusion . . . . . . . . . . . . . . . . . . . 50 
4 Diffractive optical elements based on Fourier optical techniques: A new class of short wavelength optical elements $\quad 51$

4.1 Introduction . . . . . . . . . . . . . . . . . 51

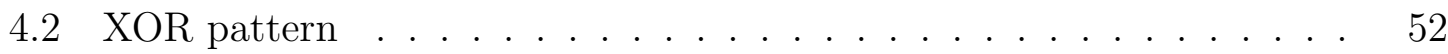

4.2.1 Simulation of the XOR pattern . . . . . . . . 56

4.3 Efficiency of the XOR pattern . . . . . . . . . . . . . 57

4.4 Visible light experiment . . . . . . . . . . . . . . . . . 58

4.5 First use in EUV interferometry . . . . . . . . . . . . . . . 61

4.6 Comparison to the computer generated hologram CGH . . . . . . . . 65

4.7 Conclusion . . . . . . . . . . . . . . . . . . . 68

5 Direct index of refraction measurements at EUV wavelengths with $\begin{array}{lr}\text { a novel interferometer } & 69\end{array}$

5.1 Introduction . . . . . . . . . . . . . . . . . . . . 69

5.2 Interferometry . . . . . . . . . . . . . . . . . . 71

5.2 .1 Principle . . . . . . . . . . . . . . . . . 71

5.2 .2 The interferometer . . . . . . . . . . . . . 73

5.2.3 Novel diffractive optical element . . . . . . . . . . . . . . . 73

5.2.4 Analysis method . . . . . . . . . . . . . . 76

5.3 Experimental setup . . . . . . . . . . . . . . . . . . . . . . 79

5.4 Experimental results . . . . . . . . . . . . . . . . . . . . . . . . . . . . . . . . . 80

5.4 .1 Aluminum across its L-edge . . . . . . . . . . . . . 80

5.4 .2 Nickel across its M-edge . . . . . . . . . . . . . . . 81

5.5 Conclusion . . . . . . . . . . . . . . . . . . . 81

6 Conclusion $\quad 84$

A Free space coherence propagation of an incoherent source with Gaussian$\begin{array}{lr}\text { shaped intensity distribution } & 87\end{array}$

A.1 Fourier transform of a Gaussian distribution function . . . . . . . . . 87

A.2 Coherence distribution after propagating from the undulator exit-plane 88

A.2.1 Van Cittert-Zernike Theorem . . . . . . . . . . . . 88

A.2.2 Incoherent source with Gaussian intensity distribution . . . . 89

$\begin{array}{ll}\text { B Pupil function of a thin lens } & 91\end{array}$

C Holographic interferometry - Retrieving phase information from an $\begin{array}{lr}\text { intensity detector } & 92\end{array}$

C.1 Mathematical preliminaries .................. . . 92

C.2 Holographic interferometry . . . . . . . . . . . . . . . . . . . . 94

C.2.1 Far-field coherent field propagation . . . . . . . . . . . . 94

C.2.2 From intensity distribution to auto-correlation . . . . . . . . . 94

C.2.3 Aperture geometry . . . . . . . . . . . . . . . . 95 
$\begin{array}{ll}\text { D Phase grating } & 97\end{array}$

D.1 $\pi$-phase-shift grating . . . . . . . . . . . . . . . . . 97

D.1.1 Fourier series expansion . . . . . . . . . . . . . . . . 98

D.2 Efficiency of $\pi$-phase-shift grating . . . . . . . . . . . . . . . . . . . . 99

D.2.1 Efficiency of a 50\% duty-cycle $\pi$-phase-shift grating . . . . . . 100

$\begin{array}{ll}\text { Bibliography } & 102\end{array}$ 


\section{List of Figures}

2.1 Young's two-pinhole interferometer for spatial coherence measurement. 10

2.2 Beamline 12 diagram. . . . . . . . . . . . . . . . . . . . . 13

2.3 Measured two-pinhole interference patterns for horizontal pinhole separations of 3, 4, 6 and 9- $\mu \mathrm{m}$, for a wavelength of $13.4 \mathrm{~nm}$ and a beamline acceptance half-angle of $48 \mu \mathrm{rad}$. The pinhole diffraction patterns overlap and produce an interference pattern within the Airy envelope. The interference patterns are recorded on an EUV sensitive CCD camera, located $26 \mathrm{~cm}$ downstream of the pinhole mask. Pinhole diameter range from 400 to $500 \mathrm{~nm}$, but are equal in their respective pairs. As shown in the lineouts, fringe visibility of the modulation decreases for larger separations. Spectral resolution for these measurements is 55. .

2.4 Measured two-pinhole interference patterns for vertical pinhole separations of 1 and $6-\mu \mathrm{m}$, at a wavelength of $13.4 \mathrm{~nm}$, and an acceptance half-angle of $48 \mu \mathrm{rad} . \ldots \ldots \ldots \ldots$

2.5 The measured coherence factor $\left|\boldsymbol{\mu}_{12}\right|$ as function of pinhole separation distance for (a), horizontally and (b), vertically separated pinholes. For these measurements, a $48 \mu \mathrm{rad}$ half-angle acceptance aperture is used. The measured spatial coherence distance decreases with larger pinhole separations as expected. Larger uncertainty in the vertical $3 \mu \mathrm{m}$ separation may be due to a smaller number of interferograms collected in this case. The data points are fitted to a Gaussian curve in each case. 19

2.6 Comparison of measured and simulated results. . . . . . . . . . . . . 21

2.7 Effect of the monochromator exit-slit. . . . . . . . . . . . . . 22

2.8 Quality of the pinhole generated wave-front. . . . . . . . . . . . . 24

3.1 Coordinate system . . . . . . . . . . . . . . . . . . . . . 31

3.2 Procedure for the calculation of defocus/distortion PSF. . . . . . . . 41 
3.3 Simulation results for a large $(1.6 \mathrm{~mm} \times 1.6 \mathrm{~mm})$ uniform source. Intensity (left column) and coherence (right column) distributions resulting from the various pupil aberrations (a defocus, $\mathbf{b}$ astigmatism, c coma). The coherence distributions are all essentially Airy patterns as predicted by the Zernike approximation. . . . . . . . . . . .

3.4 Simulation results: Intensity and coherence distribution at the condenser image-plane resulting from the un-aberrated condenser pupil and the Gaussian-shaped incoherent source of $\left(\sigma_{x}, \sigma_{y}\right)=(260 \mu m, 16 \mu m) .46$

3.5 Simulation results that show the effect of aberrations for the Gaussianshaped incoherent source: Intensity (left column) and coherence (right column) distribution at resulting from the various aberrations (a defocus, b astigmatism, c coma). . . . . . . . . . . . . . . .

3.6 Simulation results using a point source (coherent source): Intensity (left column) and coherence (right column) distributions resulting from the various pupil aberrations (a defocus, $\mathbf{b}$ astigmatism, $\mathbf{c}$ coma). The coherence distribution is a constant 1 , which shows that the field is fully coherent as expected. . . . . . . . . . . . . .

4.1 Bitwise XOR operation: The binary grating and zoneplate are first pixelized, with each pixel being either 1 or 0 for transmission and absorption, respectively. The two pixelized patterns are then overlapped and compared pixel by pixel to produce the resulting XOR pattern, i.e. at each pixel position, if the pixel values of the grating and zoneplate are the same (both 0's or both 1's), the value of the corresponding pixel on the XOR pattern is 0 . Otherwise, the value of the corresponding pixel on the XOR pattern is $1 \ldots \ldots \ldots$. . . . . . . .

4.2 Computer simulation of the XOR pattern: The parameters used in this simulation are set equal to the actual fabricated element. The pattern in (a) is obtained by taking the "exclusive or (XOR)" of the binary grating and zone-plate. $4096 \times 4096$ pixels are used to generated this pattern. This pattern is then Fresnel-propagated in computer by one focal length and the resulting intensity distribution is shown in (b). A horizontal cross-section through the focal spots is also shown. The two symmetric off-axis first order foci is clearly visible in this simulation. The other two outer spots are caused by the third orders $(m= \pm 3)$ of the grating, with 9 times lower intensity. . . . . . . . . . 
4.3 A visible light experiment is performed in order to directly verify the intensity distribution at the back focal plane of the XOR pattern. For comparison, an OR pattern obtained by taking the bit-wise OR of a grating and a zoneplate is also fabricated. The effect of this OR pattern is equivalent to that of a grating and a zoneplate placed in tandem, which is the conventional setup for interferometric experiments. Part (a) shows that the intensity distribution at the back focal plane of the XOR pattern consists of only two symmetric off-axis foci, as predicted by the theory. As a comparison, the focal plane intensity distribution of the OR pattern is shown in (b), which has three foci, with one strongest on-axis focus and two weaker off-axis symmetric foci. The grating used by the XOR and OR patterns in this visible experiment has a period of $5 \mu \mathrm{m}$ and the diameter and the outermost zone-width of the zoneplate is $D=5 \mathrm{~mm}$ and $2 \mu \mathrm{m}$, respectively. A He-Ne laser $(\lambda=633 \mathrm{~nm})$ is used for illuminating the XOR and OR patterns. . .

4.4 A microscope image of the OR pattern used in the experiment with visible light. The grating period is $5 \mu \mathrm{m}$. The zoneplate diameter is $5 \mathrm{~mm}$ and the outermost zone-width is $2 \mu \mathrm{m}$. . . . . . . . . . .

4.5 The center part of the XOR pattern is shown. This diffractive optical element is obtained by taking the bit-wise XOR of a binary amplitude grating and a binary amplitude zoneplate. The functionality of this XOR pattern is equivalent to that of a binary phase grating overlapping a binary amplitude zoneplate, as discussed in the text. The grating used here has a $16 \mu \mathrm{m}$ period ( $8 \mu \mathrm{m}$ line-space) and the zoneplate has a $400 \mu m$ diameter and a $0.2 \mu m$ outermost zone-width. . . . . . . . .

4.6 The edge of the XOR pattern is shown here. The outmost zone width is seen to be $0.2 \mu \mathrm{m}$ and the alternation of opaque and transparent zones over the grating half-period is also shown. . . . . . . . . . .

4.7 The efficiency of this XOR pattern is measured by scanning a knifelike beam-stop across the focal plane. Starting with the beam-stop placed at the back focal plane such that the entire beam is blocked, as the beam-stop slowly moves aside, the total counts on the CCD increases, allowing fractions of light to pass. The constant slope of the two straight sections results from the effect of zeroth order (straight through) light. The two abrupt steps at the center is caused by the two symmetric off-axis first order foci being released one at a time by the beam-stop. Their strength is shown to be around $4.0 \%$, which agrees with the theoretical value. . . . . . . . . . . . . . . . 
4.8 In part (a), the object wave (in red) which consists of two converging spherical wavefronts interferes with a reference plane wave (in blue) and the resulting intensity interference pattern, which is usually referred to as Computer Generated Hologram, is shown in (b). This CGH is then binarized for nanofabrication by e-beam lithography. Part (c) shows its binarized form. When illuminated by a uniform plane wave, this optical element reconstructs the object wave (two converging spherical waves) as shown in (d). Note that the two spots are symmetrically off-axis. . . . . . . . . . . . . . . . . . .

5.1 Experimental Setup: A $5 \mu \mathrm{m}$ pinhole is place at the focus of the beamlines optics to provide spatially coherent illumination for this interferometer. The grating serves as the beam splitter which effectively creates orders of virtual sources out of the pinhole. The zoneplate then images the pinhole, together with all the virtual sources created by the grating, to the plane of the sample mask. This mask consists of two $5 \mu \mathrm{m} \times 5 \mu \mathrm{m}$ windows allowing only the two symmetric orders $(m, n)=( \pm 1,1)$ to pass. The test material is then shuffled in and out over one of the windows and an back-thinned EUV-sensitive CCD camera records the respective interferograms for comparison. The functions of the grating and zoneplate have been combined into a single diffractive element following Fourier optical techniques. . . . . . . . . . .

5.2 Fourier method for phase shift $\Delta \phi$ determination. . . . . . . . . . . 77

5.3 Fourier method for determining visibility. . . . . . . . . . . . . 79

5.4 The experimental results are shown in blue. It is evident that the aluminum L-edge is resolved both in $\delta$ and $\beta$ where the position of the $L_{2}$ and $L_{3}$ edges are $73.1 \mathrm{eV}$ and $72.7 \mathrm{eV}$, respectively. Note that the values of $\delta$ and $\beta$ are obtained directly (independently) from phase shift and visibility change, respectively. The $\delta$ and $\beta$ values from Palik (in red) is derived from Kramers-Kronig transformation of a compilation of absorption and reflectance data. The two data sets agree fairly well both in $\delta$ and $\beta \ldots \ldots \ldots \ldots \ldots . \ldots \ldots$

5.5 Nickel: The experimental results are shown in blue. The $M_{3}$ edge at $66.2 \mathrm{eV}$ is clearly resolved and the $\delta$ and $\beta$ values at this edge are seen to be closely correlated. The typical exposure time $(\sim 200 \mathrm{sec})$ for the nickel interferograms is approximately 10 times longer than that of aluminum due to lower beamline flux at low energy. The stages in the experimental setup drift over longer exposure time, thus causing larger error bars in the nickel data. For comparison, current standard nickel refractive indices are shown in red. . . . . . . . . . . . .

D.1 (a) $\pi$-phase-shift grating. (b)Binary amplitude grating. . . . . . . . 98 


\section{Acknowledgements}

First and foremost, I would like to express my deepest gratitude and appreciation to my thesis advisor, Prof. David Attwood. It is his faith in me that starts and sustains this dissertation work. The guidance he provided at every step of my graduate career has kept my research on track. Most importantly, the tremendous effort he exerted in helping me overcome my weakness has made me a different person.

I would like to express my most sincere gratitude and appreciation to Patrick Naulleau, who has been my mentor in every way in the past five years. He has spared no time in explaining every subtleties of coherence and taught me everything I know today about optical coherence. His encouragement and faith has sustained me through the worst time of my graduate career. His perspective about life, and about the meaning of everything I struggled for, has kept me afloat in all these years.

I would like to thank Prof. Jack Welch and Prof. Raymond Chiao for kindly serving on my dissertation committee.

It is the support, understanding, and encouragement, given to me by my loving wife, Shiang-Ling, that makes this dissertation possible. I would also like to thank my Mother, for her strength sustains the entire family.

I would like to thank Erik Anderson for numerous enlightening and useful conversations, and for providing the world's best quality nano-structures. I would also like to thank Ken Goldberg and Eric Gullikson for various exciting and useful conversations. Brian Hoef and Paul Denham have provided tremendous help on my vacuum chamber. I would also like to thank Phil Batson and his engineering team, Kevin Bradley, Seno Rekawa, and Gideon Jones for excellent mechanical works; Bruce Harteneck and Deirdre Olynick for their helps in the nano-lab; Jeff Gamsby, Ron Tackaberry, and Jos Polman for constant support of my Linux box. I would like to express a special thank to Linda Geniesse, whose excellent artworks tremendously enhance this dissertation. Last but definitely not least, I would like to thank future Doctors Kris Rosfjord and Michael Shumway for their fellowship (and cookies) out at Beamline 12. 


\section{Chapter 1}

\section{Introduction}

\subsection{Motivation}

Coherent radiation offers important opportunities for both science and technology. The well defined phase relationships characteristic of coherent radiation, allow for diffraction-limited focusing (as in scanning microscopy), set angular limits on diffraction (as in protein crystallography), and enable the convenient recording of interference patterns (as in interferometry and holography). While coherent radiation has been readily available and widely utilized at visible wavelengths for many years [1-7], it is just becoming available for wide use at shorter wavelengths [8-11]. This is of great interest as the shorter wavelengths, from the extreme ultraviolet (EUV: 10-20 nm wavelength), soft x-ray (SXR: 1-10 nm), and x-ray ( $<1 \mathrm{~nm}$ ) regions of the spectrum, correspond to photon energies that are well matched to the primary electronic resonances (K-shell, L-shell, etc.) of essentially all elements, thus providing a powerful combination of techniques for the elemental and chemical analysis of physical and biological materials at very high spatial resolution. Tunable, coherent radiation in these spectral regions is available primarily due to the advent of undulator radiation at modern synchrotron facilities [12-18], where relativistic electron beams of small cross-section transverse periodic magnet structures, radiating very bright, powerful, and spatially coherent radiation at short wavelengths. The development of optical elements follow as short wavelength radiation sources are made available. 
Due to the large absorption at these short wavelengths, devising optical elements that serve the specific needs of an application is of crucial importance. This dissertation is directed towards advancing this new field by contributing to the characterization of spatial coherence properties of undulator radiation and, for the first time, introducing Fourier optical elements to this short-wavelength spectral region.

\subsection{EUV wavelength: opportunities and challenges}

With proper optics, shorter wavelength results in better spatial resolution for imaging systems. Lithographic tools (steppers) used by the semiconductor industry to print nanometer transistor patterns are constantly evolving toward shorter wavelengths in order to reduce the circuit dimension. High power EUV radiation produced by xenon laser plasma sources and multi-layer reflective mirrors working specifically at $92.5 \mathrm{eV}(\lambda=13.4 \mathrm{~nm})$ provide the crucial optical flux throughput that makes EUV Lithography the leading next generation lithographic technology for feature sizes of $45 \mathrm{~nm}$ and smaller. In seeing smaller features, soft x-ray microscopes have been an increasingly important tool in both biological [19,20] and magnetic material studies [21]. High resolution (outermost zone-width $\Delta r=25 \mathrm{~nm}$ ) zoneplates fabricated by electron-beam tools [22] deliver the required diffraction-limited wavefront for x-ray microscopic imaging. The water-window at $500 \mathrm{eV}(2.4 \mathrm{~nm})$ makes x-ray microscopes especially attractive for biologist because of its capability to see samples at a higher spatial resolution as compared with visible-light microscopes.

The various atomic resonances are the element-specific signatures of the particular materials, and the capability of probing atomic resonances at-wavelength allows accurate element-sensitive analysis that is essential in material science [23], environmental science [24], and surface science [25]. Photoelectron spectroscopy and absorption spectroscopy are the two most commonly used experimental techniques in this respect [26]. Astronomers also exploit this element-specific property by employing telescopes with narrow bandpass multilayer mirrors to detect specific emission lines of atoms/ions [27].

Concomitantly, the challenges in EUV/SXR research stem from exactly the same 
properties that provide these enormous opportunities. The large amount of atomic resonances of essentially all materials at EUV/SXR spectral region results in high absorption, thus limiting experimental techniques. For example, the use of traditional refractive lenses is prohibited due to the large absorption at these wavelengths. This obstacle of high absorption has been continuously attacked from both fronts: seeking EUV/SXR radiation sources of higher power, and creating optical elements that can more effectively harness the short wavelength radiation.

The advent of high power, high brightness, short wavelength radiation provided by the third generation synchrotron radiation facilities, e.g. undulators and wigglers, essentially gave birth to this new field. Continuing efforts to develop a fourth generation synchrotron radiation source, perhaps a free electron laser [28], is expected to produce a higher power, higher brightness and highly coherent x-ray source.

The development of optical elements comprises the other front of the exertion in short wavelength optics. As mentioned above, refractive lens are ineffective at EUV and x-ray wavelengths due to high absorption. A variety of optical elements, e.g. Fresnel zone-plates, multi-layer mirrors, glancing incidence mirrors, capillary optics [29], compound refractive lenses [30], photon sieves [31], and Fourier optical elements [11], have been devised to satisfy the various wavefront shaping requirements of short wavelength experiments. In this dissertation, the first Fourier optical element, an XOR pattern which combines the functionalities of a grating and a zoneplate, is demonstrated with applications in EUV interferometry.

\subsection{Coherence}

An optimal degree of coherence is needed for a given application. For example, interference experiments such as interferometry and holography usually require a higher degree of coherence, while image formation experiments necessitate delicate control of partial coherence. Therefore, the ability to measure and control the coherence properties of an imaging system is of crucial importance for all optical experiments.

At shorter wavelengths, the importance of coherence on an imaging system has long been ignored, mainly because of the lack of sophisticated optical systems at these 
wavelengths. Advances in both source and optical technology now permit the emergence of more sophisticated short wavelength optical systems, e.g. EUV lithography, x-ray microscopy, and EUV/SXR interferometry. Therefore, an increasing demand for a better understanding of coherence at short wavelengths arises. The experimental studies of the optical coherence properties of EUV/SXR optical systems are all fairly recent. Indeed, demonstrations of the ability to accurately measure $[9,32]$ and control $[33,34]$ the degree of coherence have only been published very recently. This dissertation describes the first short-wavelength Thompson-Wolf two-pinhole characterization of the spatial coherence properties of undulator radiation and confirms the anticipated coherence at these very short wavelengths. Applications that exploit this better understanding of short-wavelength coherence are described in the following chapters.

\subsection{Overview}

Chapter 2 starts with a spatial coherence characterization of the EUV undulator radiation at the Advanced Light Source (ALS) in Berkeley, a third generation synchrotron facility [35]. This first conditioning experiment uses the Thompson-Wolf two-pinhole method to characterize the spatial coherence properties of the undulator radiation at Beamline 12 of the Advanced Light Source. The effects of elliptical source size and beamline apertures are observed. The result of this spatial coherence characterization determines the optimal size of the spatial filter (i.e. pinhole) used for EUV interferometry described in Chapter 5. This optimized pinhole provides just enough spatial filtering without excessively sacrificing optical flux, so that it provides the required spatial coherence necessary for the interferometric experiment and at the same time allows an optimal amount of flux through it.

Chapter 3 describes the effect of aberrations on the spatial coherence properties of an undulator beamline, which is recognized as a critically-illuminated system. The commonly used Zernike approximation [6], which states that aberrations have no effect on the spatial coherence distribution at the image-plane of the condenser, is no longer valid in the case of undulator radiation source due to the exceedingly small 
vertical source size. Therefore, a numerical simulation based on the Huygens-Fresnel principle is required to describe the spatial coherence properties of this criticallyilluminated imaging system. The simulated spatial coherence distribution is shown to be affected by the various aberrations, as observed in the experimental results presented in Chapter 2.

Chapter 4 is devoted to the first novel Fourier optical element, the XOR pattern, that optimizes the EUV interferometer described in Chapter 5 by providing important new advances in the properties of optical flux throughput and symmetricalness. This diffractive element, based on Fourier optical techniques, for use in EUV/SXR experiments, has been fabricated and demonstrated. This diffractive optical element, when illuminated by a uniform plane wave, will produce two symmetric off-axis first order foci suitable for interferometric experiments. The efficiency of this optical element and its use in direct interferometric determination of optical constants are also discussed. Its use opens a new era in the use of sophisticated optical techniques at short wavelengths.

Chapter 5 describes the first direct index of refraction measurement at EUV wavelengths with a novel interferometer. Accurate knowledge of the refractive index in this wavelength region is of fundamental importance for the design of optical systems. However, due to the high absorption, no previous direct measurement of the real part of the refractive index has been performed at EUV wavelengths. To overcome these limitations, a novel diffractive optical element, based on Fourier optics techniques, for use in EUV/soft x-ray interferometric experiments is invented, fabricated and demonstrated for the first time. The efficiency of the interferometer employing this novel optical element improves by $\sim 10$ times, compared with a separate grating and zoneplate setup, thus enabling the first direct measurement of the refractive index at EUV wavelengths. Both the real and imaginary parts of the complex refractive indices are measured directly by this technique without recourse to Kramers-Kronig transformations [36]. Data for $\mathrm{Al}$ and $\mathrm{Ni}$, in the vicinity of their L and M-edges, respectively, are presented as first examples of this technique. Undulator radiation available at the third generation synchrotron facilities (in this case the Advanced Light Source) provides the high brightness, high coherence short wavelength radiation necessary for 
this interferometric experiment.

The contribution of this dissertation to the field of short wavelength optics is again two-fold: characterizing the spatial coherence properties of the new radiation source and, for the first time, introducing Fourier optical techniques to short wavelength optics. 


\section{Chapter 2}

\section{Spatial coherence characterization of undulator radiation}

The coherence properties of undulator radiation at extreme ultraviolet (EUV) wavelengths are measured using the Thompson-Wolf twopinhole method. The effects of elliptical source size and beamline apertures are observed. High spatial coherence EUV radiation is demonstrated. Projection of these same capabilities to the x-ray region is straightforward.

\section{$2.1 \quad$ Introduction}

Coherent radiation offers important opportunities for both science and technology. The well defined phase relationships characteristic of coherent radiation, allow for diffraction-limited focusing (as in scanning microscopy), set angular limits on diffraction (as in protein crystallography), and enable the convenient recording of interference patterns (as in interferometry and holography). While coherent radiation has been readily available and widely utilized at visible wavelengths for many years $[1,3-5,7]$, it is just becoming available for wide use at shorter wavelengths $[8,9]$. This is of great interest as the shorter wavelengths, from the EUV (10-20 nm wavelength), soft x-ray (1-10 nm), and x-ray ( $<1 \mathrm{~nm}$ ) regions of the spectrum, correspond to photon energies that are well matched to the primary electronic resonances (Kshell, L-shell, etc.) of essentially all elements, thus providing a powerful combination 
of techniques for the elemental and chemical analysis of physical and biological materials at very high spatial resolution. Tunable, coherent radiation in these spectral regions is available primarily due to the advent of undulator radiation at modern synchrotron facilities [12-18], where relativistic electron beams of small cross-section transverse periodic magnet structures, radiating very bright, powerful, and spatially coherent radiation at short wavelengths. Recent progress with EUV lasers [37,38], high laser harmonics [39,40], and free electron lasers [28] may soon add to these capabilities. In this chapter, the classic two-pinhole diffraction technique [5], an extension of Young's two-slit interference experiment [7], is utilized to simply and accurately characterize the degree of spatial coherence provided by undulator radiation. It is shown that, with the aid of modest pinhole spatial filtering, undulator radiation can provide tunable short wavelength radiation with a very high degree of spatial coherence at presently available user facilities. Spatially coherent power of order $30 \mathrm{~mW}$ is available in the EUV [35], and is expected to scale with wavelength to about $0.3 \mathrm{~mW}$ in the hard x-ray region [8].

For radiation with a high degree of coherence and a well-defined propagation direction, it is convenient to describe coherence properties in longitudinal and transverse directions. For a source of diameter $d$, emission half-angle $\theta$, and full spectral bandwidth $\Delta \lambda$ at wavelength $\lambda$, relationships for full spatial coherence and longitudinal coherence length, $l_{c o h}$, are given respectively by

$$
d \cdot \theta=\lambda / 2 \pi
$$

and

$$
l_{c o h}=\lambda^{2} / 2 \Delta \lambda
$$

where $d, \theta$, and $\Delta \lambda$ are $1 / \sqrt{e}$ measures of Gaussian distributions. Based on measures of the source size and theoretical predictions of the emission angle, it is estimated that undulator radiation, as discussed in this chapter, emanating from an electron beam of highly elliptical cross-section, will approach full spatial coherence Eq. (2.1) in the vertical plane, while being coherent over only a fraction of the radiated beam in the horizontal direction. Here a detailed characterization of an undulator beamline optimized for operation in the EUV regime is presented. 
Undulator beamline 12.0 at Lawrence Berkeley National Laboratory's Advanced Light Source (ALS) was developed to support high-accuracy wave-front interferometry of EUV optical systems [41,42]. With an electron beam of elliptical cross-section, having a vertical size $d_{v}=2 \sigma_{v}=32 \mu \mathrm{m}$, and an emission half-angle $\theta=80 \mu \mathrm{rad}$ (the central radiation cone containing a $1 / N$ relative spectral bandwidth, where $\mathrm{N}$ is the number of magnet periods of the undulator), the product $d \cdot \theta$ is just slightly larger $(20 \%)$ than $\lambda / 2 \pi$ at the $13.4 \mathrm{~nm}$ wavelength used in these experiments. Thus one expects to see strongly correlated fields, of high spatial coherence, in the vertical plane. The horizontal beam size is considerably larger with $d_{h}=2 \sigma_{h}=520 \mu \mathrm{m}$, so that with approximately the same emission half-angle it is expected to be spatially coherent over only a fraction of the horizontal extent of the radiated beam.

The coherence characterization presented here is performed at the focus of the condenser system used to re-image the undulator source to the entrance of our experimental chamber. In the case of beamline 12, this condenser is a Kirkpatrick-Baez (KB) system [8]. Its focal plane serves as the entrance plane for various experiments, including EUV phase-shifting point diffraction interferometry [41-43]. The coherence measurement is based on an implementation, at a shorter wavelength, of the well known Thompson and Wolf two-pinhole experiment [5]. The Thompson and Wolf experiment is essentially an extension of Young's classic two-slit interference experiment [7], where in this case fringe visibility is recorded as a function of pinhole separation in order to determine the spatial coherence properties of the illuminating beam. Under the conditions that: (1) the pinholes are small enough such that the field within each pinhole can be regarded as constant, (2) the bandwidth of the illuminating beam is narrow enough that temporal coherence does not significantly affect fringe visibility, and (3) the intensity at the two sampled points are equal, the fringe visibility can be shown to be proportional to the magnitude of the complex coherence factor, $\left|\mu_{12}\right|,[1,3]$. Typical measured interference patterns are presented in Fig. 2.3, which shows interference modulation of the Airy envelope as a function of pinhole separation distance. These patterns provide a direct measure of the spatial coherence of undulator radiation as transported by the beamline optical system. 


\subsection{Mathematical description}

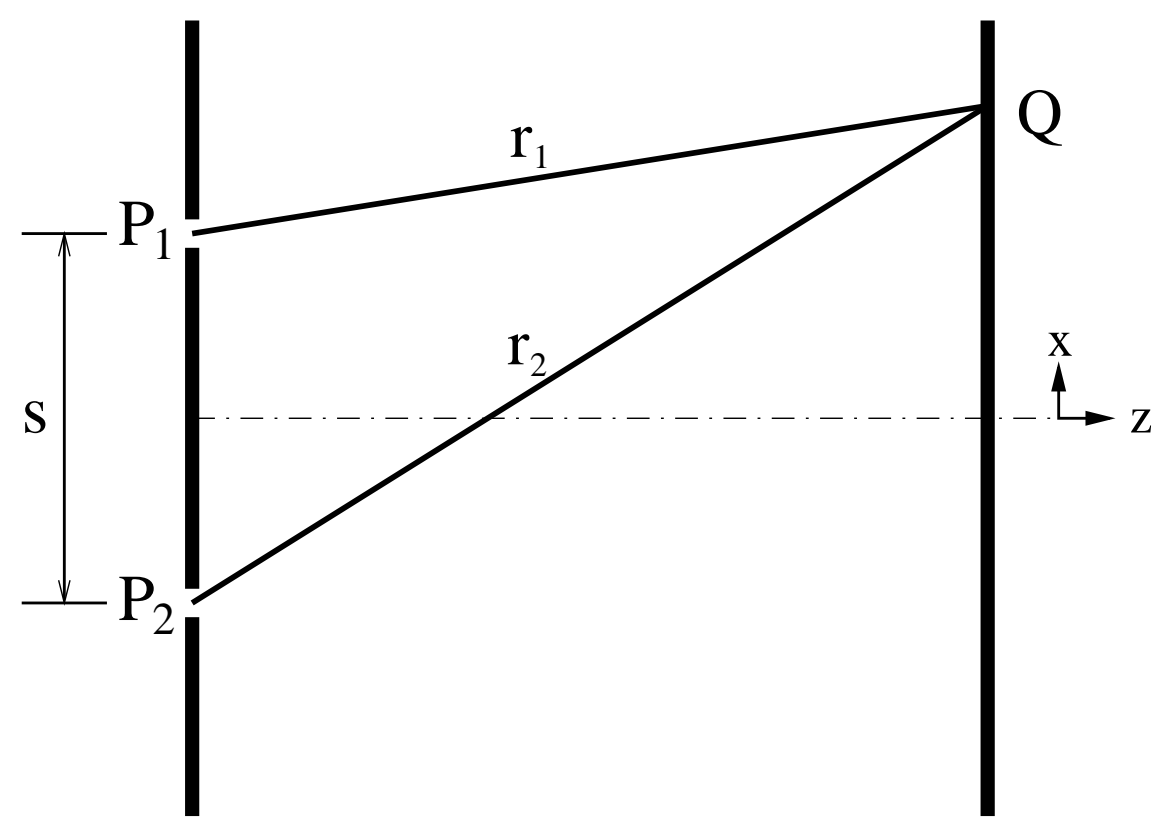

Figure 2.1: Young's two-pinhole interferometer for spatial coherence measurement.

A schematic plot of Young's experiment is shown in Fig. 2.1 where $P_{i}, r_{i}, i=1,2$ are the pinhole positions and their distance to the mixing plane, respectively. The

$a \quad r \quad \Gamma_{12}(\tau)$ which represents the cross-correlation of the light incident on pinholes $P_{1}$ and $P_{2}$ is defined by

$$
\boldsymbol{\Gamma}_{12}(\tau) \equiv\left\langle\mathbf{u}\left(P_{1}, t+\tau\right) \mathbf{u}^{*}\left(P_{2}, t\right)\right\rangle
$$

The intensity $I(Q)$ at any point $Q$ on the mixing plane is given by $I(Q)=\left\langle|\mathbf{u}(Q, t)|^{2}\right\rangle$. Assuming that the light is narrowband and the pinholes are small enough such that the analytic signal within each pinhole can be regarded as uniform, $\mathbf{u}(Q, t)$ can be represented by the weighted sum of the properly delayed analytic signals at the pinholes,

$$
\mathbf{u}(Q, t)=\mathbf{K}_{1} \mathbf{u}\left(P_{1}, t-\frac{r_{1}}{c}\right)+\mathbf{K}_{2} \mathbf{u}\left(P_{2}, t-\frac{r_{2}}{c}\right)
$$


where $\mathbf{K}_{1}, \mathbf{K}_{2}$ are purely imaginary numbers. The intensity of light at the mixing plane can therefore be represented by

$$
I(Q)=I^{(1)}(Q)+I^{(2)}(Q)+2 K_{1} K_{2} \Re e\left\{\Gamma_{12}\left(\frac{r_{2}-r_{1}}{c}\right)\right\}
$$

where $I^{(i)}(Q) \equiv\left|\mathbf{K}_{i}\right|^{2}\left\langle\left|\mathbf{u}\left(P_{i}, t\right)\right|^{2}\right\rangle, i=1,2$ is the contribution on $Q$ from the $i^{t h}$ pinhole alone and $K_{i}=\left|\mathbf{K}_{i}\right|$. To further demonstrate the rising of the fringe pattern, a normalized version of $\boldsymbol{\Gamma}_{12}(\tau)$ is defined to be

$$
\gamma_{12}(\tau) \equiv \frac{\boldsymbol{\Gamma}_{12}(\tau)}{\left[\boldsymbol{\Gamma}_{11}(0) \boldsymbol{\Gamma}_{22}(0)\right]^{1 / 2}}
$$

Equation (2.5) can then written as

$$
I(Q)=I^{(1)}(Q)+I^{(2)}(Q)+2 \sqrt{I^{(1)}(Q) I^{(2)}(Q)} \Re e\left\{\gamma_{12}\left(\frac{r_{2}-r_{1}}{c}\right)\right\}
$$

The last step in visualizing the fringe pattern is the following: since the light is assumed to be narrowband with center frequency $\bar{\nu}(\bar{\lambda}=c / \bar{\nu})$, the complex degree of coherence may be re-written in the following form

$$
\gamma_{12}(\tau)=\left|\gamma_{12}(\tau)\right| \exp \left\{-j\left[2 \pi \bar{\nu} \tau-\alpha_{12}(\tau)\right]\right\}
$$

and Eq. (2.7) would become

$$
\begin{aligned}
I(Q)= & I^{(1)}(Q)+I^{(2)}(Q)+ \\
& 2 \sqrt{I^{(1)}(Q) I^{(2)}(Q)}\left|\gamma_{12}\left(\frac{r_{2}-r_{1}}{c}\right)\right| \cos \left[2 \pi \bar{\nu} \frac{r_{2}-r_{1}}{c}-\alpha_{12}\left(\frac{r_{2}-r_{1}}{c}\right)\right]
\end{aligned}
$$

As can be seen from the above Eq. (2.9), in the vicinity of zero path length difference $\left(r_{2}-r_{1}\right) \simeq 0$, the macroscopic fringe visibility $\mathcal{V}$ is related to the microscopic complex degree of coherence $\gamma_{12}(0)$ by

$$
\mathcal{V}=\frac{2 \sqrt{I^{(1)}(Q) I^{(2)}(Q)}}{I^{(1)}(Q)+I^{(2)}(Q)}\left|\gamma_{12}(0)\right|
$$


Quasi-monochromatic conditions In some circumstances the bandwidth of the light is so narrow that the effect of temporal coherence on the fringe visibility is negligible over the observable region. If this quasi-monochromatic condition is satisfied, $\gamma_{12}(\tau)$ can be approximated by

$$
\gamma_{12}(\tau) \cong \underbrace{\gamma_{12}(0)}_{\boldsymbol{\mu}_{12}} e^{-j 2 \pi \bar{\nu} \tau}
$$

where $\boldsymbol{\mu}_{12} \equiv \boldsymbol{\gamma}_{12}(0)=\left|\boldsymbol{\gamma}_{12}(0)\right| e^{j \alpha_{12}(0)}$ is the

$$
r a r \text {. }
$$

Together with the paraxial approximation, Eq. (2.9) can be further simplified to

$$
I(Q)=I^{(1)}(Q)+I^{(2)}(Q)+2 \sqrt{I^{(1)}(Q) I^{(2)}(Q)}\left|\boldsymbol{\mu}_{12}\right| \cos \left(\frac{2 \pi}{\lambda z} s x+\phi_{12}\right),
$$

where $x$ is the axis on $Q$ that is parallel to the pinholes, $\phi_{12}=\alpha_{12}(0)$ had we chosen the optical axis to pass through the center of the pinhole pairs, $s$ is the pinhole separation and $z$ is the distance from the pinhole plane to the mixing plane. Note that for this quasi-monochromatic case, $\lambda$ is used instead of $\bar{\lambda}$.

In this experiment, the largest pinhole separation is $s=9 \mu \mathrm{m}$, which is smaller than the pixel size $(25 \mu m)$ of the CCD. The two Airy patterns, $I^{(1)}(Q)$ and $I^{(2)}(Q)$, are then basically overlapped, i.e. $I^{(1)}(Q)=I^{(2)}(Q)$ for all points $Q$ on the mixing plane. The intensity pattern on the mixing plane (CCD) is therefore

$$
I(Q)=2 I^{(1)}(Q)\left[1+\left|\boldsymbol{\mu}_{12}\right| \cos \left(\frac{2 \pi}{\lambda z} s x+\phi_{12}\right)\right]
$$

and the fringe visibility is $\mathcal{V}=\left|\boldsymbol{\mu}_{12}\right|$ over the entire observable mixing plane.

\subsection{Experiment}

The experimental system is depicted in Fig. 2.2. The beamline [35] provides an overall 60:1 demagnified image of the source in the focal plane of the KB system. The undulator employed at this beamline has a magnet period $\left(\lambda_{u}\right)$ of $8 \mathrm{~cm}$, 55 magnet periods $(N)$, and a non-dimensional magnetic field parameter $K=2.7$. The electron beam energy is $1.9 \mathrm{GeV}$, with a corresponding relativistic Lorentz factor $\gamma=3720$. The acceptance half-angle (NA) of undulator radiation for these 


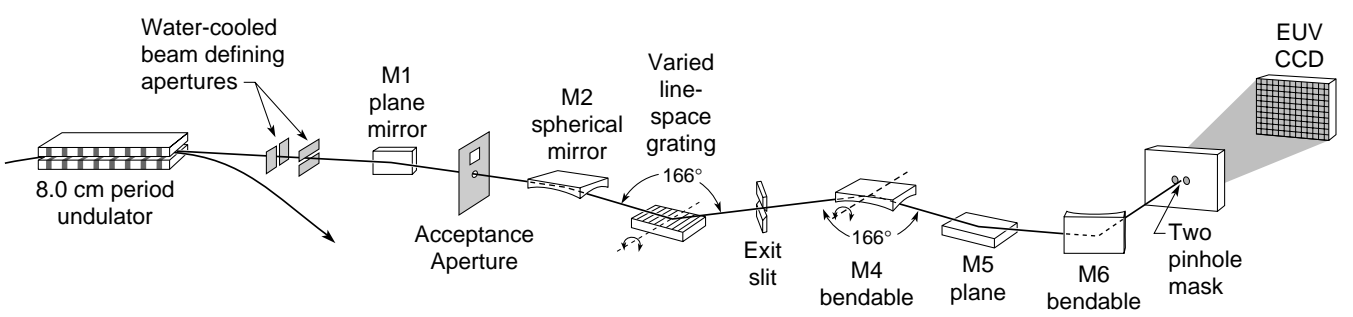

Figure 2.2: The experiment setup shows the undulator, beamline optics, monochromator grating and exit-slit, Kirkpatrick-Baez (KB) re-focusing optics (M4 and M6), and the spatial coherence measuring end-station consisting of a two-pinhole mask and an CCD electronic array detector. The two pinholes are shown here with a horizontal separation; vertical separation tests are also employed.

experiments is set by the acceptance aperture of the beamline, which is a 1.6- $\mathrm{mm}$ diameter circle placed 16.7 meters downstream of the undulator exit. This acceptance NA of $48 \mu \mathrm{rad}$ is somewhat smaller than the central radiation cone half-angle, $\theta_{c e n}=\sqrt{1+K^{2} / 2} / \gamma \sqrt{N}=80 \mu \mathrm{rad}[8,35]$. The radiation within $\theta_{c e n}$ has a natural bandwidth of $\lambda / \Delta \lambda=N$, corresponding to a longitudinal coherence length, $l_{\text {coh }}$, of $0.37 \mu \mathrm{m}$. The monochromator bandpass of this beamline can be narrowed to values as large as $\lambda / \Delta \lambda=1100$, by adjusting the size of its horizontal exit-slit. Except where stated otherwise, all experiments reported in this chapter were performed with the monochromator exit-slit set such as to pass the entire $\lambda / \Delta \lambda=55$ natural undulator bandwidth. Accounting for the 48- $\mu$ rad acceptance NA, the spatially coherent power is expected to be about $12 \mathrm{~mW}$, within a relative bandwidth of $\frac{\lambda}{\Delta \lambda}=55$ at $\lambda=13.4 \mathrm{~nm}$ [35]. Using the full 80- $\mu \mathrm{rad}$ acceptance NA defined by $\theta_{\text {cen }}$ would yield expected coherent power of $30 \mathrm{~mW}$. This bandwidth is sufficient to assure that the quasi-monochromatic condition required for this experiment is satisfied, i.e. that the temporal coherence does not significantly affect fringe visibility.

As shown in Fig. 2.2, the M2 spherical mirror images the undulator output vertically to the monochromator exit-slit. The calculated FWHM of the vertical intensity profile on the exit-slit is $17 \mu m$, neglecting aberrations on M2 mirror. The KB system is composed of two asymmetric, bendable reflective mirrors (M4 and M6). Mirror M6 directly demagnifies the undulator source in the horizontal direction by a factor of 
60, whereas M4 demagnifies the monochromator exit-slit in the vertical direction by a factor of 7.2. M2 and M4 together provide a total vertical demagnification of 60 in the plane of the two-pinhole mask.

To implement these coherence tests, a patterned mask containing multiple 450$n m$-diameter-pinhole pairs, with separations ranging from 1 to $9-\mu m$, was placed in the vicinity of the $\mathrm{KB}$ system focus, i.e. at the demagnified image of the undulator source. The 450- $\mathrm{nm}$ pinhole diameter is chosen to be significantly smaller than the expected coherence width, while providing reasonable throughput and appropriate working distance for full Airy pattern recording at the charge-coupled-device (CCD) electronic array detector. The mask, fabricated using electron-beam lithography and reactive-ion etching, consists of a 360- $n m$-thick $N i$ absorbing layer evaporated on a 100-nm-thick $S i_{3} N_{4}$ membrane. The mask features are etched completely through the membrane prior to the $N i$ evaporation, leaving the pinholes completely open in the finished mask. Pinhole circularity and size are confirmed by observing the resultant far field Airy patterns, as recorded on the CCD.

The pinhole array mask is mounted on an $x-y-z$ stage, allowing desired pinhole separations to be selected sequentially, and the coherence to be studied as a function of focal position. A back-thinned, back-illuminated, EUV sensitive CCD camera is placed $26 \mathrm{~cm}$ downstream of the mask to record the resulting interference pattern. The active area of the CCD is $25.4 \mathrm{~mm} \times 25.4 \mathrm{~mm}$, in a 1024 by 1024 pixel array. Typical exposure times for a recorded pattern vary between $50 \mathrm{msec}$ and $5 \mathrm{sec}$ depending on pinhole separation, storage ring current, and beamline apertures.

Because the divergence created by the pinhole diffraction is large relative to the pinhole separation, the two diffraction patterns overlap to a high degree on the CCD. In order to determine the magnitude of the complex coherence factor, $\left|\boldsymbol{\mu}_{12}\right|$, from the fringe visibility, one must know the relative intensities of the illuminating beam at the two pinholes or, alternatively, guarantee them to be equal. This can be challenging as the pinholes are near the KB focal plane, where the beam is small and may display structure due to aberrations in the optics. Because it is impractical to independently measure the intensity at each pinhole, we attempt to guarantee the equal intensity condition by performing a large ensemble (greater than 50) of measurements for each 
pinhole separation, intentionally displacing the pinhole pair relative to the incident beam. Because beam-intensity non-uniformity can only degrade fringe visibility, we take the highest fringe visibility from the ensemble of measurements as representing the coherence-limited fringe visibility.

Figure 2.3 shows the recorded interference patterns for horizontal pinhole separations of 3, 4, 6 and $9 \mu \mathrm{m}$. The measured magnitude of the fringe visibility decreases with larger pinhole separation as expected. Figure 2.4 shows several interference patterns obtained with vertically displaced pinhole pairs. Fringe modulation is generally better than that of horizontally displaced pinholes. In order to verify our ability to control and measure the beamline coherence properties, the measurement was repeated at a larger beamline acceptance NA. This NA can be controlled by way of the acceptance aperture described above. The measured spatial coherence decreases in both directions as expected when the $48 \mu \mathrm{rad}$ acceptance aperture is replaced by a larger aperture allowing the entire $80 \mu \mathrm{rad}$ central radiation cone to pass.

The interference pattern at the CCD is written here again from Eq. (2.13)

$$
I(x, y)=2 I^{(1)}(x, y)\left[1+\left|\boldsymbol{\mu}_{12}\right| \cos \left(\frac{2 \pi}{\lambda z} s x+\phi_{12}\right)\right]
$$

where $I^{(1)}(x, y)$ is the Airy intensity envelope in the recording plane due to pinhole diffraction, $x$ is the axis on the recording plane that is parallel to the pinhole separation, $s$ is the pinhole separation, $\lambda$ is the wavelength, and $z$ is the distance from the pinholes to the recording plane. Note that the phase $\phi_{12}$ describes the fringe shift relative to the geometric center of the interference pattern. With equi-phase illumination of the two pinholes and proper pinhole alignment, $\phi_{12}=0$. Because fringe visibility is defined as

$$
\mathcal{V} \equiv \frac{I_{\max }-I_{\min }}{I_{\max }+I_{\min }}
$$

one finds that $\mathcal{V}=\left|\boldsymbol{\mu}_{12}\right|$ as a constant over the entire interferogram for the cases considered here.

To obtain the fringe visibility from the interference pattern, we perform a twodimensional Fourier transform of the interferogram and separate the zeroth order and the two first-order peaks. As seen in Eq.(2.14), the Fourier transform of the 
(a)
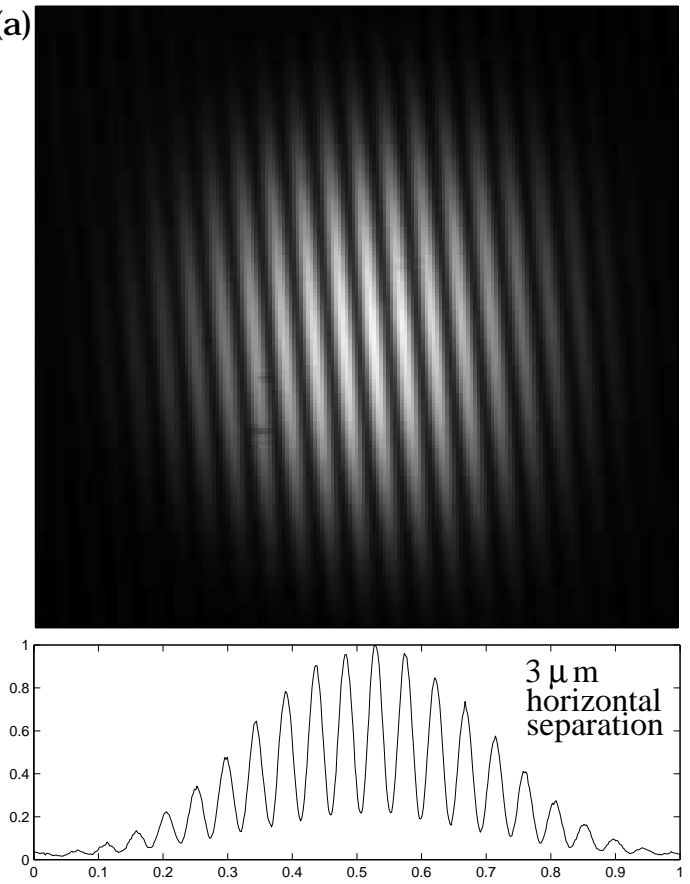

(c)
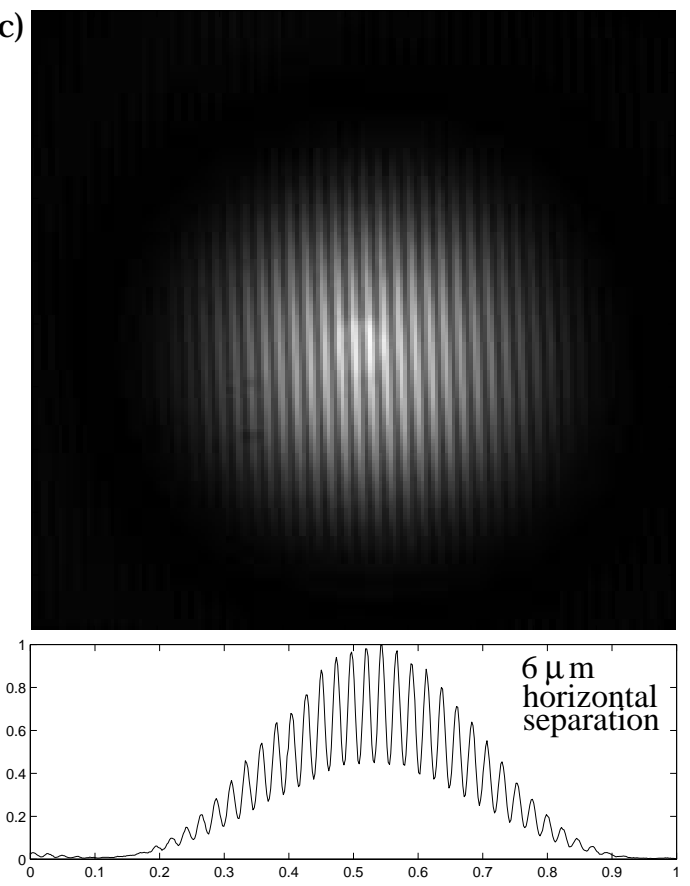

(b)
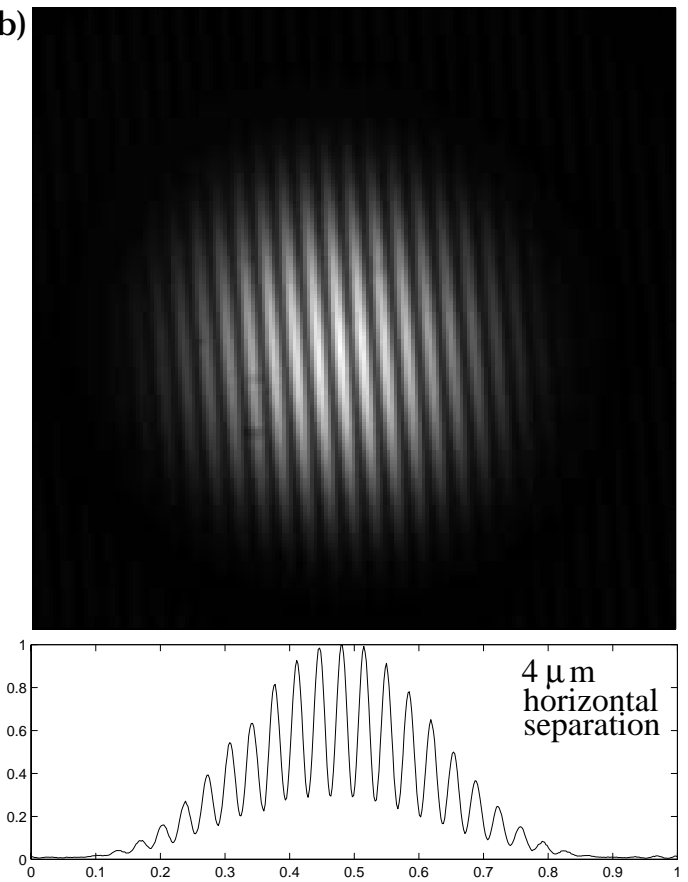

(d)
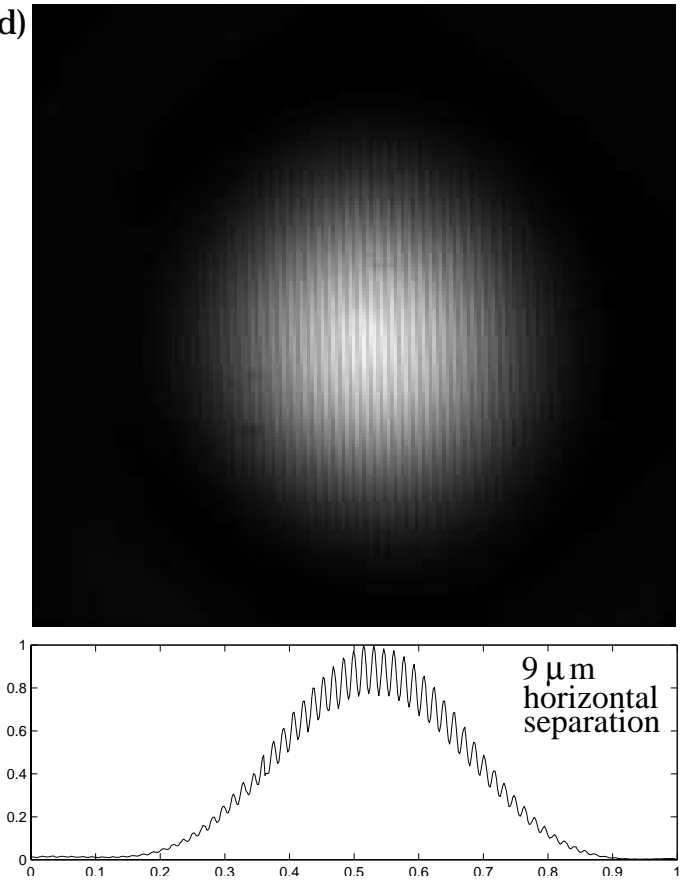

Figure 2.3: Measured two-pinhole interference patterns for horizontal pinhole separations of 3 , 4, 6 and $9-\mu \mathrm{m}$, for a wavelength of $13.4 \mathrm{~nm}$ and a beamline acceptance half-angle of $48 \mu \mathrm{rad}$. The pinhole diffraction patterns overlap and produce an interference pattern within the Airy envelope. The interference patterns are recorded on an EUV sensitive CCD camera, located $26 \mathrm{~cm}$ downstream of the pinhole mask. Pinhole diameter range from 400 to $500 \mathrm{~nm}$, but are equal in their respective pairs. As shown in the lineouts, fringe visibility of the modulation decreases for larger separations. Spectral resolution for these measurements is 55 . 
(a)
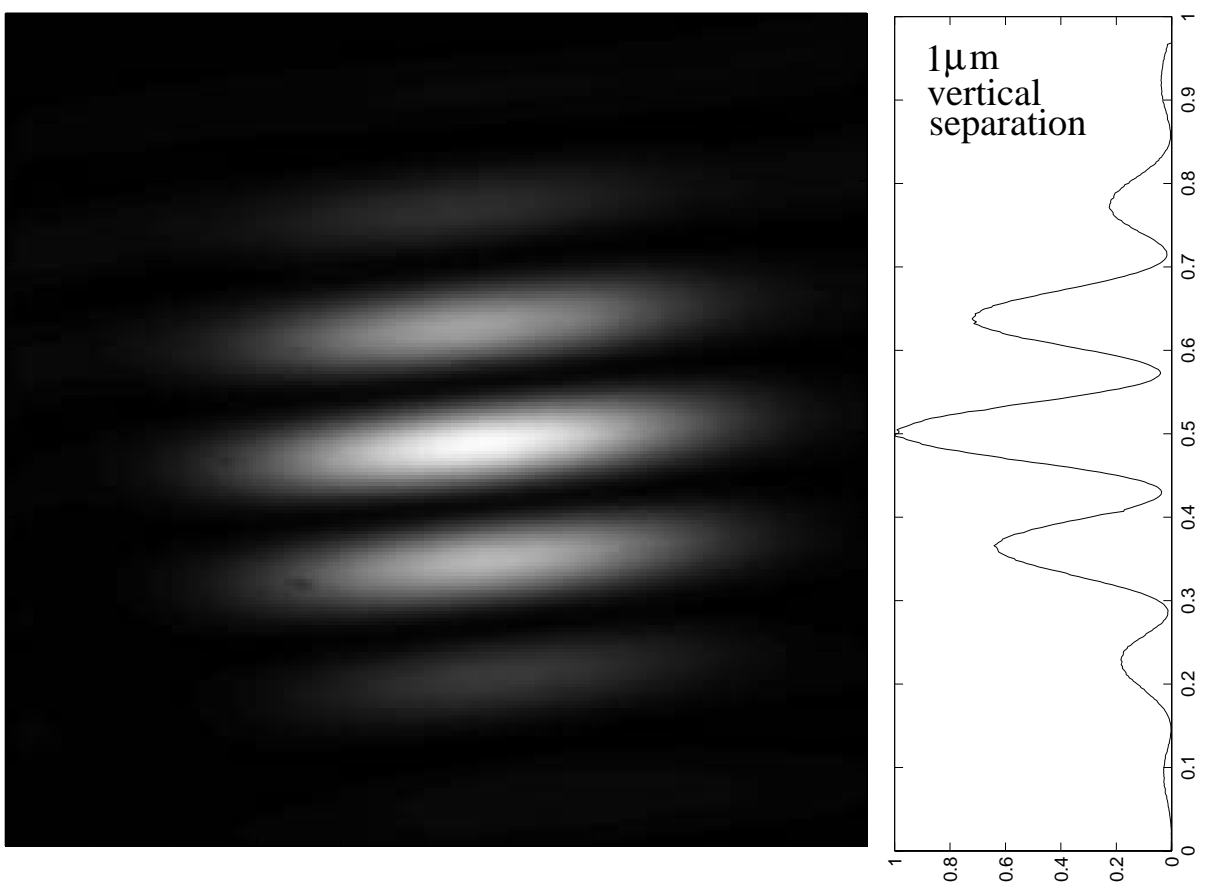

(b)
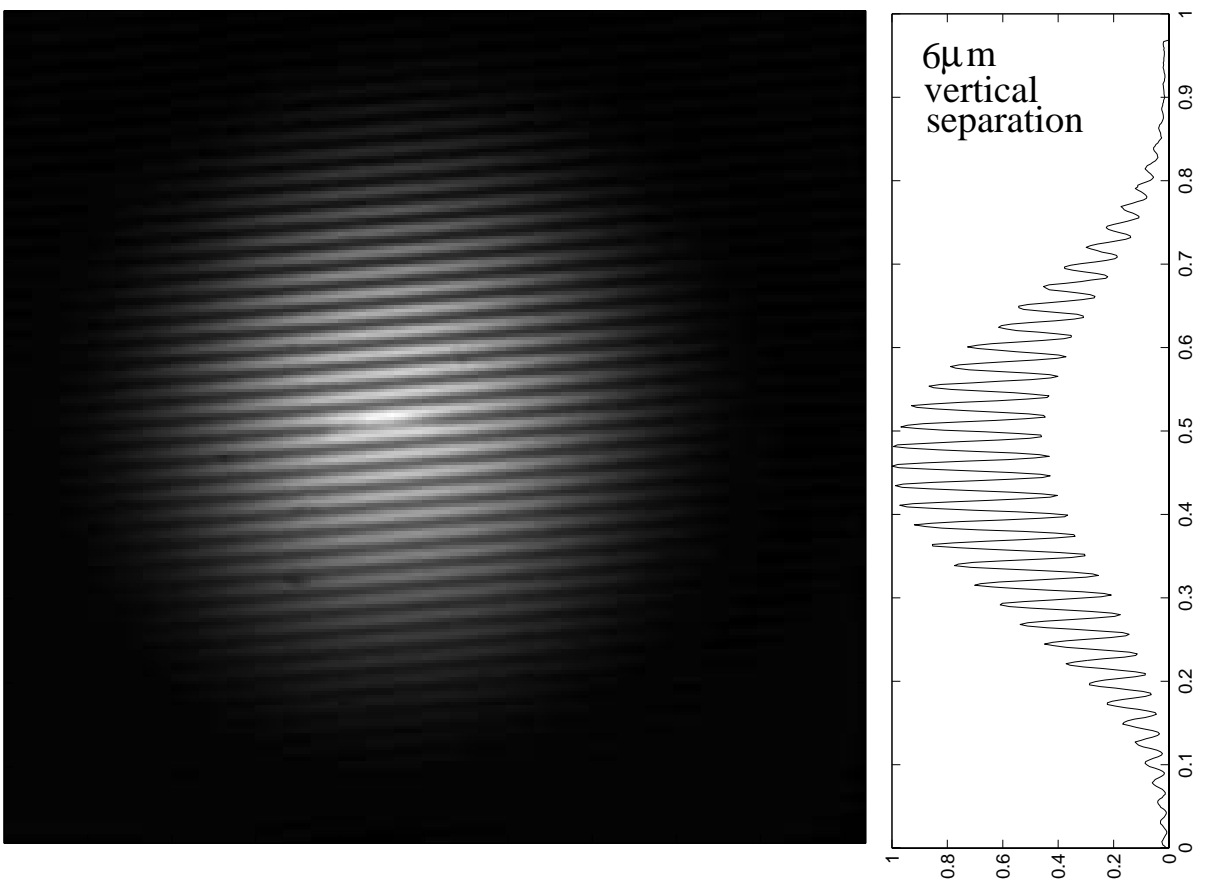

Figure 2.4: Measured two-pinhole interference patterns for vertical pinhole separations of 1 and $6-\mu \mathrm{m}$, at a wavelength of $13.4 \mathrm{~nm}$, and an acceptance half-angle of $48 \mu \mathrm{rad}$. 
interferogram $I(x, y)$ can be represented as the convolution of the Fourier transform of the Airy envelope with three delta functions. The delta functions arising from the $1+\cos (\cdot)$ term can be written as

$$
\delta\left(f_{x}, f_{y}\right)+\frac{\left|\boldsymbol{\mu}_{12}\right|}{2}\left[\delta\left(f_{x}+\frac{s}{\lambda z}, f_{y}\right)+\delta\left(f_{x}-\frac{s}{\lambda z}, f_{y}\right)\right] .
$$

Furthermore, the Fourier transform of the Airy envelope becomes the autocorrelation of the pinhole. The resultant pattern in the frequency domain is therefore one zeroth order peak and two symmetric first-order peaks, each properly scaled. Ideally, the fringe visibility is two times the relative strength of the first-order peak to the zerothorder peak. In practice, we apply properly displaced top-hat filters centered at each peak and integrate within the filters. The fringe visibility is then determined by two times the ratio of the integration under the first-order peak to that under the zeroth-order peak.

In Fig. 2.5 we show $\left|\boldsymbol{\mu}_{12}\right|$ as a function of pinhole separation for both horizontally and vertically separated pinholes. One observes that the transverse coherence distance in the vertical plane is greater than that in the horizontal plane, for this 48 - $\mu \mathrm{rad}$ acceptance NA. Following the convention in [3], a transverse coherence distance $L_{c}$ for the measured coherence profiles (Fig. 2.5) is obtained by determining the width of an equivalent top-hat function, i.e.,

$$
L_{c} \equiv \int_{-\infty}^{\infty}\left|\boldsymbol{\mu}_{12}(\Delta x)\right|^{2} d \Delta x .
$$

The measured transverse coherence distance in the horizontal direction, $L_{c, h}$, is found to be approximately $6.3 \mu \mathrm{m}$ and the measured transverse coherence distance in the vertical direction, $L_{c, v}$, is found to be approximately $7.4 \mu \mathrm{m}$. This is due to the fact that the vertical source dimension is sub-resolution in size at this acceptance angle, while the horizontal size is not. After propagating from the undulator exit (source), the FWHM of the spatial coherence profile, as calculated by the van Cittert-Zernike theorem, is $0.3 \mathrm{~mm}(H) \times 5.2 \mathrm{~mm}(V)$ at the beamline acceptance aperture. Therefore, the spatial coherence profile at the KB focal plane (image plane) is expected to be asymmetric with vertical coherence better than horizontal coherence. As will 

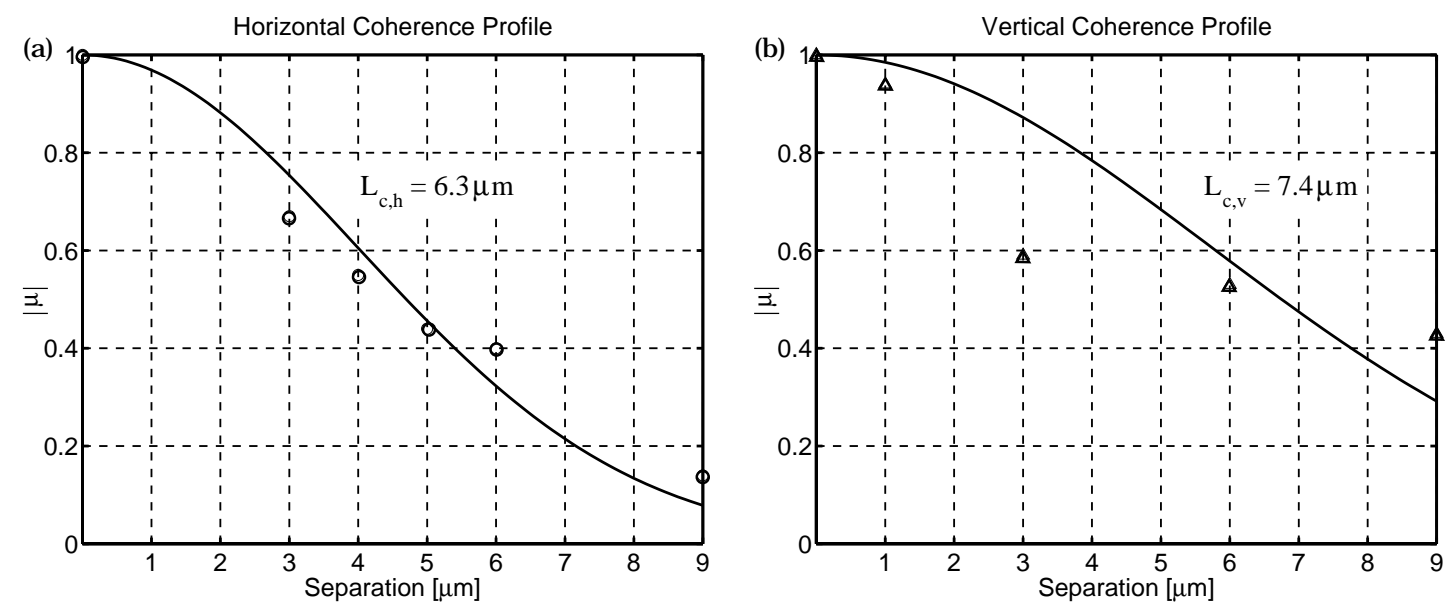

Figure 2.5: The measured coherence factor $\left|\boldsymbol{\mu}_{12}\right|$ as function of pinhole separation distance for (a), horizontally and (b), vertically separated pinholes. For these mea-

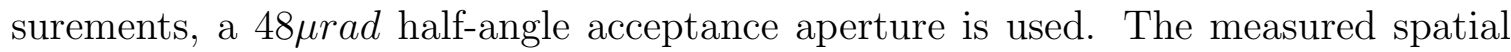
coherence distance decreases with larger pinhole separations as expected. Larger uncertainty in the vertical $3 \mu \mathrm{m}$ separation may be due to a smaller number of interferograms collected in this case. The data points are fitted to a Gaussian curve in each case.

be shown in Chapter 3, simple analysis of a critical illumination system like the one considered here would suggest the coherence to be symmetric when the NA is symmetric. More detailed analysis reveals, however, that the small vertical source size causes the vertical coherence width at the entrance pupil to be large relative to the pupil size. In this case we violate the assumptions typically used for predicting coherence in a critical illumination. This causes a preferential increase of coherence in the vertical direction.

The effect of radiation directly transmitted through the mask membrane, a source of noise in these measurements, can be seen in Fig. 2.3(d). This effect becomes more significant as the pinhole separation increases because the limited beam size (see Fig. 2.6) results in a reduced illumination intensity at each pinhole, whereas the directly transmitted radiation remains fixed. This directly transmitted light adds a background noise to the interference pattern, thus reducing fringe visibility locally in the affected region. Therefore, when applying the Fourier transform method to the cases of large pinhole separations, we avoid the region containing directly transmitted 
light. This Fourier transform method has the advantage of evaluating the fringe visibility as an integrated, rather than localized, property of the full interferogram.

The intensity and coherence distribution in the KB focal plane is calculated by way of computer simulation for an aberration-free beamline. The simulation shows that the coherence profile is wider than the calculated intensity profile for the aberrationfree beam in the vertical direction, which means that without aberrations the beam would be essentially fully coherent in the vertical direction. Horizontally, the coherence profile is dominated by the acceptance NA. As described above, this asymmetry is expected based on the geometry of the system.

Figure 2.6 displays both FWHM intensity contours and $\left|\boldsymbol{\mu}_{12}\right|=0.5$ isometrics for both the aberration-free simulation case, and the actual measured results. In both cases the vertical coherence is seen to be larger than the beam vertical FWHM, indicating nearly complete coherence in the vertical direction. Also both simulation and experiment show the horizontal coherence to be smaller than the vertical coherence and significantly smaller than the beam. This is a result of the extended source in the horizontal direction and the beamline geometry. The results differ, however, in that the measured coherence and beam-size are larger than those predicted for the aberration-free simulation. The beam-size increase is attributed to aberrations in the KB. By comparing the two intensity profiles we surmise the aberration limited point-spread function of the KB to be about $4 \mu \mathrm{m}$ in diameter. We assume these aberrations to also play a role in the increased coherence observed experimentally. 


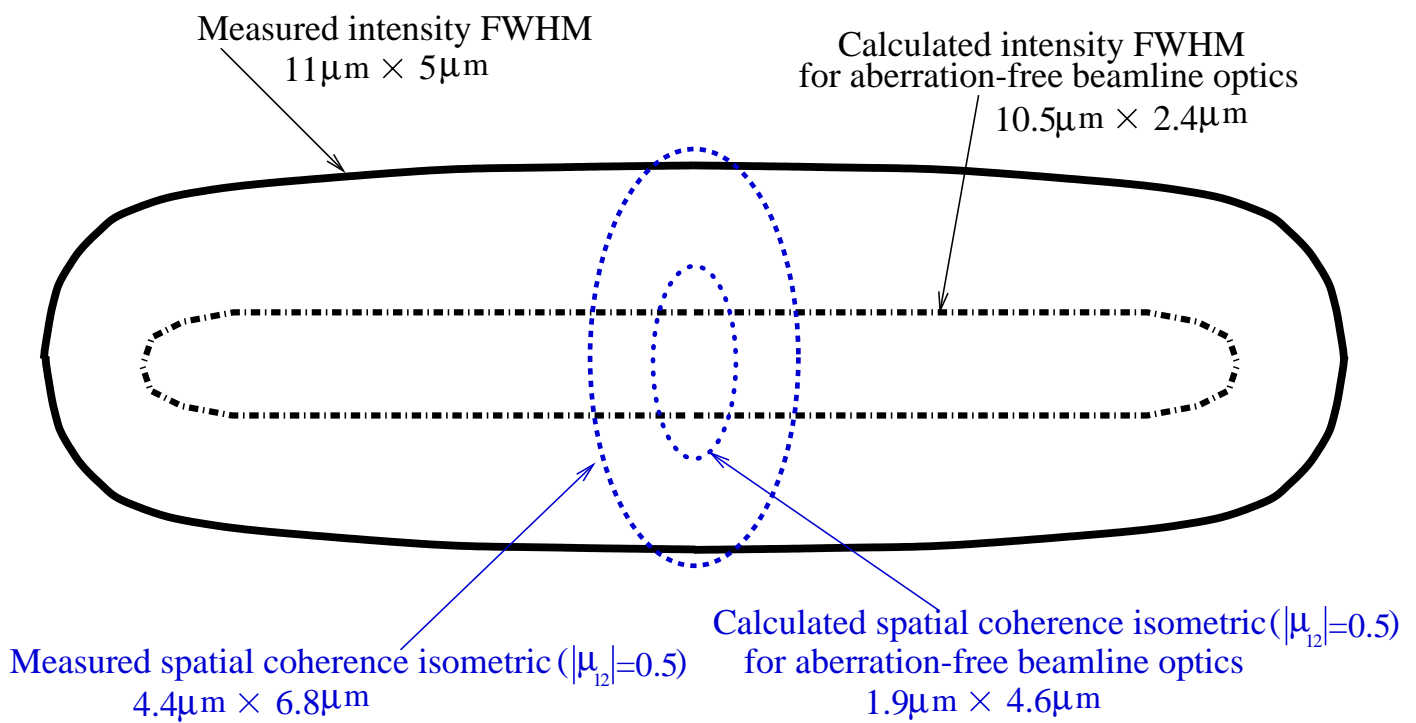

Figure 2.6: The beamline is designed to image the undulator output to the KB focus with a demagnification of 60 and an acceptance half-angle of $48 \mu \mathrm{rad}$. The undulator output in this calculation is assumed to be a monochromatic $(\lambda=13.4 \mathrm{~nm})$, spatially incoherent Gaussian-shaped source with $\left(\sigma_{x}, \sigma_{y}\right)=(260 \mu m, 16 \mu m)$ [8], corresponding to source plane values of $612 \mu \mathrm{m} \times 38 \mu \mathrm{m}$ FWHM. The solid line shows the FWHM of the measured KB focal intensity distribution. The dash-dot line is the FWHM of this calculated $\mathrm{KB}$ focal intensity distribution assuming an aberration-free beamline. The intensity distribution FWHM values are increased by aberrations from ideal values of $10.5 \mu m(H) \times 2.4 \mu m(V)$, to experimental values of $11 \mu m(H) \times 5 \mu m(V)$. The dashed line represents the calculated focal plane spatial coherence isometric $\left(\left|\boldsymbol{\mu}_{12}\right|=0.5\right)$ for experimental values of wavelength, acceptance NA, and demagnification, as calculated using the van Cittert-Zernike theorem $[1,3]$. The asymmetric coherence isometric is due to the asymmetry of the source intensity distribution. 


\subsubsection{Effect of monochromator exit-slit}

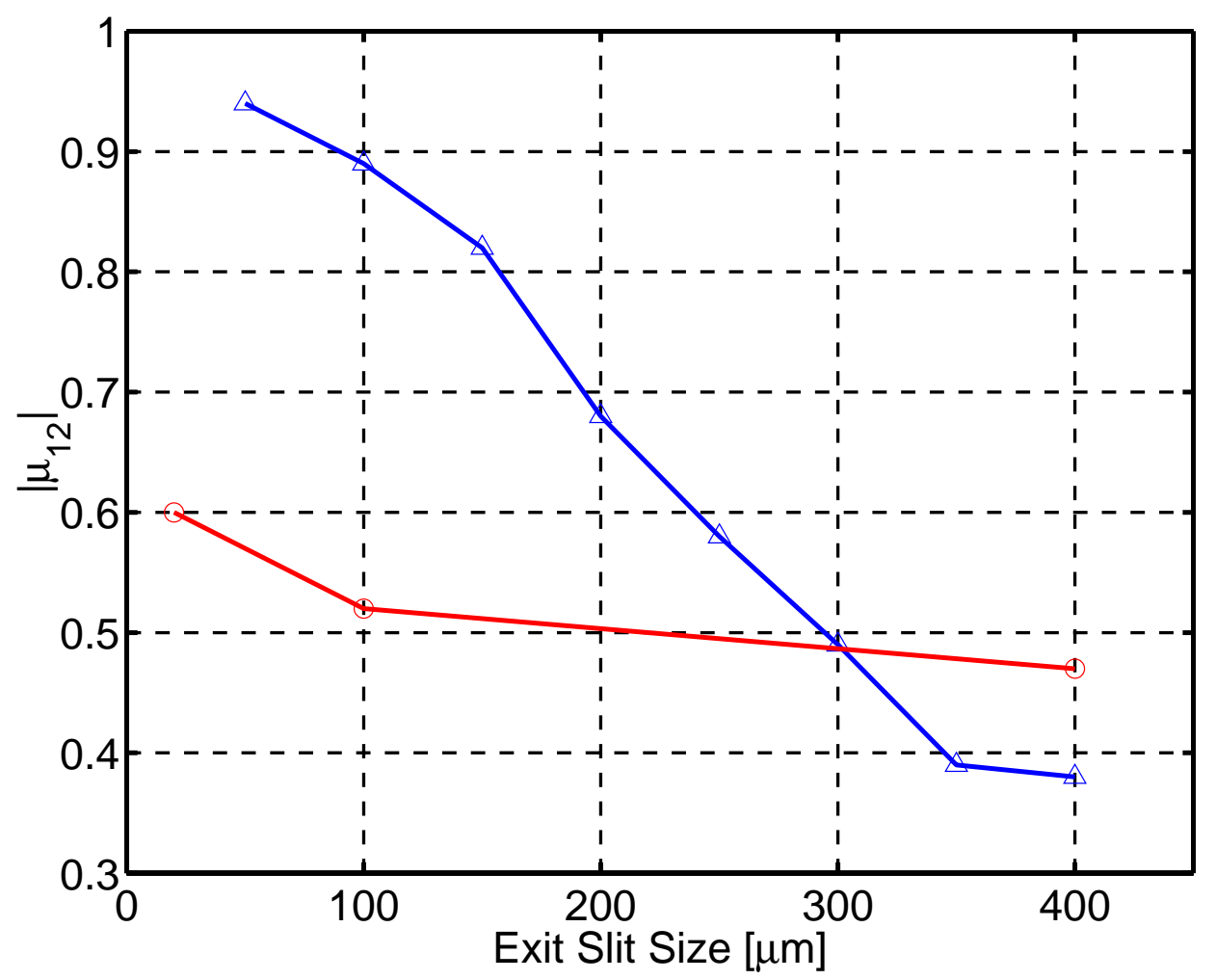

Figure 2.7: The vertical opening of monochromator's exit-slit has significant effect on the vertical coherence but not on the horizontal coherence. A smaller exit-slit size decreases the vertical phase-space of the transmitted radiation, thus increasing the vertical coherence. Horizontal coherence is relatively unaffected. This is done with a larger beamline acceptance NA.

The natural bandwidth of undulator radiation within the central radiation cone, $\theta_{c e n}$, is set by the number of magnet pairs $N$ [8], which is 55 in these experiments. The monochromator is designed to transmit a bandpass variable from $\lambda / \Delta \lambda=55$ to 1100. The larger value is useful in experiments requiring a longer coherence length (to $7.4 \mu \mathrm{m}$ at $13.4 \mathrm{~nm}$ wavelength). These values of spectral bandpass correspond to exit-slit widths of $320 \mu \mathrm{m}$ and $16 \mu \mathrm{m}$, respectively. Exit-slit size greater than $320 \mu \mathrm{m}$ does not further affect the monochromator bandpass. All data presented to this point correspond to a $400 \mu \mathrm{m}$ exit-slit size $(\lambda / \Delta \lambda=55)$. Use of a smaller monochromator 
exit-slit narrows the transmitted spectrum, but also has the effect of decreasing the transmitted beam's transverse phase-space, and thus increasing the spatial coherence length in the vertical plane. To study the effect of the monochromator exit-slit size, we have repeated the experiment with exit-slit size as a parameter. Figure 2.7 shows the measured horizontal and vertical coherence as a function of monochromator exitslit size. For a vertical pinhole separation of $6 \mu \mathrm{m}$, the fringe visibility varies from 0.38 to 0.94 as the exit-slit size changes from $400 \mu \mathrm{m}$ to $50 \mu \mathrm{m}$. For the horizontally oriented $4-\mu m$ separation pinholes, the fringe visibility varies from 0.47 to 0.60 as the exit-slit size changes from $400 \mu \mathrm{m}$ to $20 \mu \mathrm{m}$. As one expects, the exit-slit also acts as a spatial filter, having a significant effect on spatial coherence in the vertical plane, and minimal effect in the horizontal plane.

\subsubsection{Wave-front null test}

The two-pinhole experiment presented here can also be used to measure the departure from sphericity of the pinhole-diffracted wave. Figure 2.8 is derived from the measured interference pattern obtained with 450 - $\mu m$ diameter pinholes horizontally placed $9 \mu \mathrm{m}$ apart. To determine the underlying wave-front quality of the two nearly spherical waves used to produce the interference pattern, the interferogram is analyzed using conventional Fourier-transform wave-front reconstruction techniques routinely applied to carrier-frequency interferograms [44]. The resulting wave-front is then compared to what one would expect from two perfectly spherical waves in our recording geometry. The rms departure from a sphere is taken to represent the underlying pinhole-diffracted wave-front quality. For example, at a numerical aperture of 0.025 (a typical input numerical aperture for testing EUV optics), the wave-front quality from these $450-\mu m$ pinholes is seen to be $\lambda / 330$, exceeding current requirements for such tests $[41,45,46]$. 


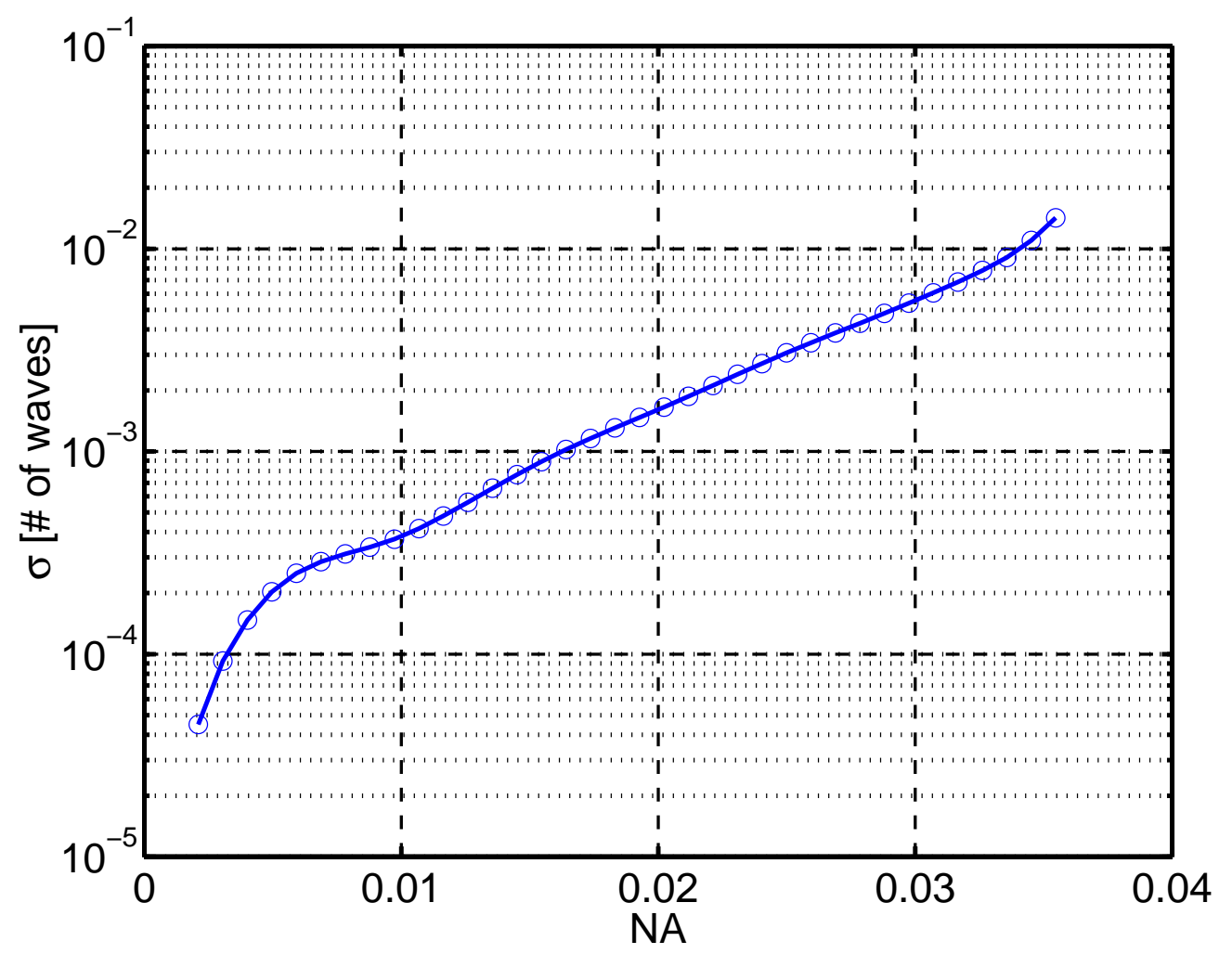

Figure 2.8: Departure of pinhole generated wave-front from sphericity, expressed as an rms number of waves at $\lambda=13.4 \mathrm{~nm}$. For example, the data indicates that with 450- $\mathrm{nm}$-pinhole filtered radiation a wave-front departure from a sphericity of $\lambda / 1000$ is obtained across a wave-front of about $0.016 \mathrm{NA}$ (equal to 13 picometer rms at this wavelength and NA).

\subsection{Conclusion}

The coherence properties of spatially filtered undulator radiation have been measured. A very high degree of spatial coherence is demonstrated, as expected on the basis of a simple model. The effect of an asymmetric source size on the resultant coherence properties is observed, and is consistent with aperturing within the beamline optical system used to transport radiation to the experimental chamber. Based on these observations and well understood scaling of undulator radiation, it is evident that high average power, spatially coherent radiation is available at modern storage rings with the use of appropriate pinhole spatial filtering techniques. The ability 
of synchrotron facilities to provide high spatial coherence at hard x-ray region has recently been confirmed as expected $[32,47]$. 


\title{
Chapter 3
}

\section{Analysis of the illumination}

\section{coherence properties of systems}

\author{
where the Van Cittert-Zernike
}

\section{theorem does not apply}

\begin{abstract}
Modern synchrotron beamlines often take the form of critical illumination systems, where an incoherent source of limited spatial extent is re-imaged to some experimental plane of interest. Unique constraints of these sources and beamlines, however, may preclude the use of the simple Van Cittert-Zernike theorem for calculating the object-image coherence relationship. Here, a rigorous analysis of the object-image coherence relationship valid for synchrotron beamlines is performed. The analysis shows beamline aberrations to have an effect on the coherence properties. Effects of various low-order aberrations on the coherence are explicitly studied.
\end{abstract}

\subsection{Introduction}

High brightness and high coherent power undulators available at third generation synchrotron radiation facilities, through spatial and spectral filtering, enable a variety of experiments that require a high degree of coherence at short wavelengths. 
Undulator radiation, at its exit-plane, is regarded as an incoherent source because the electrons in the storage ring have uncorrelated motion and thus are essentially independent radiators. Beamline optics are routinely used to re-image this spatially confined incoherent source to an experimental plane of interest. Such a configuration is readily recognized as a critical illumination system [1], where the beamline acts as the condenser.

Undulator radiation has an intrinsic divergence angle, known as the central radiation cone angle $\theta_{c e n}$, characterized by the electron's forward-emitting radiation. The beamline acceptance angle, i.e. object-side NA of the condenser, is usually set comparable but slightly smaller than $\theta_{c e n}$. This acceptance angle sets the effective coherence patch size on the source as seen by the condenser system. For the Van Cittert-Zernike theorem to correctly predict the spatial coherence distribution at the image plane of the condenser, the dimension of the source needs to be much greater than this effective coherence patch size, i.e.

$$
d_{\text {source }} \gg d_{\text {coh }}^{\text {eff }} \approx \frac{1}{2 \pi} \frac{\lambda}{\theta_{\text {accept }}}
$$

where $d_{\text {source }}$ is the source dimension, $d_{c o h}^{e f f}$ is the effective coherence patch size at the source, $\lambda$ is the wavelength, and $\theta_{\text {accept }}$ is the beamline acceptance angle. However, the distinct characteristics of third generation undulator radiation, i.e. the small vertical source dimension and the constraint on the size of beamline acceptance angle, give rise to a condenser system whose spatial coherence properties cannot be simplified by the commonly used Zernike approximation [1,3,6]. For the $\lambda=13.4 \mathrm{~nm}$ experiments described here, the Gaussian undulator source has a vertical dimension $(2 \sigma)$ of $d_{\text {source }}=32 \mu \mathrm{m}$, and the beamline acceptance angle is $\theta_{\text {accept }}=48 \mu \mathrm{rad}$, slightly smaller than the central radiation cone angle of $80 \mu \mathrm{rad}$. The $d_{\text {coh }}^{\text {eff }}$ thus equals to $45 \mu \mathrm{m}$, for which Eq.(3.1) is clearly not satisfied and the Zernike approximation is not applicable.

Here, based on the Huygens-Fresnel principle, the analysis and numerical evaluation of the spatial coherence properties of a representative undulator beamline are presented and the results are compared with actual measurements conducted at the ALS undulator beamline 12 . 


\subsection{Rigorous mutual coherence propagation for un- dulator beamlines}

\subsubsection{Undulator radiation as an incoherent source}

The undulator radiation at its exit-plane is assumed to be an incoherent source here. In practice, this assumption is valid as long as the coherence width at the source is smaller than the diffraction-limited resolution of the condenser. Alternatively, this could be stated as requiring the intrinsic divergence of the source to be larger than the acceptance angle of the condenser. For the undulator source, the positions and motion of the electrons in the undulator are uncorrelated. All point radiators originating from uncorrelated electrons can be treated as independent, and the size of an elemental point radiator can be determined from the central radiation cone divergence [8]. The intrinsic divergence of the EUV undulator discussed here is $\theta_{\text {cen }}=80 \mu \mathrm{rad}$, which is larger than the beamline acceptance angle $\theta_{\text {accept }}$ of $48 \mu \mathrm{rad}$. Therefore, it is evident that the incoherent source approximation holds here.

\subsubsection{Zernike approximation for a condenser system}

The Zernike approximation, first described by F. Zernike in 1938 [6], states that the condenser lens pupil, when illuminated by a large incoherent source, can be regarded as a secondary incoherent source whose intensity distribution is given by the modulus square of the pupil function. This approximation, discussed again by Born \& Wolf [1] (sec.10.5.2) and Goodman [3] (sec.7.2.2), is commonly used for condensers operating at visible wavelengths.

Starting with an incoherent source placed at the object plane of the condenser, the Van Cittert-Zernike theorem $[1,3,6]$ can be used to propagate this incoherent source to the condenser lens pupil and the resultant mutual intensity at the condenser lens is given by a Fourier transform of the source intensity distribution. The condition under which the Zernike approximation is valid requires that the incoherent source subtends a sufficiently large angle at the condenser lens, such that the coherence 
width at the condenser lens is small relative to the pupil diameter. Satisfying this condition, the condenser lens pupil can be regarded as a secondary source with a very small coherence area and the generalized Van Cittert-Zernike theorem [3] can then be used to propagate the mutual intensity from the condenser lens pupil to the image plane of the condenser. The resultant coherence distribution at the image plane of the condenser is thus determined solely by the modulus square of the pupil function and aberrations in the condenser lens do not affect the coherence distribution at the image plane [3]. Note that in this case, the resultant intensity distribution at the image plane of the condenser is determined by the coherence function at the lens pupil.

However, when the dimension of the incoherent source shrinks to the point where the coherence width at the condenser lens is comparable to the pupil diameter, the Zernike approximation fails and the generalized Van Cittert-Zernike theorem can no longer be used to propagate the mutual intensity function from the exit of the condenser lens to the image-plane. Under this small-source condition, a rigorous mutual coherence propagation based on the Huygens-Fresnel principle is required [3]. As will be shown later in this chapter, the condenser pupil aberrations in this case begin to affect the coherence properties at the condenser image-plane.

\subsubsection{Undulator beamline as a condenser: an example}

Undulator Beamline 12 of the ALS can be viewed as an incoherent source with a Gaussian intensity distribution, $\left(\sigma_{\xi}, \sigma_{\eta}\right)=(260 \mu m, 16 \mu m)$. The beamline essentially acts as a condenser lens with a de-magnification of 60 and an object-side NA of $48 \mu \mathrm{rad}$. The distance $z_{1}$ from the exit-plane of the undulator to the pupil is $16.7 \mathrm{~m}$ and the pupil radius $a$ is $0.8 \mathrm{~mm}$. The wavelength used here is $\lambda=13.4 \mathrm{~nm}$.

Using the Van Cittert-Zernike theorem to propagate radiation from the incoherent source to the lens pupil, the mutual intensity at the pupil is given by a Fourier transform of the Gaussian intensity distribution of the incoherent undulator source (see Appendix A). The resultant coherence distribution at the pupil is then Gaussian 
distributed with rms radii

$$
\left(\sigma_{x}^{C}, \sigma_{y}^{C}\right)=\left(\frac{\lambda z_{1}}{2 \pi \sigma_{\xi}}, \frac{\lambda z_{1}}{2 \pi \sigma_{\eta}}\right) \approx(0.14 \mathrm{~mm}, 2.23 \mathrm{~mm}) .
$$

Comparing the vertical size of this coherence patch with the pupil diameter $(2 a=$ $1.6 \mathrm{~mm}$ ), the Zernike approximation is found not to be applicable here and therefore the generalized Van Cittert-Zernike theorem cannot be used to propagate the mutual coherence from the condenser pupil to the image-plane. However, should this invalidity be ignored and the Zernike approximation used for the calculation of the spatial coherence distribution at the condenser image-plane, the erroneous resultant coherence distribution would be an Airy pattern with a first null radius $s_{o}$ of $2.84 \mu \mathrm{m}$. Specifically, the modulus of the complex coherence factor would be

$$
\left|\mu_{12}\right|=2 \frac{J_{1}\left(\frac{2 \pi a}{\lambda z_{2}} \sqrt{(\Delta u)^{2}+(\Delta v)^{2}}\right)}{\frac{2 \pi a}{\lambda z_{2}} \sqrt{(\Delta u)^{2}+(\Delta v)^{2}}}
$$

where $a$ is the pupil radius, $\lambda$ is the wavelength, $z_{2}$ is the distance from the condenser lens to the image-plane, $(\Delta u, \Delta v)$ is the coordinate difference at the image plane, and the first null radius is $s_{o}=0.610 \lambda z_{2} / a$. As expected, this over-simplification results in discrepancy with the experimentally measured coherence profile [9], which determined the size of the coherence patch to be $4.4 \mu \mathrm{m}$ and $6.8 \mu \mathrm{m}$ in the horizontal and vertical direction, respectively.

As demonstrated, the generalized Van Cittert-Zernike theorem does not apply here. A rigorous analysis on mutual coherence propagation using the Huygens-Fresnel principle and subsequent numerical evaluations are presented in this chapter. 


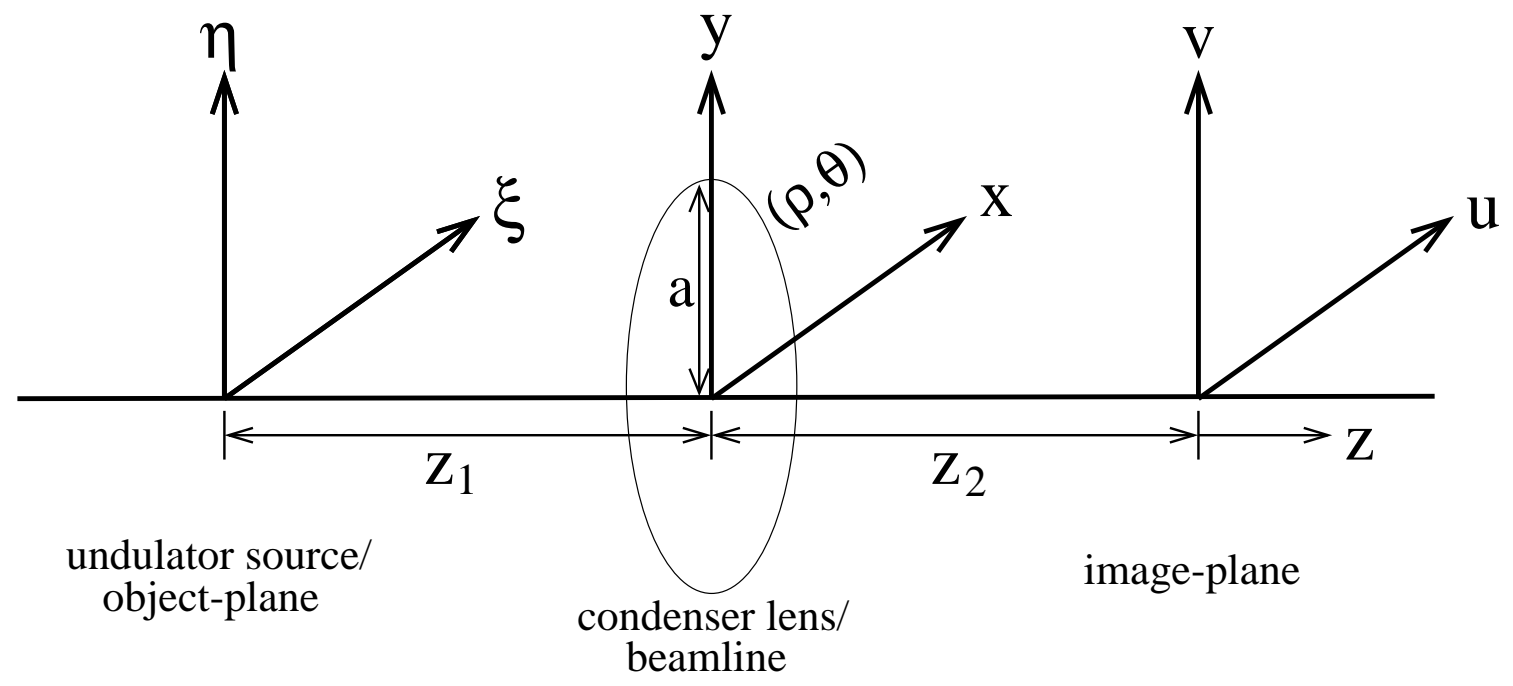

Figure 3.1: Coordinate system

\subsection{Object-image coherence relation}

Using the Huygens-Fresnel principle [3], the object-image coherence relation, under quasi-monochromatic and paraxial approximations, can be expressed as

$\mathbf{J}_{i}\left(u_{1}, v_{1} ; u_{2}, v_{2}\right)=\iiint_{-\infty}^{+\infty} \int_{\mathbf{J}_{o}}\left(\xi_{1}, \eta_{1} ; \xi_{2}, \eta_{2}\right) \mathbf{K}\left(u_{1}, v_{1} ; \xi_{1}, \eta_{1}\right) \mathbf{K}^{*}\left(u_{2}, v_{2} ; \xi_{2}, \eta_{2}\right) d \xi_{1} d \eta_{1} d \xi_{2} d \eta_{2}$

where $\mathbf{J}_{i}$ and $\mathbf{J}_{o}$ are the mutual intensities at the image and object plane, respectively. The $a \quad r a \quad \mathbf{K}$ is defined by

$$
\begin{aligned}
\mathbf{K}(u, v ; \xi, \eta)= & \frac{\exp \left\{j \frac{\pi}{\lambda z_{2}}\left(u^{2}+v^{2}\right)\right\} \exp \left\{j \frac{\pi}{\lambda z_{1}}\left(\xi^{2}+\eta^{2}\right)\right\}}{\lambda^{2} z_{2} z_{1}} \\
& \times \int_{-\infty}^{+\infty} \mathbf{P}(x, y) \exp \left\{-j \frac{2 \pi}{\lambda z_{2}}\left[\left(u+\frac{z_{2}}{z_{1}} \xi\right) x+\left(v+\frac{z_{2}}{z_{1}} \eta\right) y\right]\right\} d x d y
\end{aligned}
$$

where $\mathbf{P}(x, y)$ is the complex pupil function described in detail in Appendix B. The coordinate system used throughout this chapter is depicted in Fig. 3.1. Notice that the subscript $i$ for $\left(u_{i}, v_{i}\right)$ and $\left(\xi_{i}, \eta_{i}\right)$ is dropped in Eq.(3.3) for ease of notation. 
Also notice that the integral above can be regarded as a Fourier transform of the condenser lens pupil evaluated at spatial frequency $\left[\frac{1}{\lambda z_{2}}\left(u_{i}+M \xi_{i}\right), \frac{1}{\lambda z_{2}}\left(v_{i}+M \eta_{i}\right)\right]$, where $M=z_{2} / z_{1}$ and $i=1,2$.

If an incoherent source is placed at the object plane, then $\mathbf{J}_{o}$ can be written as

$$
\mathbf{J}_{o}=\kappa I_{s}(\xi, \eta) \delta(\Delta \xi, \Delta \eta)
$$

where $I_{s}(\xi, \eta)$ is the source intensity distribution, $\kappa=\lambda^{2} / \pi$, and $\delta(\cdot, \cdot)$ is a 2 dimensional Dirac delta function.

In this case, Eq.(3.2) simplifies to

$$
\mathbf{J}_{i}\left(u_{1}, v_{1} ; u_{2}, v_{2}\right)=\kappa \int_{-\infty}^{+\infty} \int_{s}(\xi, \eta) \mathbf{K}\left(u_{1}, v_{1} ; \xi, \eta\right) \mathbf{K}^{*}\left(u_{2}, v_{2} ; \xi, \eta\right) d \xi d \eta
$$

The mutual intensity function $\mathbf{J}_{i}$ at the image plane can now be determined by the integration of the source intensity distribution $I_{s}$ and the two off-centered [by $\left(u_{1}, v_{1}\right)$ and $\left(u_{2}, v_{2}\right)$, respectively] amplitude spread functions of the pupil $\mathbf{P}$.

In order to simplify the notation in Eq.(3.5), we define

$$
\mathbf{G}\left(u^{\prime}, v^{\prime}\right) \equiv \int_{-\infty}^{+\infty} \mathbf{P}(x, y) \exp \left\{-j \frac{2 \pi}{\lambda z_{2}}\left[u^{\prime} x+v^{\prime} y\right]\right\} d x d y
$$

Note that $\mathbf{G}\left(u^{\prime}, v^{\prime}\right)$ is essentially the point-spread function (up to a scaling constant) of the pupil $\mathbf{P}(x, y)$. Eq.(3.5) can now be written as

$$
\begin{aligned}
\mathbf{J}_{i}\left(u_{1}, v_{1} ; u_{2}, v_{2}\right)= & \frac{\kappa \exp \left\{j \frac{\pi}{\lambda z_{2}}\left(u_{1}^{2}+v_{1}^{2}-u_{2}^{2}-v_{2}^{2}\right)\right\}}{\lambda^{4} z_{2}^{2} z_{1}^{2}} \\
& \times \iint_{-\infty}^{+\infty} I_{s}(\xi, \eta) \mathbf{G}\left(u_{1}+M \xi, v_{1}+M \eta\right) \mathbf{G}^{*}\left(u_{2}+M \xi, v_{2}+M \eta\right) d \xi d \eta,
\end{aligned}
$$

The mutual intensity can be obtained by numerically evaluating the above double integral. Equation (3.7), and its equivalent Eq.(3.5), are based on the Huygens-Fresnel principle and are valid regardless of the Zernike approximation. Note here that the 
mutual intensity is a function of the four individual coordinates, $\left(u_{1}, v_{1}, u_{2}, v_{2}\right)$, not their differences.

The numerical value of $\mathbf{G}\left(u^{\prime}, v^{\prime}\right)$ can be determined by evaluating the integral in Eq.(3.6). As will be shown in Section 3.4, this integral can be expressed as weighed summations of various Bessel functions for the specific aberrations involved. Defocus/distortion, astigmatism, and coma are the low order aberrations whose effects on the spatial coherence distributions at the condenser image plane are studied here.

\subsubsection{Zernike approximation}

Before using Eq.(3.7), or equivalently Eq.(3.5), it is interesting to examine the Zernike approximation and the conditions under which it is valid. The Zernike approximation [6] states that the coherence distribution at the image plane of a condenser that re-images the source can be determined solely by the modulus square of the pupil function, thus independent of the pupil aberrations. Examining Eq.(3.5) [the equivalent of Eq.(3.7)], which is valid in general for all incoherent sources, one finds that Eq.(3.5) reduces to the commonly known Zernike approximation when the size of the incoherent source is large enough such that $I_{s}(\xi, \eta)$ can be effectively regarded as a constant $\mathbb{C}$. To demonstrate this, substitute the amplitude spread function $\mathbf{K}(u, v ; \xi, \eta)$ in Eq. (3.5) with Eq.(3.3), and integrate first $d \xi d \eta$ with $I_{s}(\xi, \eta)=\mathbb{C}$. This yields

$$
\begin{aligned}
& \mathbf{J}_{i}\left(u_{1}, v_{1} ; u_{2}, v_{2}\right)= \mathbb{C} \frac{\kappa \exp \left\{j \frac{\pi}{\lambda z_{2}}\left(u_{1}^{2}+v_{1}^{2}-u_{2}^{2}-v_{2}^{2}\right)\right\}}{\lambda^{4} z_{2}^{2} z_{1}^{2}} \\
& \times \iint_{-\infty}^{+\infty} \mathbf{P}\left(x_{1}, y_{1}\right) d x_{1} d y_{1} \iint_{-\infty}^{+\infty} \mathbf{P}^{*}\left(x_{2}, y_{2}\right) d x_{2} d y_{2} \\
& \quad \times \int_{-\infty}^{+\infty} d \xi d \eta \exp \left\{-j \frac{2 \pi}{\lambda z_{2}}\left[\left(u_{1}+\frac{z_{2}}{z_{1}} \xi\right) x_{1}+\left(v_{1}+\frac{z_{2}}{z_{1}} \eta\right) y_{1}\right]\right\} \exp \left\{j \frac{2 \pi}{\lambda z_{2}}\left[\left(u_{2}+\frac{z_{2}}{z_{1}} \xi\right) x_{2}+\left(v_{2}+\frac{z_{2}}{z_{1}} \eta\right) y_{2}\right]\right\} .
\end{aligned}
$$

Note that the last integral in the above equation evaluates to

$$
\exp \left\{-j \frac{2 \pi}{\lambda z_{2}}\left[u_{1} x_{1}+v_{1} y_{1}-u_{2} x_{2}-v_{2} y_{2}\right]\right\} \times \delta\left(\frac{x_{1}-x_{2}}{\lambda z_{1}}, \frac{x_{1}-x_{2}}{\lambda z_{1}}\right)
$$


Further integrate over $d x_{2} d y_{2}$ to yield

$$
\begin{aligned}
\mathbf{J}_{i}\left(u_{1}, v_{1} ; u_{2}, v_{2}\right)= & \frac{\kappa^{\prime} \exp \left\{j \frac{\pi}{\lambda z_{2}}\left(u_{1}^{2}+v_{1}^{2}-u_{2}^{2}-v_{2}^{2}\right)\right\}}{\lambda^{2} z_{2}^{2}} \\
& \times \int_{-\infty}^{+\infty} d x_{1} d y_{1}\left|\mathbf{P}\left(x_{1}, y_{1}\right)\right|^{2} \exp \left\{-j \frac{2 \pi}{\lambda z_{2}}\left[\left(u_{1}-u_{2}\right) x_{1}+\left(v_{1}-v_{2}\right) y_{1}\right]\right\}
\end{aligned}
$$

where $\kappa^{\prime}=\kappa \mathbb{C}$.

Eq.(3.9) indeed shows that in the case where $I_{s}$ is large enough, the image-plane mutual intensity $\mathbf{J}_{i}$ depends only on the modulus square of the pupil function (thus independent of the aberrations in the pupil) and its magnitude, $\left|\mathbf{J}_{i}\right|$, is a function of $(\Delta u, \Delta v)$ only. Also shown in Eq.(3.9), the image-plane mutual intensity is essentially a Fourier transform of the modulus square of the pupil. Note that Eq.(3.9) is identical to (7.2-17) in Ref. [3], which was obtained by explicitly assuming a Dirac- $\delta$-function coherence distribution at the condenser lens plane.

Validity of the Zernike approximation In arriving at Eq.(3.9), the assumption was made that the incoherent source was of infinite extent with uniform intensity distribution $\mathbb{C}$. However, as mentioned in Section 3.2, this assumption can be relaxed to that the size of the incoherent source be large enough such that the coherence patch at the entrance of the condenser lens is sufficiently smaller than the lens pupil. Under this relaxed condition, the generalized Van Cittert-Zernike theorem is applicable at exit of the the condenser lens and Eq.(3.9) still holds with some modification on $\kappa^{\prime}$. In this case, the $\kappa^{\prime}$ in Eq.(3.9) is actually a function of $(\bar{u}, \bar{v})=\left(\frac{u_{1}+u_{2}}{2}, \frac{v_{1}+v_{2}}{2}\right)$, given by

$$
\kappa^{\prime}(\bar{u}, \bar{v})=\kappa I_{s}\left(-\frac{z_{1}}{z_{2}} \bar{u},-\frac{z_{1}}{z_{2}} \bar{v}\right)
$$

Note that when the incoherent source is of infinite extent with uniform intensity distribution $\mathbb{C}$, the resultant $\kappa^{\prime}$ indeed reduces to $\kappa^{\prime}=\kappa \mathbb{C}$. In practice, the incoherent source cannot be infinitely large, and using the simpler form of Eq.(3.9) where $\kappa^{\prime}$ is a constant requires caution. In fact, the geometric image of the source needs to be sufficiently larger than the object of interest, in order for the intensity of the 
illumination on the object to be regarded as uniform and Eq.(3.9) to strictly apply. Mathematically, this condition can seen from Eq.(3.10) as

$$
\left\{\begin{array}{l}
\left|\frac{z_{1}}{z_{2}} u_{o b j}\right| \ll\left|\xi_{s r c}\right| \\
\left|\frac{z_{1}}{z_{2}} v_{o b j}\right| \ll\left|\eta_{s r c}\right|
\end{array}\right.
$$

where $\left(u_{o b j}, v_{o b j}\right)$ and $\left(\xi_{s r c}, \eta_{s r c}\right)$ denote the maximum dimension of the object under illumination and that of the incoherent source, respectively. Therefore, when applying Eq.(3.9) to describe the mutual intensity incident on an object, the additional requirement on the relative size of the object under illumination and the geometric image of the source must be satisfied.

Although valid in a wide array of situations, the simple Fourier transform relationship, Eq.(3.9), established by the Zernike approximation, breaks down as the dimension of the incoherent source shrinks and the coherence area at the pupil plane increases. As described above, this small-source condition is the norm for the vertical dimension with undulator radiation. Therefore, one cannot resort to the Zernike approximation here and numerical evaluation based on Eq.(3.7) in Sec.3.3 is needed for examining the mutual intensity distribution at the condenser image-plane.

\subsection{Point spread functions for aberrated pupils}

To proceed on the numerical evaluation using Eq.(3.7), it is evident that the expressions for the PSF (i.e. G) corresponding to various low-order aberrations are needed. In this section, PSFs for defocus/distortion, astigmatism, and coma, are presented.

\subsubsection{Distortion \& Defocus}

The displacement theorem, as presented in Ref. [1] in terms of , states that for any two arbitrary aberration functions whose difference is given by $H \rho^{2}+$ $K \rho \sin \theta+L \rho \cos \theta+N$, where $H, K, L$, and $N$ are constants of order $\lambda$, their respective distributions near focus are identical apart from a displacement 
described by the transformation given later in Eq.(3.20). Resembling the derivation of the displacement theorem in Ref. [1], the same theorem in terms of , i.e. PSF, for the two arbitrary aberration functions differing by $H \rho^{2}+K \rho \sin \theta+L \rho \cos \theta+N$, will also be established in Eq.(3.23). Notice that the allowed difference between the two aberration functions, $H \rho^{2}+K \rho \sin \theta+L \rho \cos \theta+N$, is essentially distortion and defocus. Therefore, by setting one of the pupil functions to zero, the PSFs of distortion and defocus can be obtained from an un-aberrated PSF, which in turn can be asymptotically approximated by a series of Bessel functions.

\section{The displacement theorem}

When distortion and defocus are the only difference between two pupil functions, their respective PSFs are related simply by translation perpendicular and parallel to the optical axis. This is called the displacement theorem. The field distribution due to an arbitrary aberration function $\Phi$ can be expressed as

$$
U(P)=-\frac{i}{\lambda} \frac{a^{2} A}{z_{2}^{2}} e^{i\left(z_{2} / a\right)^{2} \zeta} \int_{0}^{1} \int_{0}^{2 \pi} \exp \left\{i\left[k \Phi-\nu \rho \cos (\theta-\phi)-\frac{1}{2} \zeta \rho^{2}\right]\right\} \rho d \rho d \theta
$$

where $a$ is the radius of the exit-pupil, $A$ is a scaling factor indicating the strength of the beam, $\rho$ is the normalized (with respect to $a$ ) radial coordinate at the exit-pupil plane, and

$$
\begin{aligned}
\zeta & =\frac{2 \pi}{\lambda}\left(\frac{a}{z_{2}}\right)^{2} z \\
\nu & =\frac{2 \pi}{\lambda}\left(\frac{a}{z_{2}}\right) \sqrt{u^{2}+v^{2}} \\
\phi & =\arctan \frac{v}{u} .
\end{aligned}
$$

For ease of notation we define

$$
f(\zeta, \nu, \phi ; \rho, \theta)=k \Phi-\nu \rho \cos (\theta-\phi)-\frac{1}{2} \zeta \rho^{2}
$$

Assuming a second aberration function $\Phi^{\prime}$ which differs from $\Phi$ only by distortion and defocus, i.e.

$$
\Phi^{\prime}=\Phi+H \rho^{2}+K \rho \sin \theta+L \rho \cos \theta+N
$$


where $H$ is the magnitude of the defocus, $K$ and $L$ are the $x$ and $y$ distortion magnitudes, respectively, then we have

$$
\begin{aligned}
& f(\zeta, \nu, \phi ; \rho, \theta)=k \Phi^{\prime}-\frac{1}{2}(2 k H+\zeta) \rho^{2}-\rho \cos \theta(\nu \cos \phi+k L)-\rho \sin \theta(\nu \sin \phi+k K)-k N \\
& =k \Phi^{\prime}-\frac{1}{2}(2 k H+\zeta) \rho^{2}-k N- \\
& \rho \sqrt{(\nu \cos \phi+k L)^{2}+(\nu \sin \phi+k K)^{2}}\left(\cos \theta \frac{\nu \cos \phi+k L}{\sqrt{(\cdot)^{2}+(\cdot)^{2}}}+\sin \theta \frac{\nu \sin \phi+k K}{\sqrt{(\cdot)^{2}+(\cdot)^{2}}}\right) \\
& =k \Phi^{\prime}-\nu^{\prime} \rho \cos \left(\theta-\phi^{\prime}\right)-\frac{1}{2} \zeta^{\prime} \rho^{2}-k N
\end{aligned}
$$

where

$$
\begin{aligned}
\zeta^{\prime} & =\zeta+2 k H & & \\
\nu^{\prime} & =\sqrt{(\nu \cos \phi+k L)^{2}+(\nu \sin \phi+k K)^{2}} & \nu^{\prime} \cos \phi^{\prime} & =\nu \cos \phi+k L \\
\phi^{\prime} & =\arctan \frac{\nu \sin \phi+k K}{\nu \cos \phi+k L} & \nu^{\prime} \sin \phi^{\prime} & =\nu \sin \phi+k K
\end{aligned}
$$

and now

$$
\begin{aligned}
\nu^{\prime} & =\frac{2 \pi}{\lambda}\left(\frac{a}{z_{2}}\right) \sqrt{\left(u^{\prime}\right)^{2}+\left(v^{\prime}\right)^{2}} \\
\phi^{\prime} & =\arctan \frac{v^{\prime}}{u^{\prime}} .
\end{aligned}
$$

The corresponding cartesian coordinate relationships are as follows,

$$
z^{\prime}=z+2\left(\frac{z_{2}}{a}\right)^{2} H \quad u^{\prime}=u+\left(\frac{z_{2}}{a}\right) L \quad v^{\prime}=v+\left(\frac{z_{2}}{a}\right) K
$$

Solving for $U^{\prime}\left(P^{\prime}\right)$ we find,

$$
\begin{aligned}
U^{\prime}\left(P^{\prime}\right) & =-\frac{i}{\lambda} \frac{a^{2} A}{z_{2}^{2}} e^{i\left(z_{2} / a\right)^{2} \zeta^{\prime}} \int_{0}^{1} \int_{0}^{2 \pi} \exp \left\{i\left[k \Phi^{\prime}-\nu^{\prime} \rho \cos \left(\theta-\phi^{\prime}\right)-\frac{1}{2} \zeta^{\prime} \rho^{2}\right]\right\} \rho d \rho d \theta \\
& =-\frac{i}{\lambda} \frac{a^{2} A}{z_{2}^{2}} e^{i\left(z_{2} / a\right)^{2} \zeta^{\prime}} \int_{0}^{1} \int_{0}^{2 \pi} \exp \{i[f(\zeta, \nu, \phi ; \rho, \theta)+k N]\} \rho d \rho d \theta \\
& =U(P) e^{i k N} e^{i\left(z_{2} / a\right)^{2}\left(\zeta^{\prime}-\zeta\right)}
\end{aligned}
$$


Eq.(3.23) is the displacement theorem expressed in terms of field. Note that $U^{\prime}\left(P^{\prime}\right)$ and $U(P)$ are only different by a constant phase term, $e^{i k\left(N+z^{\prime}-z\right)}$, and that in Born \& Wolf [1], the displacement theorem is expressed in terms of intensity, i.e. $\left|U^{\prime}\left(P^{\prime}\right)\right|^{2}=$ $|U(P)|^{2}$.

\section{PSF for distortion and defocus}

Using the displacement theorem, the PSFs of distortion and defocus can now be obtained from the unaberrated PSF, i.e. $\Phi=0$ [1]. With $\Phi=0$, Eq.(3.17) now becomes,

$$
\Phi^{\prime}=H \rho^{2}+K \rho \sin \theta+L \rho \cos \theta+N,
$$

which is purely distortion and defocus. Rewriting Eq.(3.21) in terms of the cartesian coordinate $(x, y)$ of the exit-pupil, we have

$U^{\prime}\left(P^{\prime}\right)=-\frac{i}{\lambda} \frac{a^{2} A}{z_{2}^{2}} e^{i\left(z_{2} / a\right)^{2} \zeta^{\prime}} \iint_{\mathbf{P}(x, y)} e^{i k \Phi^{\prime}} \exp \left\{-i \frac{2 \pi}{\lambda z_{2}}\left[x u^{\prime}+y v^{\prime}+\frac{\pi}{\lambda}\left(\frac{a}{z_{2}}\right)^{2} z^{\prime}\left(x^{2}+y^{2}\right)\right]\right\} d x d y$

Using the result of Eq.(3.23), with $U(P)$ of Eq.(3.12) being substituted in, note that now $\Phi=0$ for unaberrated case,

$$
\begin{aligned}
U^{\prime}\left(P^{\prime}\right) & =-\frac{i}{\lambda} \frac{a^{2} A}{z_{2}^{2}} e^{i\left(z_{2} / a\right)^{2} \zeta^{\prime}} \times \int_{0}^{1} \int_{0}^{2 \pi} \exp \left\{-i\left[\nu \rho \cos (\theta-\phi)+\frac{1}{2} \zeta \rho^{2}\right]\right\} \rho d \rho d \theta \times e^{i k N} \\
& =-\frac{i}{\lambda} \frac{a^{2} A}{z_{2}^{2}} e^{i\left(z_{2} / a\right)^{2} \zeta^{\prime}} \times 2 \pi \int_{0}^{1} J_{o}(\nu \rho) e^{-i \frac{1}{2} \zeta \rho^{2}} \rho d \rho \quad \times \quad e^{i k N}
\end{aligned}
$$

Equating Eq.(3.25) and Eq.(3.27), it is found that

$$
\begin{aligned}
U^{\prime}\left(P^{\prime}\right) & =-\frac{i}{\lambda} \frac{a^{2} A}{z_{2}^{2}} e^{i\left(z_{2} / a\right)^{2} \zeta^{\prime}} \times \iint_{\mathbf{P}(x, y)} e^{i k \Phi^{\prime}} \exp \left\{-i \frac{2 \pi}{\lambda z_{2}}\left[x u^{\prime}+y v^{\prime}+\frac{\pi}{\lambda}\left(\frac{a}{z_{2}}\right)^{2} z^{\prime}\left(x^{2}+y^{2}\right)\right]\right\} d x d y \\
& =-\frac{i}{\lambda} \frac{a^{2} A}{z_{2}^{2}} e^{i\left(z_{2} / a\right)^{2} \zeta^{\prime}} \times 2 \pi \int_{0}^{1} J_{o}(\nu \rho) e^{-i \frac{1}{2} \zeta \rho^{2}} \rho d \rho \quad \times \quad e^{i k N}
\end{aligned}
$$


As shown in Eq.(3.7), the effect of the aberration of interest is examined at $z^{\prime}=0$ in this case, i.e.,

$$
\begin{aligned}
U^{\prime}\left(u^{\prime}, v^{\prime}, z^{\prime}=0\right) & =-\frac{i}{\lambda} \frac{a^{2} A}{z_{2}^{2}} \times \iint_{\mathbf{P}(x, y)} e^{i k \Phi^{\prime}} \exp \left\{-i \frac{2 \pi}{\lambda z_{2}}\left[x u^{\prime}+y v^{\prime}\right]\right\} d x d y \\
& =-\frac{i}{\lambda} \frac{a^{2} A}{z_{2}^{2}} \times 2 \pi\left[\int_{0}^{1} J_{o}(\nu \rho) e^{-i \frac{1}{2} \zeta \rho^{2}} \rho d \rho\right] e^{i k N}
\end{aligned}
$$

One sees from Eq.(3.29), the Fourier transform of a defocused and/or distorted pupil can be numerically evaluated by calculating the integral,

$$
\int_{0}^{1} J_{o}(\nu \rho) e^{-i \frac{1}{2} \zeta \rho^{2}} \rho d \rho=e^{-i \zeta / 4} \sqrt{\frac{2 \pi}{\zeta}} \sum_{s=0}^{\infty}(i)^{s}(2 s+1) J_{s+\frac{1}{2}}(\zeta / 4) \frac{J_{2 s+1}(\nu)}{\nu}
$$

The procedure in the software used for numerically evaluating the PSF of the aberrated pupil is outlined in the flow diagram Fig. 3.2, basically utilizing the following equality:

$$
\begin{aligned}
\mathbf{G}_{\text {distort }}\left(u^{\prime}, v^{\prime}\right) & \equiv \iint_{\mathbf{P}(x, y)} e^{i k \Phi^{\prime}} \exp \left\{-i \frac{2 \pi}{\lambda z_{2}}\left[x u^{\prime}+y v^{\prime}\right]\right\} d x d y \\
& =2 \pi\left[e^{-i \zeta / 4} \sqrt{\frac{2 \pi}{\zeta}} \sum_{s=0}^{\infty}(i)^{s}(2 s+1) J_{s+\frac{1}{2}}(\zeta / 4) \frac{J_{2 s+1}(\nu)}{\nu}\right] e^{i k N}
\end{aligned}
$$

where

$$
\begin{array}{rlrl}
\Phi^{\prime} & =H \rho^{2}+K \rho \sin \theta+L \rho \cos \theta+N & & \\
z & =-2\left(\frac{z_{2}}{a}\right)^{2} H & & \\
u & =u^{\prime}-\left(\frac{z_{2}}{a}\right) L & \nu=\frac{2 \pi}{\lambda}\left(\frac{a}{z_{2}}\right)^{2} z \\
v & =v^{\prime}-\left(\frac{z_{2}}{a}\right) K & \nu=\frac{2 \pi}{\lambda}\left(\frac{a}{z_{2}}\right) \sqrt{u^{2}+v^{2}}
\end{array}
$$




\section{Summary on distortion \& defocus}

Expressing distortion and defocus in terms of the Zernike circle polynomials [48], i.e.

$$
\Phi_{\text {distort }}^{\prime}\left(u^{\prime}, v^{\prime}\right)=A_{\text {distort }} \rho \cos \theta+A_{\text {defocus }}\left(2 \rho^{2}-1\right)
$$

and comparing with Eq.(3.24), one finds

$$
\left\{\begin{array}{l}
H=2 A_{\text {defocus }} \quad ; \quad N=-A_{\text {defocus }} \\
L=A_{\text {distort }} \quad ; \quad K=0
\end{array}\right\}
$$

i.e.,

$$
\Phi^{\prime}=\left(2 A_{\text {defocus }}\right) \rho^{2}+\left(A_{\text {distort }}\right) \rho \cos \theta+\left(-A_{\text {defocus }}\right)
$$

and the corresponding PSF (up to a scaling constant) is as described in Eq.(3.31), and

$$
\begin{aligned}
& z=-2\left(\frac{z_{2}}{a}\right)^{2}\left(2 A_{\text {defocus }}\right) \\
& u=u^{\prime}-\left(\frac{z_{2}}{a}\right)\left(A_{\text {distort }}\right) \quad \zeta=\frac{2 \pi}{\lambda}\left(\frac{a}{z_{2}}\right)^{2} z \\
& v=v^{\prime} \quad \nu=\frac{2 \pi}{\lambda}\left(\frac{a}{z_{2}}\right) \sqrt{u^{2}+v^{2}}
\end{aligned}
$$

Note that here

$$
\left\{\begin{array}{l}
u^{\prime}=u_{i}+M \xi \\
v^{\prime}=v_{i}+M \eta
\end{array} \quad i=1,2\right.
$$

as in Eq.(3.7). 


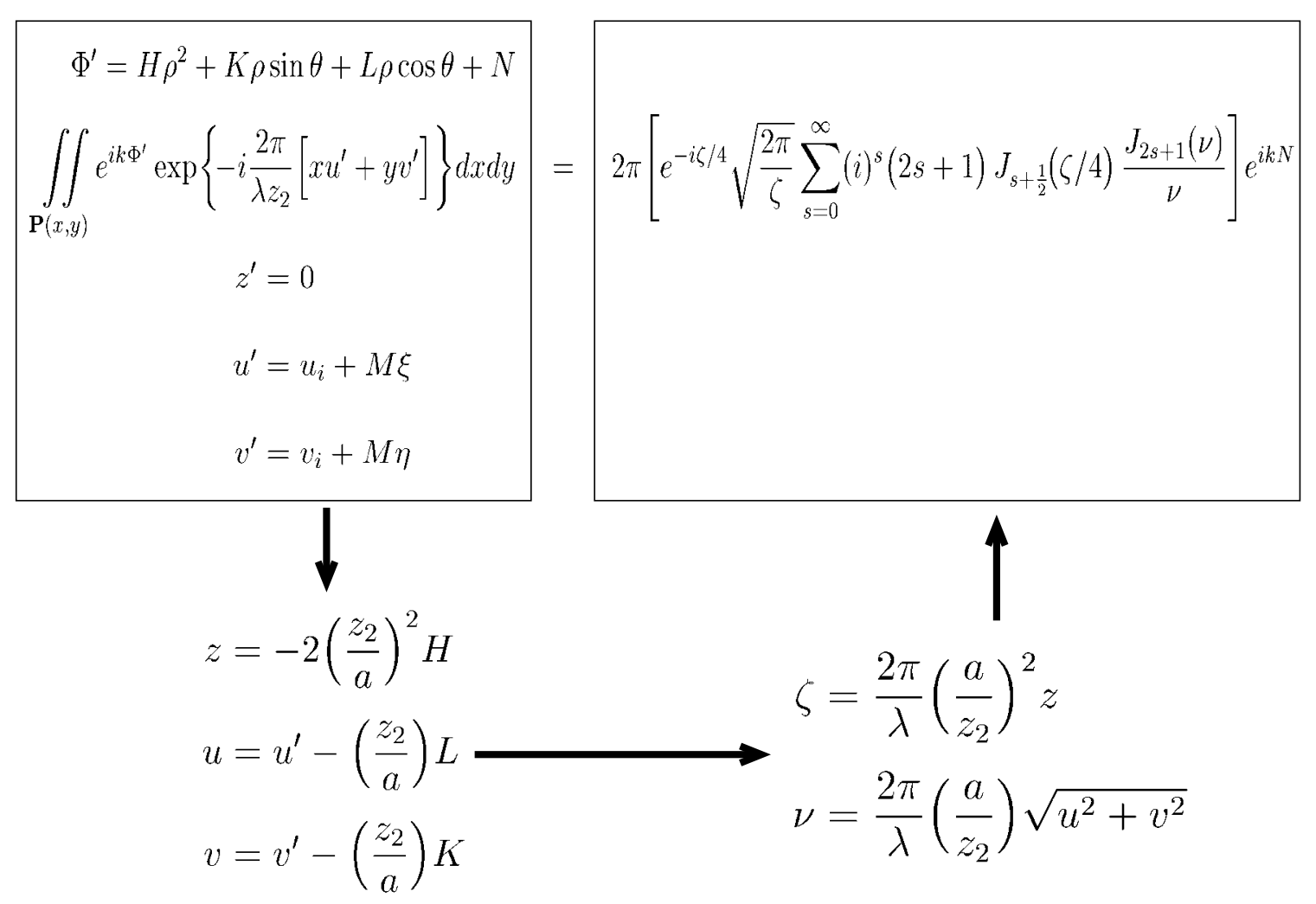

Figure 3.2: Procedure for the calculation of defocus/distortion PSF. 


\subsubsection{Astigmatism}

Next we consider the lower-order aberration of astigmatism, defined as

$$
\Phi_{\text {astig }}=A_{\text {astig }} \rho^{2} \cos 2 \theta
$$

where $A_{\text {astig }}$ is the aberration magnitude, usually of the order of wavelength $\lambda$.

In this case, the PSF (up to a scaling constant) produced by the aberrated pupil can be written as

$$
\mathbf{G}_{a s t i g}\left(u^{\prime}, v^{\prime}\right)=\iint_{\mathbf{P}(x, y)} e^{i k \Phi_{a s t i g}} \exp \left\{-i \frac{2 \pi}{\lambda z_{2}}\left[x u^{\prime}+y v^{\prime}\right]\right\} d x d y
$$

Following the derivation in Ref. [49], the above integral involving the aberrated pupil can be expressed in terms of a series of Bessel functions. Note that the signs of the imaginary parts in the equation are inverted to conform with the notation used here.

$$
\begin{aligned}
\mathbf{G}_{\text {astig }}\left(u^{\prime}, v^{\prime}\right) \cong \frac{2 \pi}{\nu}[ & J_{1}(\nu)-i \beta \cos 2 \phi J_{3}(\nu) \\
& -\frac{\beta^{2}}{2 \cdot 2 !}\left\{\frac{1}{3} J_{1}(\nu)-\frac{1}{2} J_{3}(\nu)+\frac{1}{6} J_{5}(\nu)+\cos 4 \phi J_{5}(\nu)\right\} \\
& -\frac{i \beta^{3}}{2^{2} \cdot 3 !}\left\{-3 \cos 2 \phi\left(\frac{3}{5} J_{3}(\nu)-\frac{1}{3} J_{5}(\nu)+\frac{1}{15} J_{7}(\nu)\right)-\cos 6 \phi J_{7}(\nu)\right\} \\
& +\frac{\beta^{4}}{2^{3} \cdot 4 !}\left\{3\left(\frac{1}{5} J_{1}(\nu)-\frac{2}{5} J_{3}(\nu)+\frac{2}{7} J_{5}(\nu)-\frac{1}{10} J_{7}(\nu)+\frac{1}{70} J_{9}(\nu)\right)\right. \\
& \left.\left.\quad+4 \cos 4 \phi\left(\frac{5}{7} J_{5}(\nu)-\frac{1}{4} J_{7}(\nu)+\frac{1}{28} J_{9}(\nu)\right)+\cos 8 \phi J_{9}(\nu)\right\}\right] .
\end{aligned}
$$

where

$$
\begin{aligned}
\beta & =k A_{\text {astig }}=\frac{2 \pi}{\lambda} A_{\text {astig }} & \\
\nu^{\prime} & =\frac{2 \pi}{\lambda}\left(\frac{a}{z_{2}}\right) \sqrt{\left(u^{\prime}\right)^{2}+\left(v^{\prime}\right)^{2}} & \equiv \nu \\
\phi^{\prime} & =\arctan \frac{v^{\prime}}{u^{\prime}} & \equiv \phi
\end{aligned}
$$


Note that here we have dropped the primes for $\nu^{\prime}$ and $\phi^{\prime}$ for ease of notation, and that with respect to the image-plane mutual intensity expression in Eq.(3.7),

$$
\left\{\begin{array}{l}
u^{\prime}=u_{i}+M \xi \\
v^{\prime}=v_{i}+M \eta
\end{array} \quad i=1,2\right.
$$

Therefore,

$$
\begin{aligned}
\nu & =\frac{2 \pi}{\lambda}\left(\frac{a}{z_{2}}\right) \sqrt{\left(u_{i}+M \xi\right)^{2}+\left(v_{i}+M \eta\right)^{2}} \\
\phi & =\arctan \frac{v_{i}+M \eta}{u_{i}+M \xi}
\end{aligned}
$$

where $a$ is the condenser exit-pupil radius.

\subsubsection{Coma}

Finally, we consider another lower-order aberration, namely coma. Coma is defined as

$$
\Phi_{\text {coma }}=A_{\text {coma }}\left(3 \rho^{3}-2 \rho\right) \cos \theta
$$

where $A_{\text {coma }}$ is the aberration magnitude.

The resultant PSF (up to a scaling constant) is

$$
\mathbf{G}_{\text {coma }}\left(u^{\prime}, v^{\prime}\right)=\iint_{\mathbf{P}(x, y)} e^{i k \Phi_{\text {coma }}} \exp \left\{-i \frac{2 \pi}{\lambda z_{2}}\left[x u^{\prime}+y v^{\prime}\right]\right\} d x d y
$$

The above integral can be expressed as a series of Bessel functions as follows,

$$
\begin{aligned}
\mathbf{G}_{\text {coma }}\left(u^{\prime}, v^{\prime}\right) \cong \frac{2 \pi}{\nu} & {\left[J_{1}(\nu)-\beta \cos \phi J_{4}(\nu)\right.} \\
& -\frac{\beta^{2}}{2 \cdot 2 !}\left\{\frac{1}{4} J_{1}(\nu)-\frac{1}{20} J_{3}(\nu)+\frac{1}{4} J_{5}(\nu)-\frac{9}{20} J_{7}(\nu)-\cos 2 \phi\left(\frac{2}{5} J_{3}(\nu)+\frac{3}{5} J_{7}(\nu)\right)\right\} \\
& -\frac{\beta^{3}}{2^{2} \cdot 3 !}\left\{3 \cos \phi\left(\frac{1}{15} J_{2}(\nu)-\frac{44}{105} J_{4}(\nu)+\frac{9}{70} J_{6}(\nu)-\frac{6}{35} J_{8}+\frac{3}{14} J_{10}(\nu)\right)\right. \\
& \left.\left.\quad-\cos 3 \phi\left(\frac{8}{35} J_{4}(\nu)-\frac{9}{20} J_{6}(\nu)-\frac{9}{28} J_{10}(\nu)\right)\right\}\right] .
\end{aligned}
$$


where

$$
\begin{aligned}
\beta & =k A_{\text {coma }}=\frac{2 \pi}{\lambda} A_{\text {coma }} & \\
\nu^{\prime} & =\frac{2 \pi}{\lambda}\left(\frac{a}{z_{2}}\right) \sqrt{\left(u^{\prime}\right)^{2}+\left(v^{\prime}\right)^{2}} & \equiv \nu \\
\phi^{\prime} & =\arctan \frac{v^{\prime}}{u^{\prime}} & \equiv \phi
\end{aligned}
$$

Again here we have dropped the primes for $\nu^{\prime}$ and $\phi^{\prime}$ for ease of notation and that again with respect to the image-plane mutual intensity expression in Eq.(3.7),

$$
\left\{\begin{array}{l}
u^{\prime}=u_{i}+M \xi \\
v^{\prime}=v_{i}+M \eta
\end{array} \quad i=1,2\right.
$$

Therefore,

$$
\begin{aligned}
\nu & =\frac{2 \pi}{\lambda}\left(\frac{a}{z_{2}}\right) \sqrt{\left(u_{i}+M \xi\right)^{2}+\left(v_{i}+M \eta\right)^{2}} \\
\phi & =\arctan \frac{v_{i}+M \eta}{u_{i}+M \xi}
\end{aligned}
$$

\subsection{Numerical evaluation results}

Using the object-image coherence relation derived in Sec. 3.3, i.e. Eq.(3.7), the mutual intensity at the image plane of the condenser can now be determined numerically by incorporating the appropriate PSFs [i.e. $\mathbf{G}\left(u^{\prime}, v^{\prime}\right)$ ] given in Sec. 3.4. To test the validity of the numerical evaluation, a large $(1.6 \mathrm{~mm} \times 1.6 \mathrm{~mm})$ uniform intensity incoherent source is used to illuminate the condenser. Unlike the undulator source, this large square incoherent source can be shown to satisfy the Zernike approximation. The resultant intensity and coherence distributions with various pupil aberrations are shown in Fig. 3.3. As expected by the Zernike approximation, the various aberrations have negligible effect on the spatial coherence distributions, which are all essentially Airy patterns with first null radius of $2.8 \mu \mathrm{m}$. The intensity distributions are all relatively uniform, again as expected by the Zernike approximation. In the case of coma, the intensity distribution is shifted as the center of mass of the modulus square of the coma PSF is off-centered. 

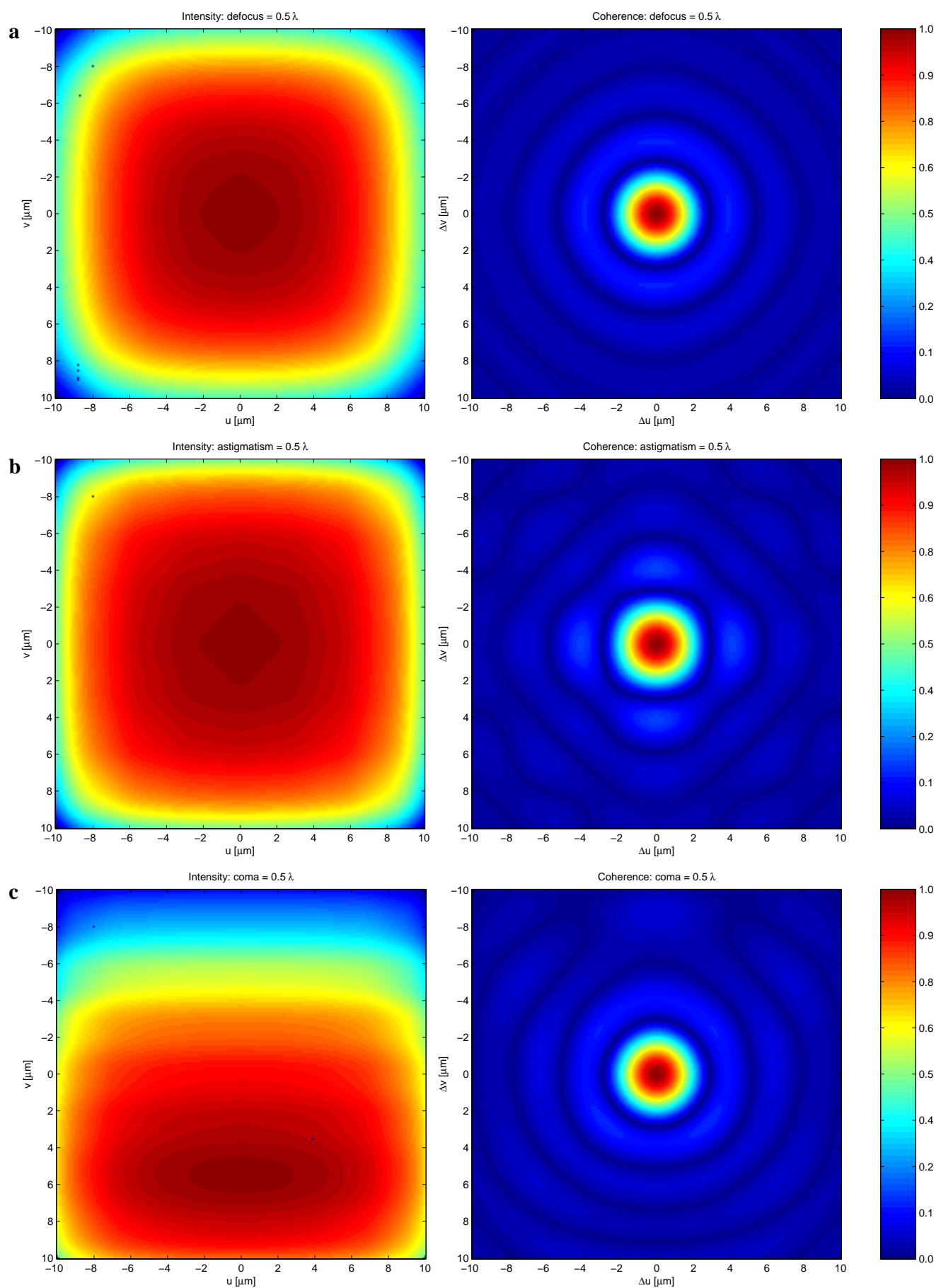

Figure 3.3: Simulation results for a large $(1.6 \mathrm{~mm} \times 1.6 \mathrm{~mm})$ uniform source. Intensity (left column) and coherence (right column) distributions resulting from the various pupil aberrations (a defocus, $\mathbf{b}$ astigmatism, $\mathbf{c}$ coma). The coherence distributions are all essentially Airy patterns as predicted by the Zernike approximation. 
Next, an actual undulator source having a size that does not satisfy the Zernike approximation is studied. The intensity distribution of this incoherent undulator source is Gaussian with $\left(\sigma_{x}, \sigma_{y}\right)=(260 \mu m, 16 \mu m)$, given by the undulator beam size at the exit-plane. First, an aberration-free condenser is assumed in order to see the effect of smaller source size. Figure 3.4 shows the failure of Zernike approximation for small sources, as the coherence distribution deviates significantly from an Airy pattern.
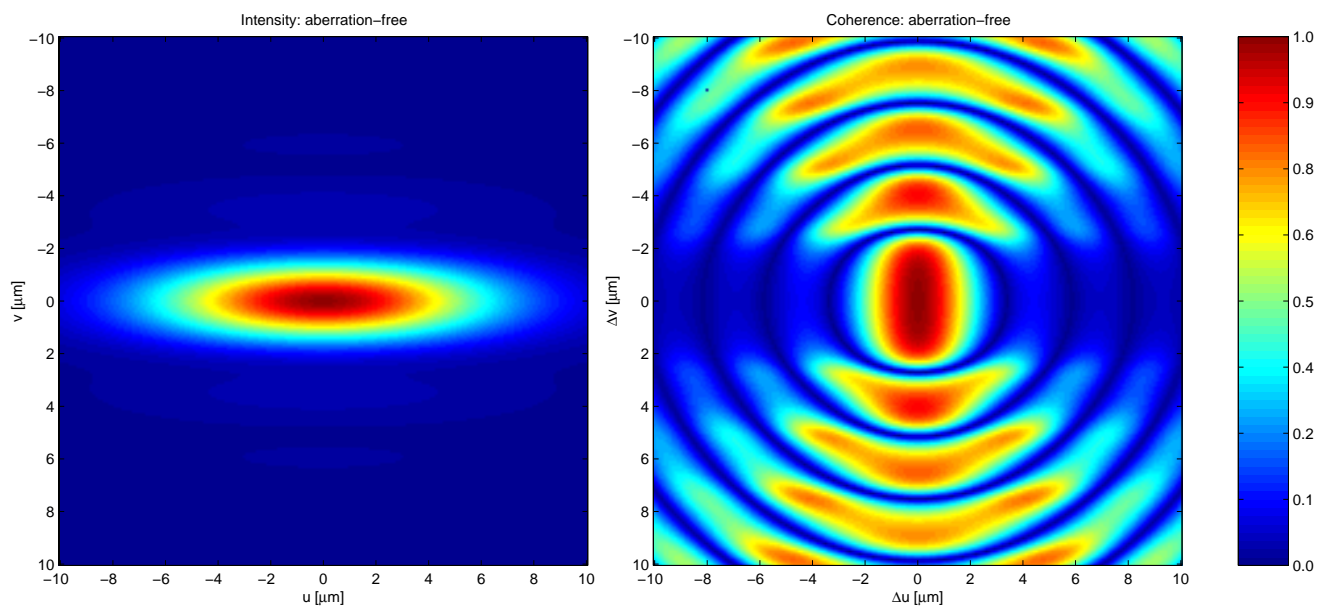

Figure 3.4: Simulation results: Intensity and coherence distribution at the condenser image-plane resulting from the un-aberrated condenser pupil and the Gaussian-shaped incoherent source of $\left(\sigma_{x}, \sigma_{y}\right)=(260 \mu m, 16 \mu m)$.

It has been shown above that the size of the incoherent source has affected the coherence distribution at the image plane and the apodized pupil function is not the sole determining factor. Next, the effect of pupil aberrations on the spatial coherence distribution is investigated. Several low order aberrations will be assumed in the condenser to demonstrate this effect. The PSFs needed by Eq.(3.7) are again given in Sec. 3.4. Figure 3.5 shows that in the case of smaller source size, the effect of condenser aberrations on spatial coherence cannot be ignored. With 0.5 waves of defocus in the condenser, the intensity and coherence distributions at the condenser-image plane is shown in Fig. 3.5(a). Figures 3.5(b) and (c) show the distributions under 0.5 waves of astigmatism and coma, respectively. For the cases of defocus and astigmatism, the high vertical coherence shown in Fig. 3.5(a) and (b) can be explained by the small 
vertical source size. However, in general the two dimensional coherence distributions cannot be treated separately in terms of vertical and horizontal directions. As demonstrated in the case of coma, shown in Fig. 3.5(c), the two dimensional structure of coma dominates the spatial coherence distribution in the condenser image-plane and the coherence distribution cannot be explained simply by the vertical and horizontal source size. It is shown that numerical spatial coherence propagation based on the Huygens-Fresnel principle is required to correctly predict the coherence distribution at the condenser image-plane.

Note again that the coherence distribution is not simply a function of the difference of the coordinates, instead, it is a function of the four individual coordinates, i.e. $\left(u_{1}, v_{1}, u_{2}, v_{2}\right)$. Therefore, when showing the coherence distribution, one of the coordinates is fixed at the origin, i.e. $\left(u_{1}, v_{1}\right)=(0,0)$, and the coherence distribution is obtained as the correlation factor $\left|\boldsymbol{\mu}_{12}\right|$ between various points $\left(u_{2}, v_{2}\right)$ and the origin $(0,0)$.

Note that for a condenser system illuminated by a point source (i.e. a coherent source), the resultant intensity and coherence distributions at the image plane of the condenser can also be obtained with this numerical evaluation by employing a Dirac- $\delta$-function as the source. The results for 0.5 waves of defocus, astigmatism and coma, respectively, are shown in Fig. 3.6. The intensity distribution indeed shows the modulus square of the PSF of the respective lens pupil and the resultant coherence distribution is 1 at all positions since it is coherently illuminated. 

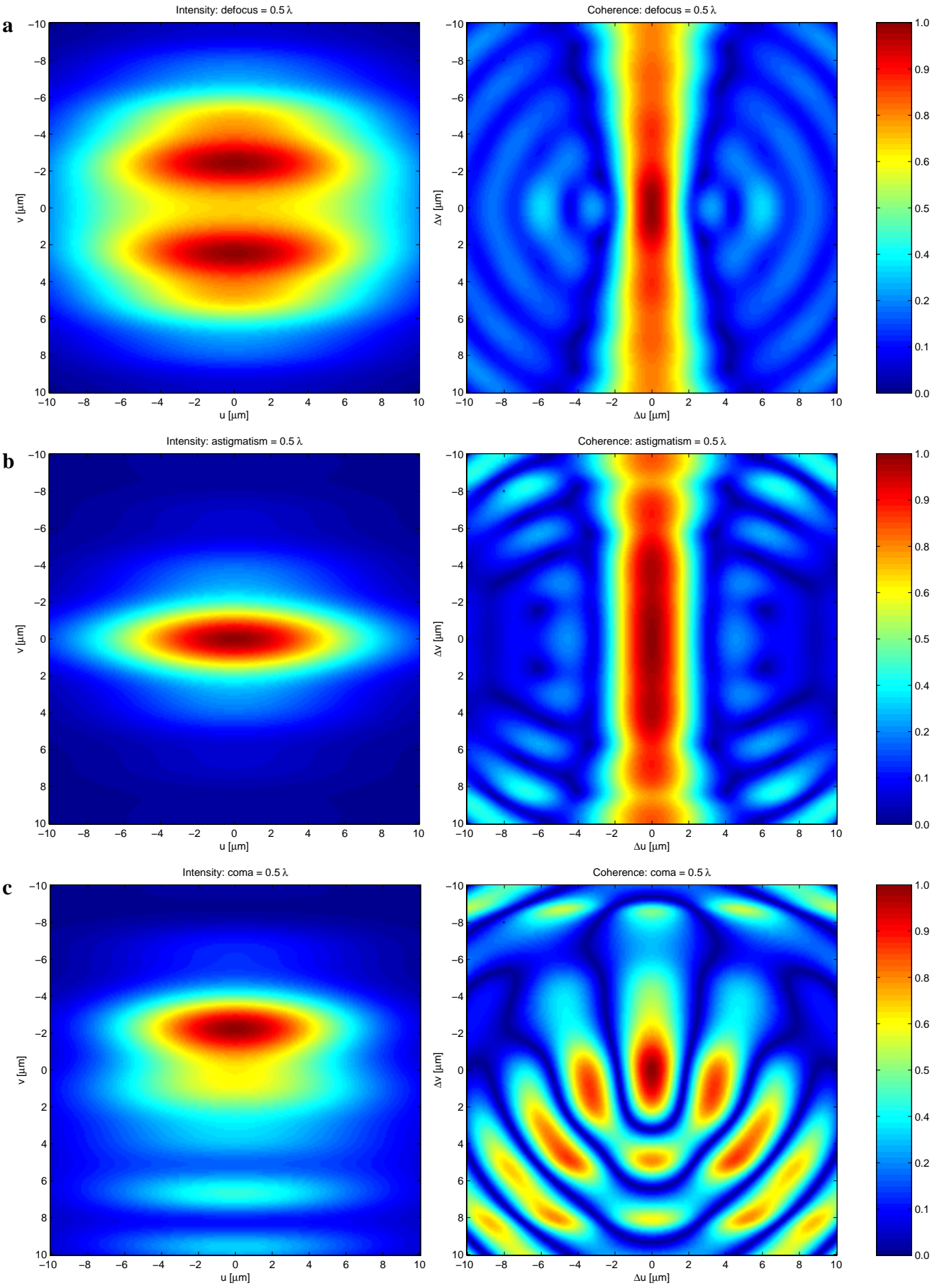

Figure 3.5: Simulation results that show the effect of aberrations for the Gaussianshaped incoherent source: Intensity (left column) and coherence (right column) distribution at resulting from the various aberrations (a defocus, $\mathbf{b}$ astigmatism, $\mathbf{c}$ coma). 
a

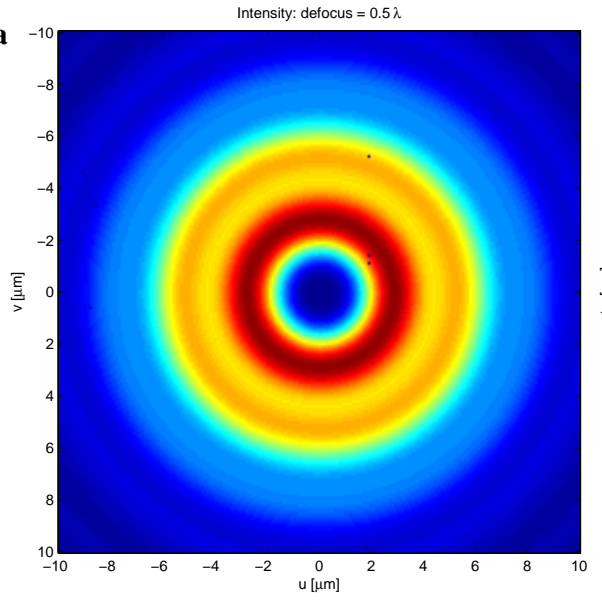

Intensity: astigmatism $=0.5 \lambda$

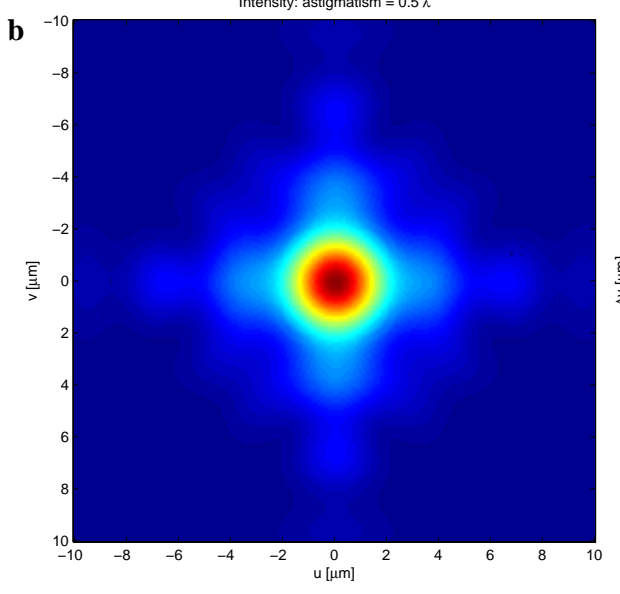

Intensity: coma $=0.5 \lambda$

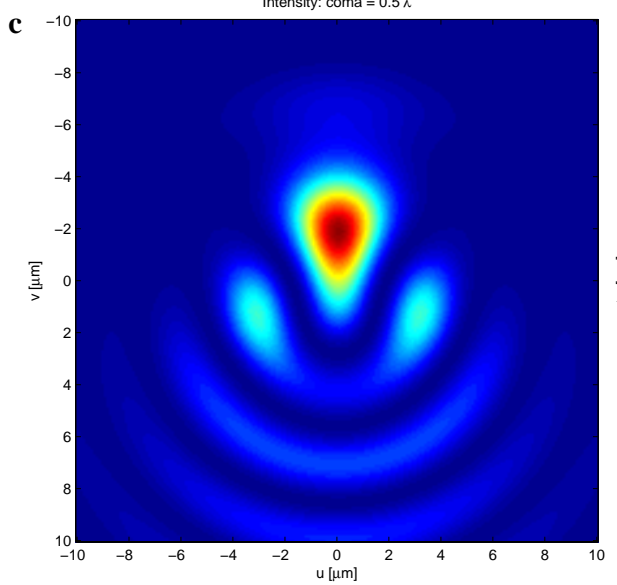

Coherence: defocus $=0.5 \lambda$

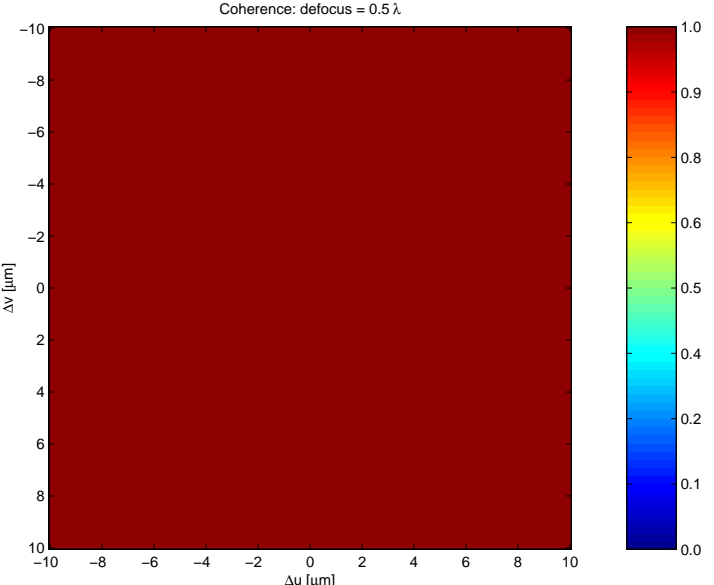

Coherence: astigmatism $=0.5 \lambda$

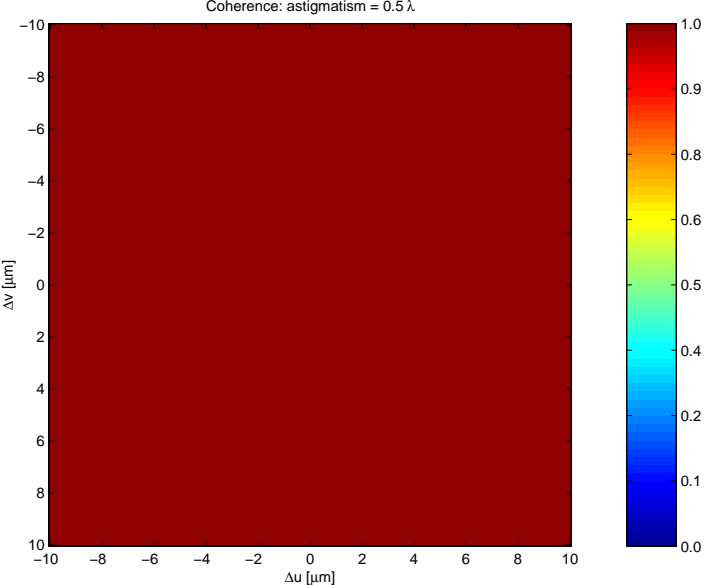

Coherence: coma $=0.5 \lambda$

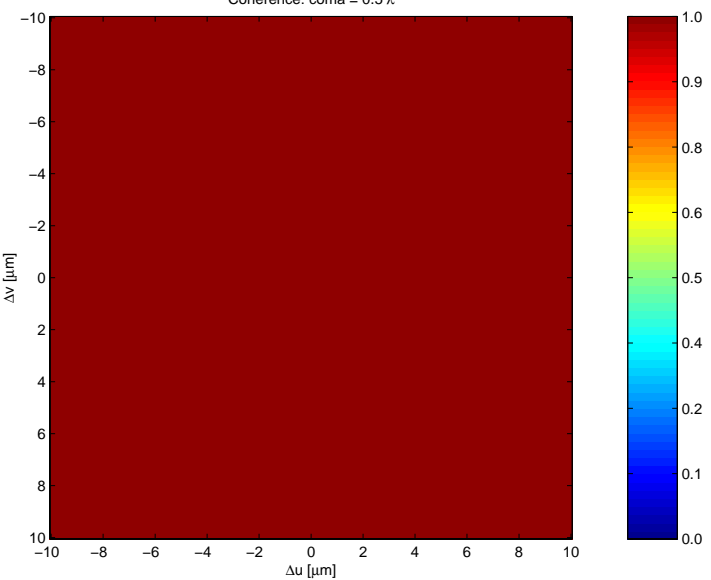

Figure 3.6: Simulation results using a point source (coherent source): Intensity (left column) and coherence (right column) distributions resulting from the various pupil aberrations (a defocus, b astigmatism, c coma). The coherence distribution is a constant 1 , which shows that the field is fully coherent as expected. 


\subsection{Conclusion}

The results obtained in this chapter are valid for any condenser systems that re-image the incoherent source to its conjugate plane, regardless of the Zernike approximation. We have demonstrated that for a condenser system employing an EUV undulator as the radiation source, the commonly used Zernike approximation is violated and numerical spatial coherence propagation based on Huygens-Fresnel principle is required. In fact, for any condenser system that re-images an incoherent source, the spatial coherence distribution at the image plane, in general, depends both on the complex pupil function, and on the intensity distribution of the incoherent source. Using the Zernike approximation requires caution and the validity of Zernike approximation has to be verified before application. Also shown is that pupil aberrations pose significant effect on the spatial coherence distributions at the condenser image-plane when Zernike approximation fails. 


\section{Chapter 4}

\section{Diffractive optical elements based on Fourier optical techniques:}

\section{A new class of short wavelength optical elements}

A diffractive optical element, based on Fourier optical techniques, for use in extreme ultraviolet/soft x-ray experiments, has been fabricated and demonstrated. This diffractive optical element, when illuminated by a uniform plane wave, will produce two symmetric off-axis first order foci suitable for interferometric experiments. The efficiency of this optical element, and its use in an EUV interferometer, are presented. Its use opens a new era in the use of sophisticated optical techniques at short wavelengths.

\subsection{Introduction}

Coherent extreme ultraviolet (EUV) and soft x-ray (SXR) radiation [35] facilitates phase-sensitive techniques that provide new opportunities in various fields, e.g. biological imaging, material characterization, and nanotechnology. However, challenges are presented in that very limited optical elements are available at these wavelengths. Most experiments either utilize low efficiency diffractive optics such 
as Fresnel zoneplates, or glancing incidence reflection mirrors and normal incidence multi-layer mirrors which result in restrictive off-axis optical systems and limited spectral region, respectively. No appropriate materials exist for lenses and prisms due to high absorption. Therefore, devising novel optical elements that can effectively and efficiently achieve wavefront shaping is of crucial importance for researches conducted at EUV/SXR wavelengths. Here, Fourier optical techniques are introduced to accomplish the desired wavefront manipulation.

In our first example of these new techniques, we have designed and fabricated, based on Fourier optical techniques, a diffractive optical element which combines the functions of a grating and a zone-plate through a bit-wise XOR operation [11]. Using this compound diffractive optical element allows the efficiency and the contrast of the interferometer to be greatly increased. The application of this optical element in an EUV interferometer to directly determine the index of refraction at EUV wavelengths will be presented in Chapter 5. Similar activities are underway at soft x-ray wavelengths.

\subsection{XOR pattern}

This XOR diffractive optical element is obtained by combining a $50 \%$ duty-cycle binary intensity grating and a $50 \%$ duty-cycle intensity zoneplate. The binary grating and zoneplate are first pixelized, with each pixel being either 1 or 0 for transmission and absorption, respectively. As shown in Fig. 4.1, the two pixelized patterns are then overlapped and compared pixel by pixel to produce the resulting XOR pattern, i.e. at each pixel position, if the pixel values of the grating and zoneplate are the same (both 0's or both 1's), the value of the corresponding pixel on the XOR pattern is 0 . Otherwise, the value of the corresponding pixel on the XOR pattern is 1 .

For a $50 \%$ duty-cycle grating of period $d$, the transmitted intensity function is

$$
G(x, y)=\frac{1}{2}[1+\operatorname{sgn}(\cos \nu x)]
$$

where $\nu=2 \pi / d$. 
Similarly, for a $50 \%$ duty-cycle zoneplate of diameter $D$ and outermost zone-width $\Delta r$, the transmitted intensity function is [2]

$$
Z P(x, y)=\frac{1}{2}\left[1+\operatorname{sgn}\left(\cos \gamma r^{2}\right)\right]
$$

where $r=\sqrt{x^{2}+y^{2}}$ and

$$
\gamma=\frac{\pi}{\Delta r(D-\Delta r)} .
$$

Expand these two patterns in their Fourier series,

$$
G(x, y)=\sum_{m=-\infty}^{\infty} \frac{\sin (m \pi / 2)}{m \pi} e^{-j m \nu x}
$$

and

$$
Z P(x, y)=\sum_{n=-\infty}^{\infty} \frac{\sin (n \pi / 2)}{n \pi} e^{-j n \gamma r^{2}}
$$

Note that by comparing the Fourier series of a zoneplate to a lens, one finds that the zoneplate functions as multiple lenses with $n^{\text {th }}$ order focal length $f_{n}$ given by

$$
f_{n}=\frac{-\pi}{n \lambda \gamma}
$$

The XOR pattern of the combined grating and the zoneplate is obtained by

$$
\begin{aligned}
X O R(x, y)= & G(x, y)+Z P(x, y)-2 G(x, y) Z P(x, y) \\
= & \sum_{m=-\infty}^{\infty} \frac{\sin (m \pi / 2)}{m \pi} e^{-j m \nu x}+\sum_{n=-\infty}^{\infty} \frac{\sin (n \pi / 2)}{n \pi} e^{-j n \gamma r^{2}} \\
& -2\left[\frac{1}{2}+\sum_{\substack{m=-\infty \\
m \neq 0}}^{\infty} \frac{\sin (m \pi / 2)}{m \pi} e^{-j m \nu x}\right]\left[\frac{1}{2}+\sum_{\substack{n=-\infty \\
n \neq 0}}^{\infty} \frac{\sin (n \pi / 2)}{n \pi} e^{-j n \gamma r^{2}}\right] \\
= & \frac{1}{2}-2\left[\sum_{\substack{m=-\infty \\
m \neq 0}}^{\infty} \frac{\sin (m \pi / 2)}{m \pi} e^{-j m \nu x}\right]\left[\sum_{\substack{n=-\infty \\
n \neq 0}}^{\infty} \frac{\sin (n \pi / 2)}{n \pi} e^{-j n \gamma r^{2}}\right] .
\end{aligned}
$$

This combined diffractive element, when illuminated by a uniform wavefront, has the interesting property that it produces two symmetric off-axis focal spots, $(m, n)=$ 
$( \pm 1,1)$, at the back focal plane of the zoneplate. Note that both the grating and the zoneplate have to be of $50 \%$ duty-cycle for the on-axis focal spot to disappear, i.e. $m \neq 0$ and $n \neq 0$ in the summation. The separation of these two beam spots $x_{s}$ can be determined by multiplying the two exponentials in Eq.(4.5), completing the square for $x$-terms, thus resulting in

$$
x_{s}=\frac{2 \Delta r(D-\Delta r)}{d} \approx \frac{2 \Delta r D}{d} .
$$

Note that this separation is independent of wavelength $\lambda$. Thus as the wavelength is varied for spectral determinations of $\delta$ and $\beta$, the focal length (distance from the XOR pattern to the sample mask) varies, but the lateral separation of the two beam spots remains fixed. The invariance of the spot separation over wavelength allows the EUV interferometer to operate at different wavelengths without the need of changing the image-plane sample mask. This is a desirable property for EUV interferometers since the scale of the sample mask for EUV applications requires it to be micro/nanofabricated, thus immutable after being made. 


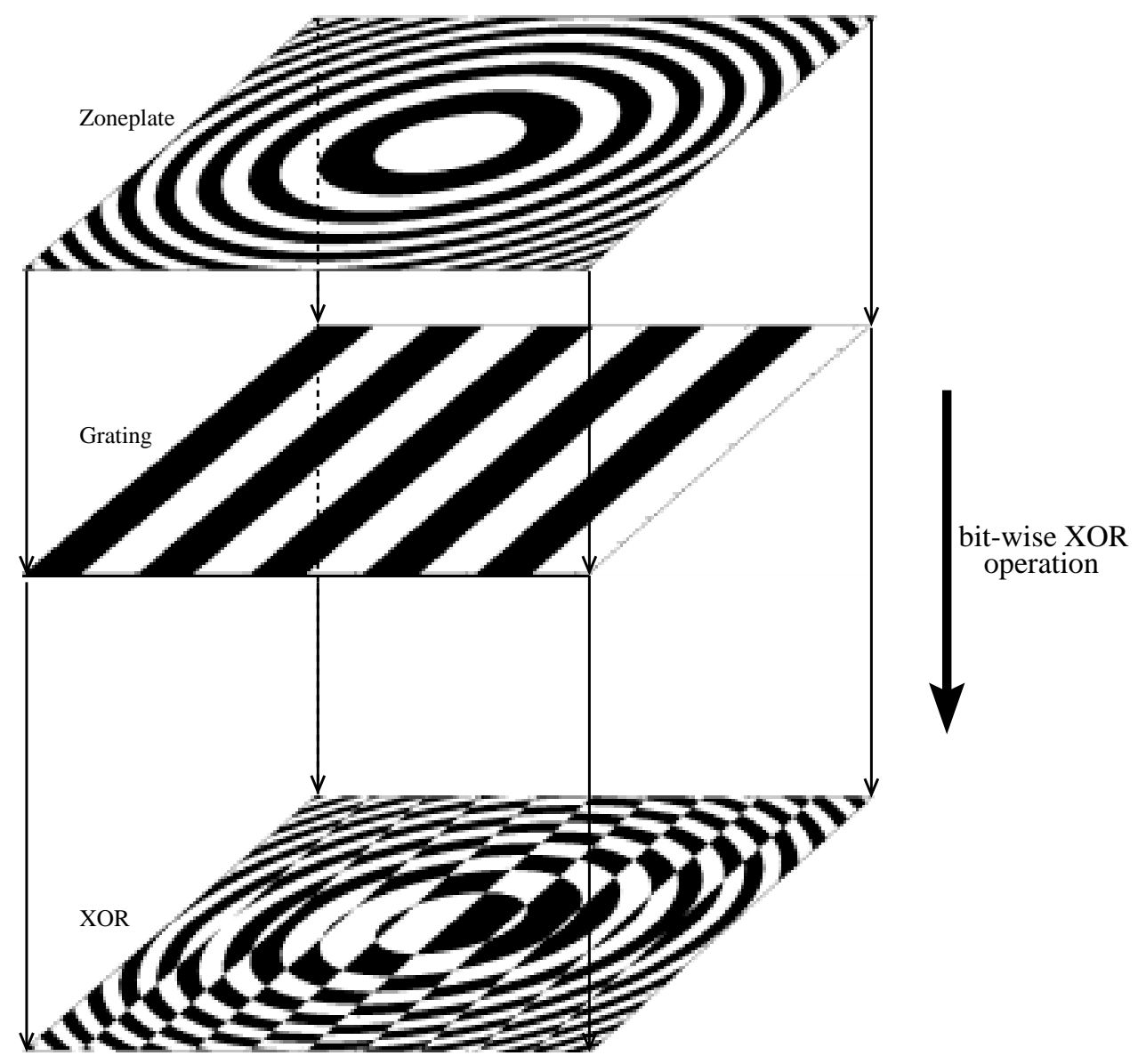

Figure 4.1: Bitwise XOR operation: The binary grating and zoneplate are first pixelized, with each pixel being either 1 or 0 for transmission and absorption, respectively. The two pixelized patterns are then overlapped and compared pixel by pixel to produce the resulting XOR pattern, i.e. at each pixel position, if the pixel values of the grating and zoneplate are the same (both 0's or both 1's), the value of the corresponding pixel on the XOR pattern is 0. Otherwise, the value of the corresponding pixel on the XOR pattern is 1 . 


\subsubsection{Simulation of the XOR pattern}

A computer simulation has been performed to see if these patterns produce the expected results. An XOR pattern of a grating (period $d=16 \mu m$ ) and a zoneplate (outermost zone-width $\Delta r=0.2 \mu \mathrm{m}$, diameter $D=400 \mu \mathrm{m}$ ) is produced, as shown in Fig. 4.2(a). This pattern is then Fresnel-propagated to the first order focal plane of the zoneplate and the resulting intensity distribution is shown in Fig. 4.2(b). As expected, only off-axis spots exist in this focal plane and the on-axis zeroth order focus is completely eliminated.
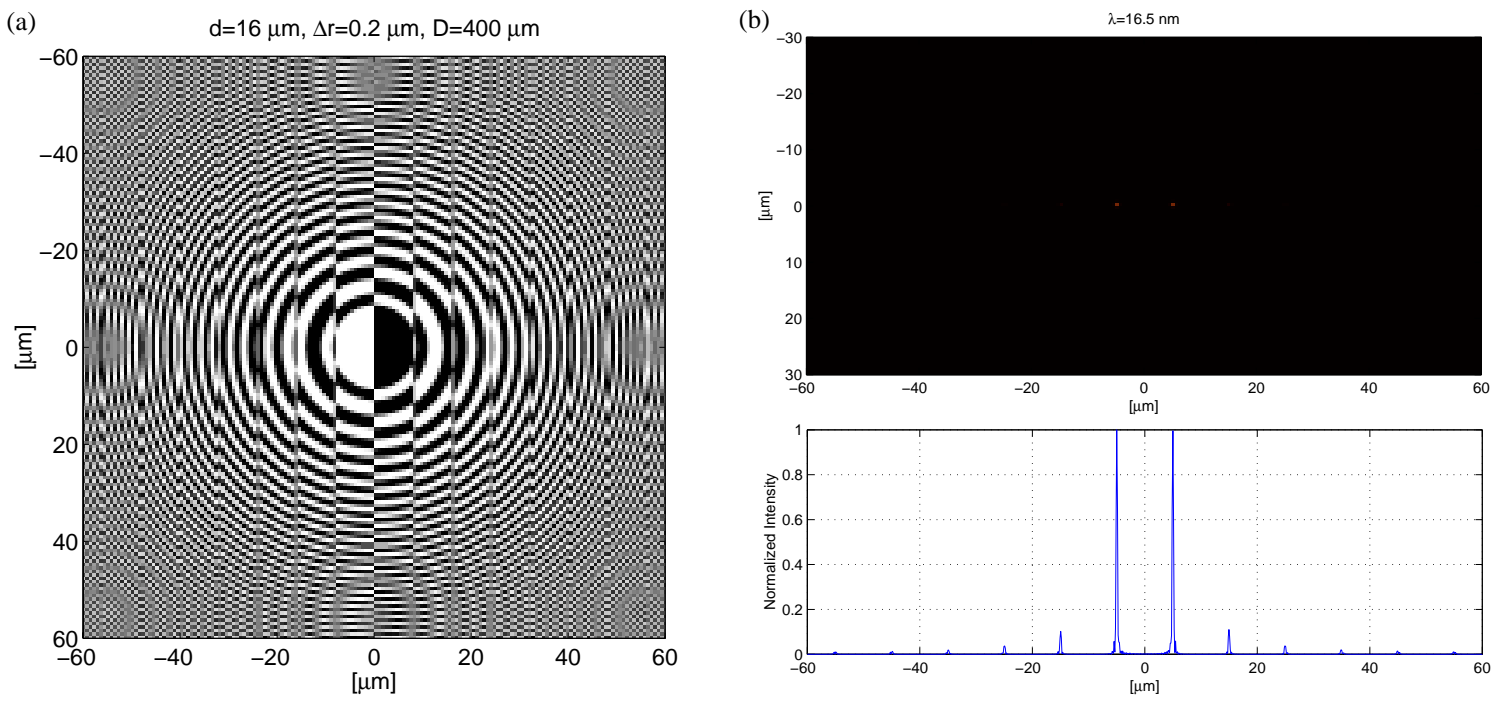

Figure 4.2: Computer simulation of the XOR pattern: The parameters used in this simulation are set equal to the actual fabricated element. The pattern in (a) is obtained by taking the "exclusive or (XOR)" of the binary grating and zone-plate. $4096 \times 4096$ pixels are used to generated this pattern. This pattern is then Fresnelpropagated in computer by one focal length and the resulting intensity distribution is shown in (b). A horizontal cross-section through the focal spots is also shown. The two symmetric off-axis first order foci is clearly visible in this simulation. The other two outer spots are caused by the third orders $(m= \pm 3)$ of the grating, with 9 times lower intensity. 


\subsection{Efficiency of the XOR pattern}

The XOR pattern, as expressed in Eq. 4.5, gives the efficiencies of the individual orders. First of all, we need to determine the overall transparent area of this XOR pattern. Since we know that the percent of transparent area on the grating and the zoneplate is $1 / 2$, we find that the overall transparent area of the XOR pattern to be $1 / 2+1 / 2-2(1 / 2)(1 / 2)=1 / 2$ from Eq. 4.5.

Next, we calculate the efficiency of individual orders from their relative strength. From Eq. 4.5, we have, for $m, n \neq 0$,

$$
\eta_{m, n}=\left\{\begin{array}{cc}
\frac{2^{2}(1 / m \pi)^{2}(1 / n \pi)^{2}}{(1 / 2)^{2}+2^{2}\left(2 \frac{1}{8}\right)\left(2 \frac{1}{8}\right)} \times \frac{1}{2}=\frac{4}{m^{2} n^{2} \pi^{4}} & \text { if } m, n \text { are both odd } \\
0 & \text { if } m \text { or } n \text { is even. }
\end{array}\right.
$$

where $\sum_{k=0}^{\infty} \frac{1}{(2 k+1)^{2}}=\frac{\pi^{2}}{8}$ is used in the calculation.

Another way to look at this is that we can think of this XOR pattern as a binary amplitude zoneplate, multiplied by a $\pi$-phase-shift grating which does not have any absorption. Therefore, the overall absorption of this XOR pattern is the same as that of a binary amplitude zoneplate, i.e. $1 / 2$ and the efficiency of its individual orders is given by multiplying the corresponding orders of the binary amplitude zoneplate and the $\pi$-phase-shift grating. The efficiency $\eta_{m}$ of a $50 \%$ duty-cycle $\pi$-phase-shift grating is

$$
\eta_{m}=\left\{\begin{array}{cl}
\frac{4}{m^{2} \pi^{2}} & \text { for } m= \pm 1, \pm 3, \cdots \\
0 & \text { for } m \text { is even }
\end{array}\right.
$$

And the efficiency $\eta_{n}$ of a binary amplitude zoneplate is

$$
\eta_{n}=\left\{\begin{array}{cl}
\frac{1}{n^{2} \pi^{2}} & \text { for } n= \pm 1, \pm 3, \cdots \\
0 & \text { for } n \text { is even }
\end{array}\right.
$$

By comparing Eq. 4.7 with Eq. 4.8 and Eq. 4.9, we indeed see that the efficiency of the individual orders of the XOR pattern, $\eta_{m, n}$, is given by $\eta_{m} \times \eta_{n}$, , i.e. the multiplication of the corresponding orders of the phase grating and amplitude zoneplate. 


\subsection{Visible light experiment}

A first XOR pattern, designed for proof-of-principle testing at visible wavelengths, is fabricated using e-beam lithography [22] in order to directly observe the intensity distribution at the back focal plane. The grating used in this visible version has a period of $5 \mu \mathrm{m}$, the zoneplate diameter is $5 \mathrm{~mm}$ and the outermost zone-width is $2 \mu \mathrm{m}$. A screen is put at its back plane, which is $15.8 \mathrm{~mm}$ away from this visible XOR pattern. A collimated He-Ne laser beam $(\lambda=633 \mathrm{~nm})$ is then used to illuminate this visible version XOR pattern and the resulting intensity distribution at the back focal plane is shown in Fig. 4.3(a). As expected, the two symmetric off-axis foci are directly observable and there is no on-axis focus presented. The separation between these two off-axis spots are measured to be $4 \mathrm{~mm}$, which agrees with the designed value. As a comparison, an "OR" pattern made from the same grating and zoneplate is also fabricated and shown in Fig. 4.4. The effect of combining the grating and zoneplate through an bit-wise OR operation is equivalent to that of placing them in tandem. Therefore, this OR pattern demonstrates the back focal plane intensity distribution of a traditional separate grating and zoneplate setup. Fig. 4.3(b) shows the resulting intensity distribution at the back focal plane of this OR pattern. Three foci are clearly observed, with the strongest focus on-axis and two weaker symmetric off-axis foci. The separation between the on-axis and the off-axis spots are measured to be $2 \mathrm{~mm}$, which again agrees with the designed value. 

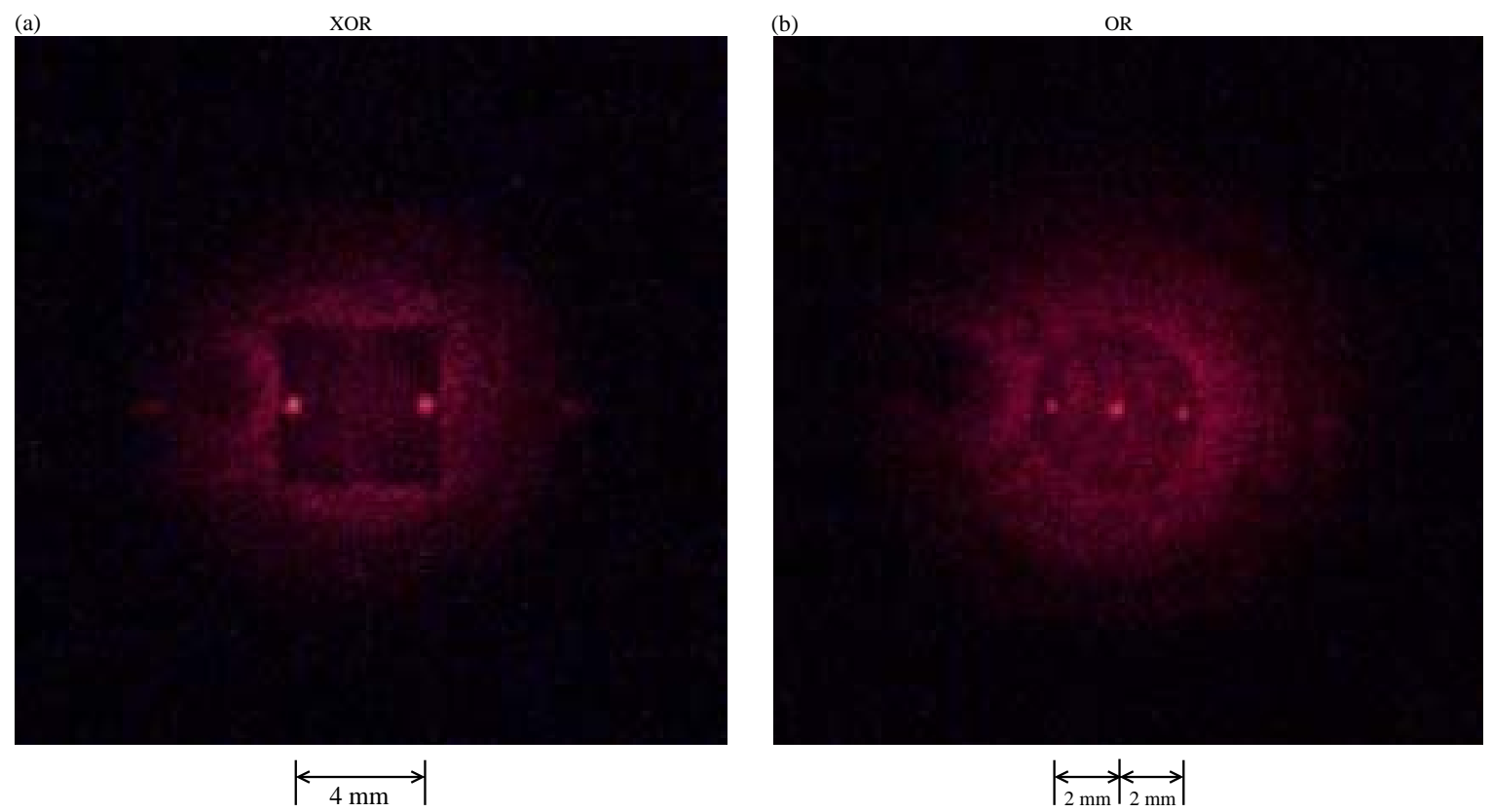

Figure 4.3: A visible light experiment is performed in order to directly verify the intensity distribution at the back focal plane of the XOR pattern. For comparison, an OR pattern obtained by taking the bit-wise OR of a grating and a zoneplate is also fabricated. The effect of this OR pattern is equivalent to that of a grating and a zoneplate placed in tandem, which is the conventional setup for interferometric experiments. Part (a) shows that the intensity distribution at the back focal plane of the XOR pattern consists of only two symmetric off-axis foci, as predicted by the theory. As a comparison, the focal plane intensity distribution of the OR pattern is shown in (b), which has three foci, with one strongest on-axis focus and two weaker off-axis symmetric foci. The grating used by the XOR and OR patterns in this visible experiment has a period of $5 \mu \mathrm{m}$ and the diameter and the outermost zone-width of the zoneplate is $D=5 \mathrm{~mm}$ and $2 \mu \mathrm{m}$, respectively. A He-Ne laser $(\lambda=633 \mathrm{~nm})$ is used for illuminating the XOR and OR patterns. 


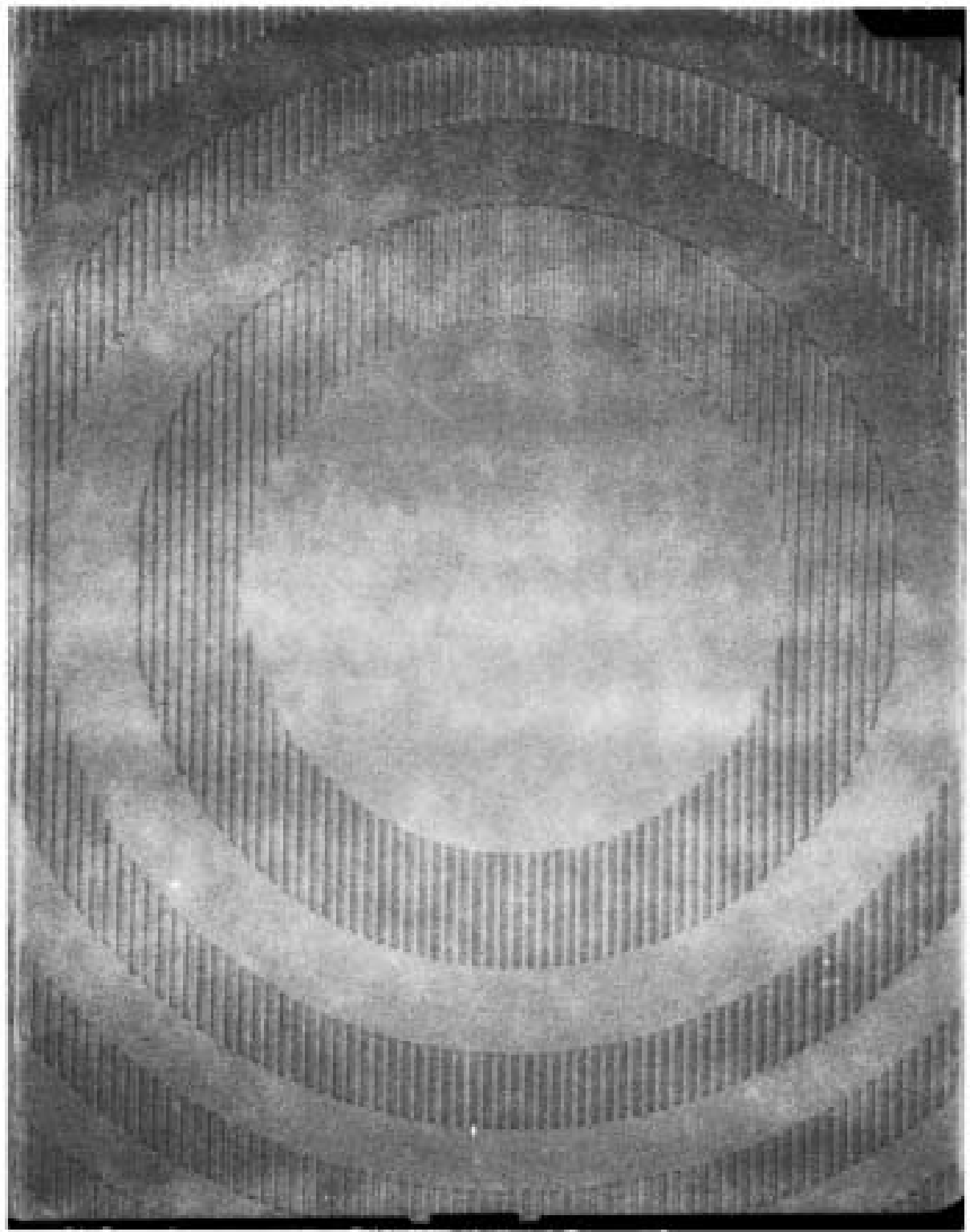

Figure 4.4: A microscope image of the OR pattern used in the experiment with visible light. The grating period is $5 \mu \mathrm{m}$. The zoneplate diameter is $5 \mathrm{~mm}$ and the outermost zone-width is $2 \mu \mathrm{m}$. 


\subsection{First use in EUV interferometry}

The XOR pattern employed in our first application to EUV interferometry is fabricated using the same e-beam lithography tool and an SEM image of the actual pattern is shown in Fig. 4.5. The period $d$ of the grating used here is $16 \mu \mathrm{m}(8 \mu \mathrm{m}$ pitch) and the zoneplate has a diameter $D=400 \mu \mathrm{m}$ and a outermost zone-width $\Delta r=0.2 \mu m$. Undulator beamline 12 at the Advanced Light Source provides the EUV radiation for this measurement. [35] The wavelength at which this measurement was performed is $\lambda=16.53 \mathrm{~nm}(75 \mathrm{eV})$ and the monochromator at the beamline is set at $\lambda / \Delta \lambda=1100$.

This interferometer utilizes the strongest non-zeroth order, i.e. $(m, n)=( \pm 1,1)$, which has a theoretical efficiency of $4 / \pi^{2} \times 1 / \pi^{2}=4 / \pi^{4} \sim 4.1 \%$ as given by Eq. 4.7. Experimentally, the efficiency of this XOR pattern is measured by recording the total counts on the CCD while scanning a knife-like beam-stop transversely across the back focal plane. Starting with the beam-stop placed at the back focal plane such that the entire beam is blocked, as the beam-stop slowly moves aside, the total counts on the CCD increases, allowing fractions of light to pass. The result of this efficiency measurement is shown in Fig. 4.7. The two abrupt steps at the center is caused by the two symmetric off-axis first order foci, $(m, n)=( \pm 1,1)$, being released one at a time by the scanning beam-stop. However, when determining the efficiency of the $(m, n)=( \pm 1,1)$ order, the effect of straight through light needs to be removed. Since the position of the transversely scanning beam-stop is directly proportional to the fraction of the straight through light that passes it, the effect of straight through light can be determined by the constant slope of the two straight sections. After removing the effect of the straight through light by least-square fitting the slope of the two straight sections, the individual strength of the $(m, n)=( \pm 1,1)$ order is shown to be around $4.0 \%$, which agrees with the theoretical value. Note that the definition of diffraction efficiency for this element is the sum of the flux in the two desired orders divided by the total incident flux on the pattern. We measured the diffracted flux to the two desired orders and the total flux through the XOR pattern. The latter is assumed to be half of the total flux incident on the XOR pattern, as 


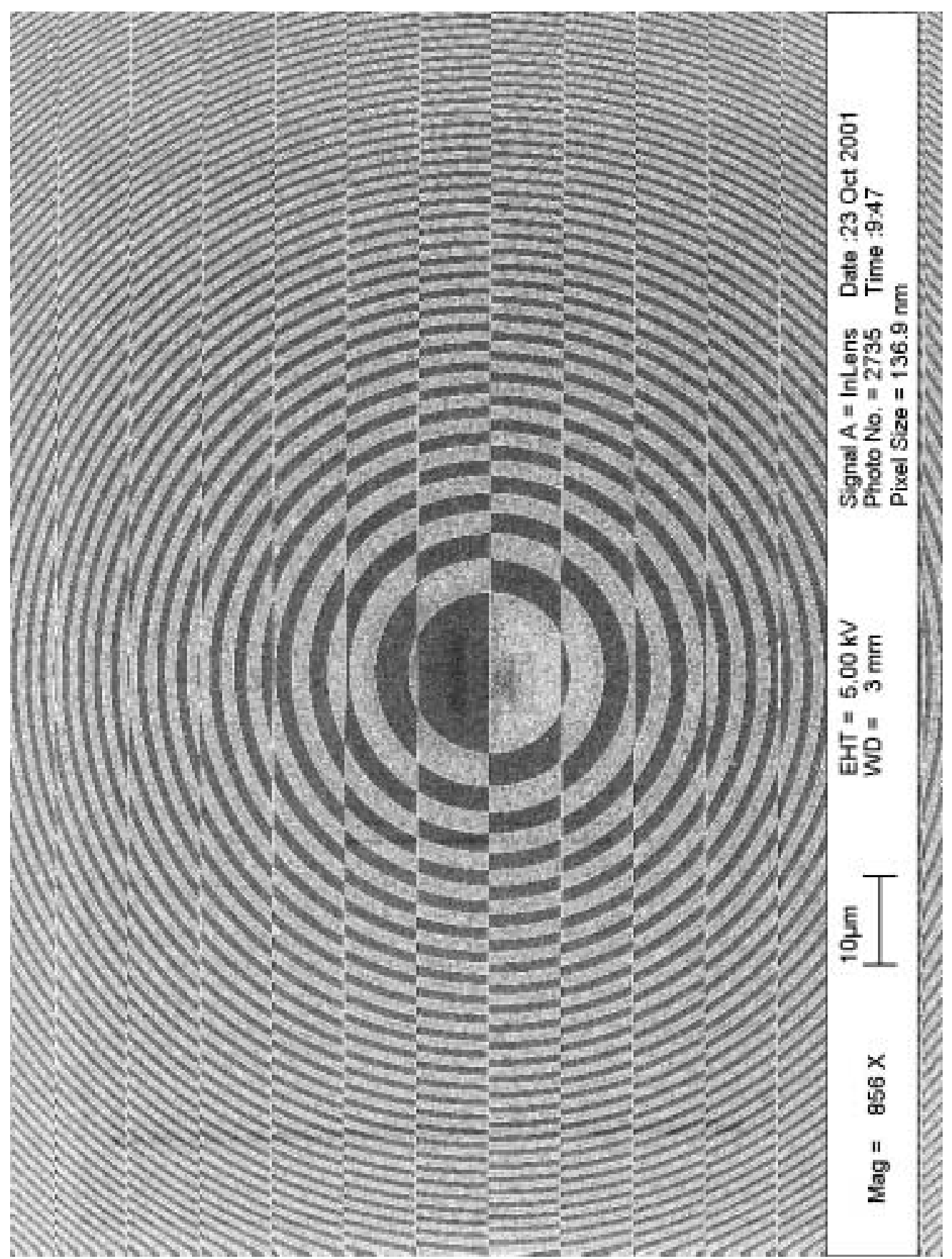

Figure 4.5: The center part of the XOR pattern is shown. This diffractive optical element is obtained by taking the bit-wise XOR of a binary amplitude grating and a binary amplitude zoneplate. The functionality of this XOR pattern is equivalent to that of a binary phase grating overlapping a binary amplitude zoneplate, as discussed in the text. The grating used here has a $16 \mu \mathrm{m}$ period ( $8 \mu \mathrm{m}$ line-space) and the zoneplate has a $400 \mu \mathrm{m}$ diameter and a $0.2 \mu \mathrm{m}$ outermost zone-width. 


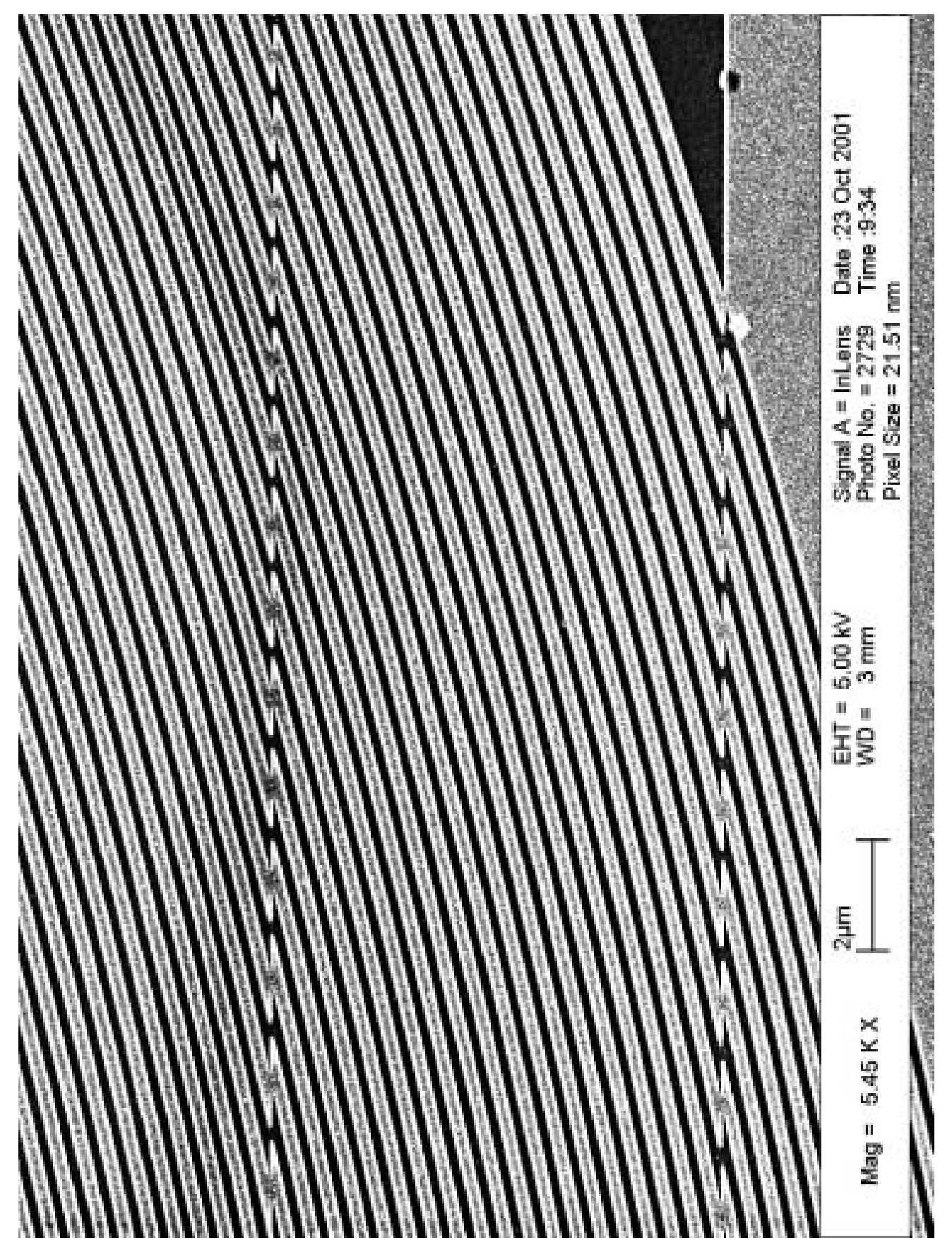

Figure 4.6: The edge of the XOR pattern is shown here. The outmost zone width is seen to be $0.2 \mu \mathrm{m}$ and the alternation of opaque and transparent zones over the grating half-period is also shown. 
half the pattern is transparent. Therefore, the diffraction efficiency is obtained by dividing the diffracted flux in the two orders by twice the total flux through the XOR pattern.

Comparing with the separate binary grating and zoneplate setup, in which the \pm 1 st orders of the grating are being focused by the first order of the zone-plate with a overall efficiency of $1 / \pi^{4} \sim 1.0 \%$, this XOR pattern provides a 4 times improvement in theory. In practice, the required exposure time actually reduces about 10 times due to the fact that the substrates on which these optical elements are fabricated have finite absorption and only one substrate is needed in this case. As will be described in Chapter 5, this improvement in efficiency enables the first direct measurement of refractive index at EUV wavelengths, where the two symmetric first order foci are used as two arms of an interferometer and a direct phase measurement for the dispersive part of the index of refraction is performed [10]. 


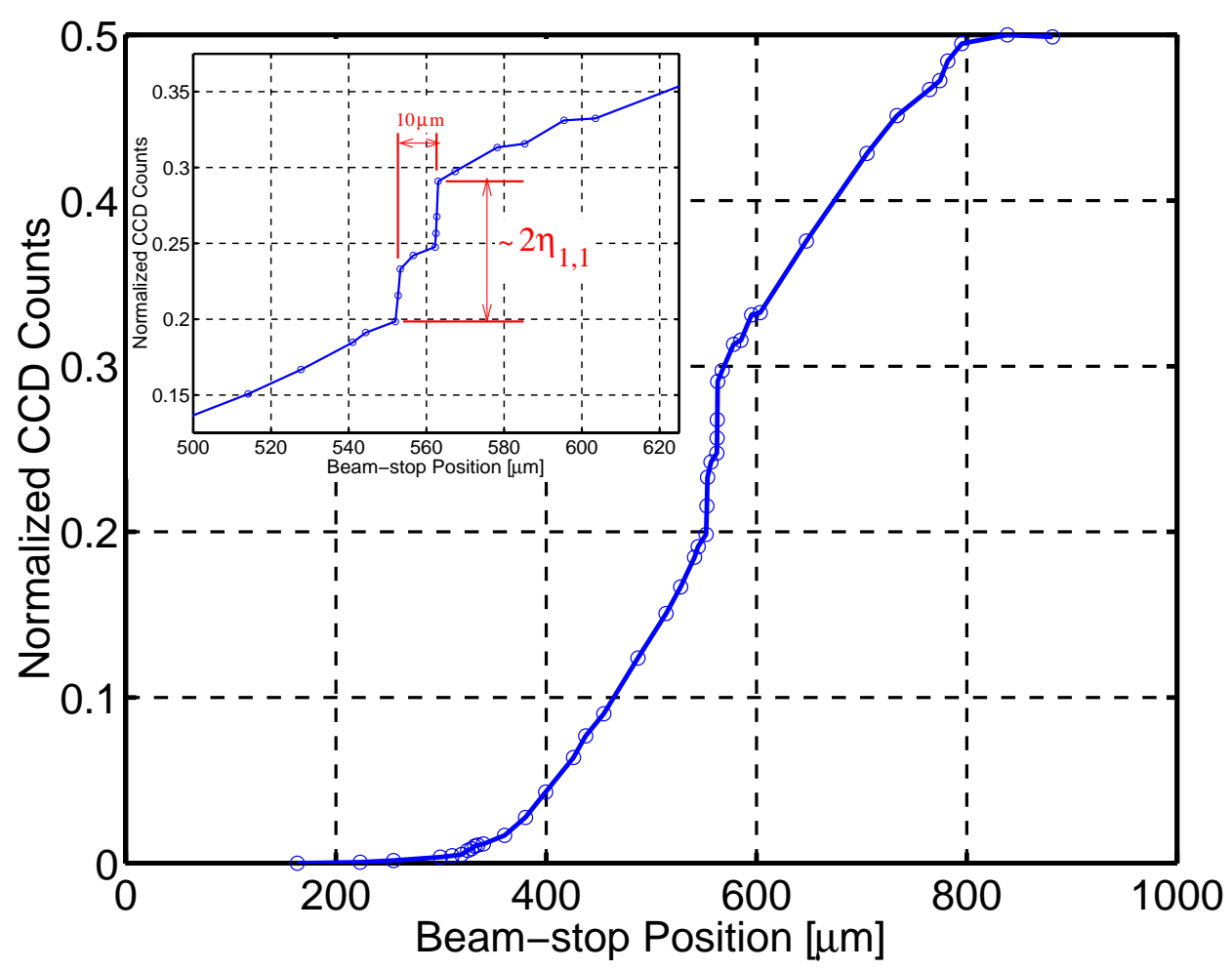

Figure 4.7: The efficiency of this XOR pattern is measured by scanning a knife-like beam-stop across the focal plane. Starting with the beam-stop placed at the back focal plane such that the entire beam is blocked, as the beam-stop slowly moves aside, the total counts on the CCD increases, allowing fractions of light to pass. The constant slope of the two straight sections results from the effect of zeroth order (straight through) light. The two abrupt steps at the center is caused by the two symmetric off-axis first order foci being released one at a time by the beam-stop. Their strength is shown to be around $4.0 \%$, which agrees with the theoretical value.

\subsection{Comparison to the computer generated holo- gram CGH}

A computer generated hologram (CGH) having similar functions can also be constructed. The relationship between CGH and XOR is clarified by showing that even though they are both applications of Fourier optics, with similar functionality, they are of fundamentally different concepts. Specifically, CGH uses the traditional analog concept, while the XOR has an interesting digital aspect. 

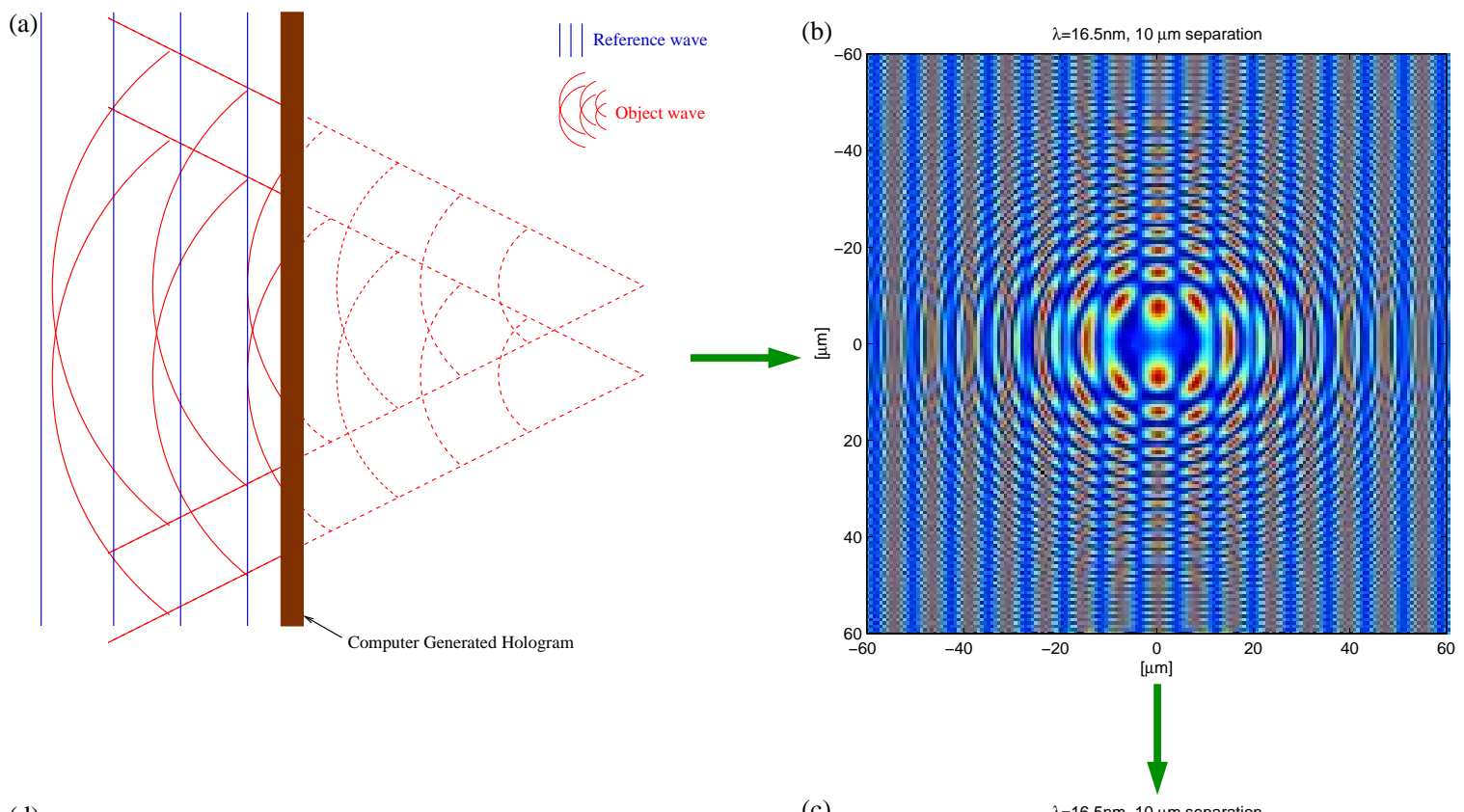

(d)
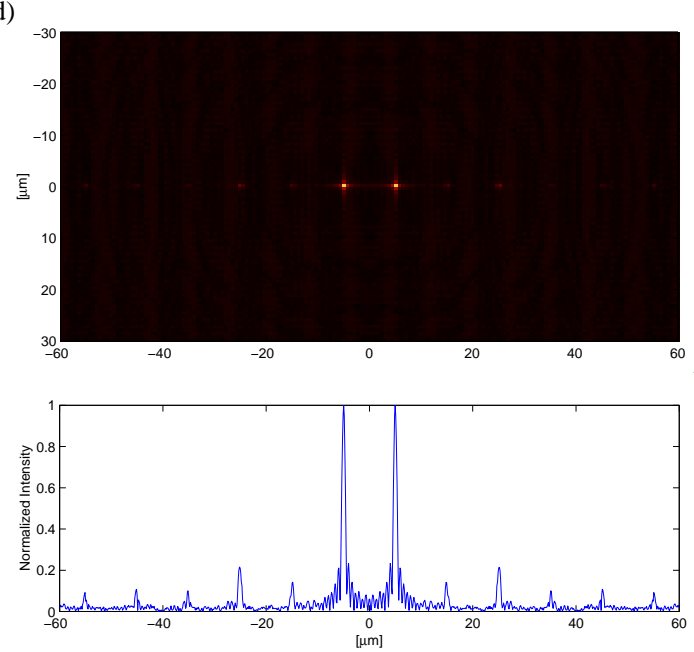

(c)

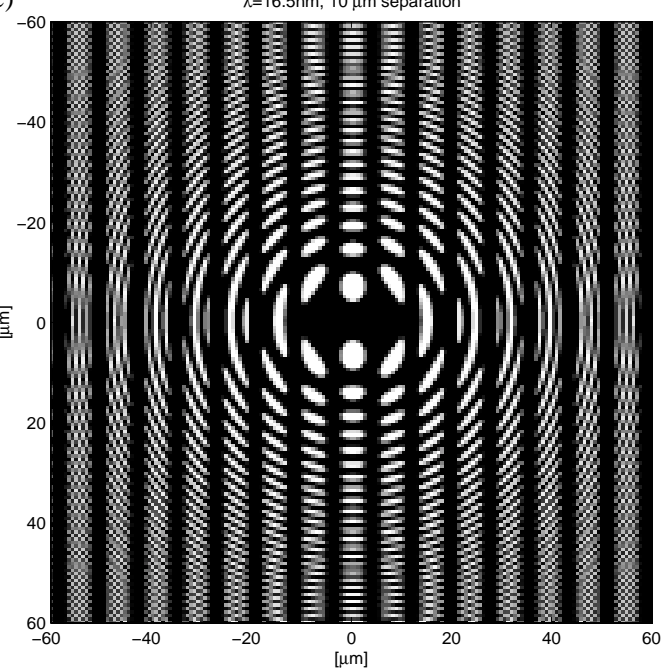

Figure 4.8: In part (a), the object wave (in red) which consists of two converging spherical wavefronts interferes with a reference plane wave (in blue) and the resulting intensity interference pattern, which is usually referred to as Computer Generated Hologram, is shown in (b). This CGH is then binarized for nanofabrication by e-beam lithography. Part (c) shows its binarized form. When illuminated by a uniform plane wave, this optical element reconstructs the object wave (two converging spherical waves) as shown in (d). Note that the two spots are symmetrically off-axis. 
The design concept of CGH is depicted in Fig. 4.8(a). In a computer simulation, the object wave (in red) which consists of two converging spherical wavefronts is encoded by a reference plane wave (in blue) to form an interference pattern (hologram), as shown in Fig. 4.8(b). This CGH, when illuminated by the reference plane wave, will produce two converging spherical wavefronts which can be used for interferometric experiments. Note that these two spherical wavefronts are identical and symmetrically distributed with respect to the optical axis.

To nano-fabricate this CGH, it is necessary to binarize the "smooth" areal interference pattern (Fig. 4.8(b)) into 0's and 1's. This binarized pattern, shown in Fig. 4.8(c), will then be used to produced the CAD file that nano-fabricates the holographic optical element. To see the effect of binarization on the re-constructed wavefront, this binarized holographic optical element is Fresnel-propagated to the plane where the object wave converges to two points and the intensity distribution is shown in Fig. 4.8(d). No significant higher order effects are observed. It is also interesting to compare Fig. 4.2 with Fig. $4.8(\mathrm{c}, \mathrm{d})$ and note that the two different diffractive elements produce similar intensity distributions at the back focal plane.

The CGH can be optimized for optical flux throughput, while the XOR pattern is not specifically designed for maximum efficiency. However, it is very difficult for the CAD program of an electron-beam column to generated a CGH data file due to the large memory requirement imposed by the large amount of very small and irregularly-shaped structures particularly at the outer edge of the CGH. In addition, the finer details required by the $\mathrm{CGH}$ also make it more difficult to nano-fabricate. The XOR pattern provides a more practical solution in that it requires much less computer memory and relatively less stringency in nano-fabrication. For the XOR pattern, the digital data files of the grating and the zoneplate are already accurately calculated and taking the bit-wise XOR operation of the two data files is trivial in computers. 


\subsection{Conclusion}

For the first time a novel diffractive optical element based on Fourier optics techniques has been demonstrated. It is shown, both in theory and in experiment, that by combining two diffractive elements, a grating and a zoneplate, through a bit-wise XOR operation, the resultant optical element produced a new functionality, two symmetric off-axis foci with a higher efficiency. The two symmetric off-axis foci at the back focal plane are used in an EUV experiment to directly measure both the real and imaginary parts of the refractive index. Specifically, it is shown that interferometric experiments that require better contrast and higher coherent power benefit from this XOR design, due to the symmetricalness of the intensity distribution at the back focal plane and the improved overall efficiency, respectively. Although useful at all wavelengths, this pattern has particular value at the short wavelengths of interest here. This group of optical elements shown in this paper brings sophisticated Fourier optical techniques to open new experimental frontiers in an area rich with opportunities on nanometer scales and with element-specific identifications and applications. 


\section{Chapter 5}

\section{Direct index of refraction}

\section{measurements at EUV wavelengths with a novel interferometer}

The first direct measurement of the dispersive part of the refractive index is performed at EUV wavelengths, where absorption is higher as compared with hard x-ray and visible wavelengths. A novel diffractive optical element, the XOR pattern, which combines the functions of a grating and a zoneplate, is used for the interferometer. Both the real and imaginary parts of the complex refractive indices are measured directly by this technique without recourse to Kramers-Kronig transformations. Data for $\mathrm{Al}$ and $\mathrm{Ni}$, in the vicinity of their $\mathrm{L}$ and M-edges, respectively, are presented as first examples of this technique.

\subsection{Introduction}

Refractive indices, $n(\omega)=1-\delta(\omega)+i \beta(\omega)$, in the extreme ultraviolet (EUV) wavelength region are complex, highly absorptive and have strong wavelength (photon energy) dependence [8]. The absorptive part $\beta(\omega)$ of the refractive index at EUV wavelengths is well-tabulated by photo-absorption measurements. However, the real (dispersive) part of the refractive index $\delta(\omega)$ at EUV wavelengths is less accurately known. Interferometry, which can provide independent measurements of $\delta$ and $\beta$, is 
difficult in the EUV/Soft X-Ray(SXR) regions due to high absorption by the many atomic resonances and the lack of high optical quality beam-splitters. Joyeux . $a$. [50] have had some success using an interferometer based on a bi-mirror reflective splitter, but were limited by the trade-off between throughput and spectral resolution. Bonse and Hart [51] have been successful at significantly higher photon energies, where $\beta / \delta \ll 1$, using crystal diffraction techniques. Presently in the EUV/SXR region, knowledge of $\delta$ is determined either indirectly from a Kramers-Kronig transformation [36] of the imaginary (absorptive) part, $\beta(\omega)$ [52-58], or by least-square fitting Fresnel coefficients obtained from reflectance measurements [56,59-62]. Neither of these methods provide an independent measurement of $\delta(\omega)$. The $\delta(\omega)$ values obtained from the Kramers-Kronig relationship depend entirely on the $\beta(\omega)$ values. Because the Kramers-Kronig relationship requires a wide range of spectrum of $\beta(\omega)$ for one to obtain each point of $\delta(\omega)$ on the spectrum, errors in $\beta(\omega)$, especially near absorption edges, affect the accuracy of the resultant $\delta(\omega)$ values. The accuracy of $\delta(\omega)$ values determined from reflectance experiments are sensitive to surface roughness, chemistry and contamination, and to the fact that the accuracy of this fitting depends strongly on $\beta / \delta$, working well only for energies with $\beta / \delta \ll 1$ [56].

Here, an amplitude-division transmission interferometer, which can be used to measure both the dispersive and the absorptive parts of the refractive index independently by determining the phase-shift and the visibility of interferograms, is presented with optimization provided by the XOR pattern discussed in Chapter 4 . Because the determination of $\delta$ directly from the phase-shift is independent of $\beta$, this interferometer can measure $\delta$ across the absorption edges without being affected by sharp spectral variations of the $\beta$ value. In addition, the sample is probed in transmission at normal incidence in this interferometer, thus, it is less sensitive to errors associated with surface roughness, chemistry and contamination, as compared with reflectance measurements, assuming that the thickness of the sample is much greater than that of the surface layer. 


\section{$5.2 \quad$ Interferometry}

\subsubsection{Principle}

Interferometry can be used to extract phase information from an intensity detector. The amplitude-division transmission interferometer employed here utilizes the Fraunhofer diffraction, which states that the field distribution in the far-field can be obtained from a Fourier transform of the aperture field distribution. The underlying principle of operation of this interferometer is discussed here.

Let $\mathbf{U}_{o}(x, y)$ denote the field distribution in the aperture, the resultant far-field field distribution $\mathrm{U}_{1}(u, v)$ is given by [2]

$$
\mathbf{U}_{1}(u, v)=\frac{e^{j k z} e^{j \frac{k}{2 z}\left(u^{2}+v^{2}\right)}}{j \lambda z} \int_{-\infty}^{+\infty} \int_{o}(x, y) e^{-j \frac{2 \pi}{\lambda z}(x u+y v)} d x d y
$$

As shown in Appendix C, the inverse Fourier transform of the far-field intensity distribution, $\left|\mathbf{U}_{1}(u, v)\right|^{2}$, is equal to the auto-correlation of $\mathbf{U}_{o}(x, y)$, i.e.,

$$
\int_{-\infty}^{+\infty}\left|\mathbf{U}_{1}(u, v)\right|^{2} e^{j \frac{2 \pi}{\lambda z}(x u+y v)} d u d v=\int_{-\infty}^{+\infty} \int_{o} \mathbf{U}_{o}\left(x^{\prime}, y^{\prime}\right) \mathbf{U}_{o}^{*}\left(x^{\prime}-x, y^{\prime}-y\right) d x^{\prime} d y^{\prime}
$$

For an intensity detector placed at a distance $z$ away from the aperture, the detected intensity distribution $\left|\mathbf{U}_{1}\right|^{2}$ can be recorded and then inverse Fourier transformed to obtain the auto-correlation of the distribution in the aperture. If the field distribution in the aperture is arranged in such a way that it can be written as

$$
\mathbf{U}_{o}(x, y)=\delta(x, y)+\mathbf{S}(x, y)
$$

then its auto-correlation consists of four parts. The quantities, $\mathbf{S}(x, y)$ and $\mathbf{S}^{*}(-x,-y)$, are present in the auto-correlation of $\mathbf{U}_{o}$, together with the auto-correlation of $\delta(x, y)$ and that of $\mathbf{S}(x, y)$. For the interferometer used here, $\mathbf{S}(x, y)$ is of limited spatial extent and is spatially separated in the inverse Fourier transform domain from the other three components. Therefore, the field distribution $\mathbf{S}(x, y)$ can be extracted and both the amplitude and phase distribution of $\mathbf{S}(x, y)$ can be obtained. 

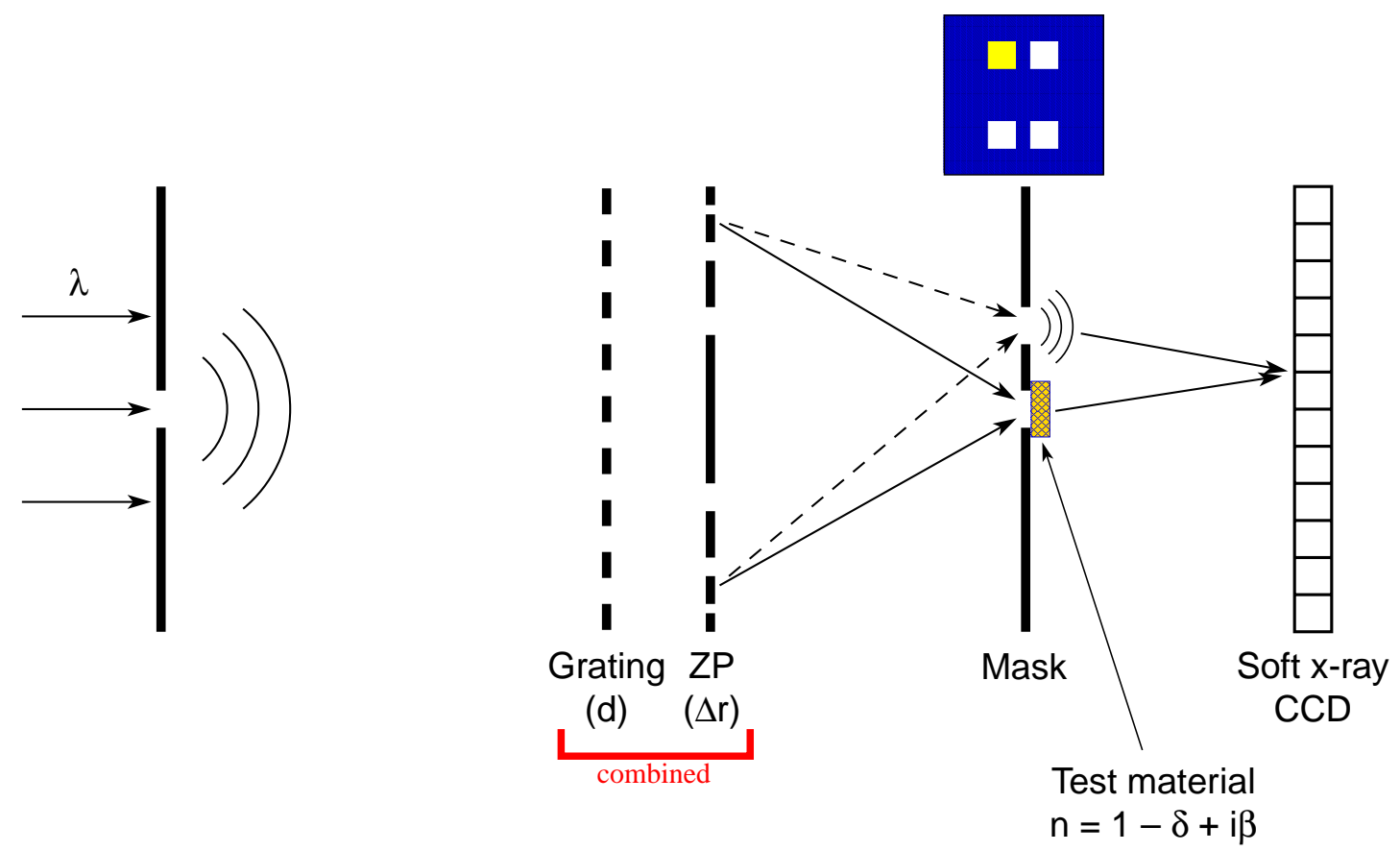

Figure 5.1: Experimental Setup: A $5 \mu \mathrm{m}$ pinhole is place at the focus of the beamlines optics to provide spatially coherent illumination for this interferometer. The grating serves as the beam splitter which effectively creates orders of virtual sources out of the pinhole. The zoneplate then images the pinhole, together with all the virtual sources created by the grating, to the plane of the sample mask. This mask consists of two $5 \mu m \times 5 \mu m$ windows allowing only the two symmetric orders $(m, n)=( \pm 1,1)$ to pass. The test material is then shuffled in and out over one of the windows and an back-thinned EUV-sensitive CCD camera records the respective interferograms for comparison. The functions of the grating and zoneplate have been combined into a single diffractive element following the use of Fourier optical techniques and nanofabrication. 


\subsubsection{The interferometer}

The interferometer used for the measurements presented here is shown conceptually in Fig. 5.1. The size of the pinhole, $5 \mu \mathrm{m}$, is chosen to be smaller than the coherence area of the beam [9], guaranteeing spatially coherent illumination of the interferometer. In concept, the pinhole-diffracted beam then propagates through a binary transmission grating, which serves as a beam-splitter, followed by a zoneplate lens used to focus the beams to the sample plane. As actually used, the grating and zoneplate are combined for improved efficiency into a single diffractive element. This combined optical element provides two side-by-side focal spots of equal intensity, thus ideal for use in interferometric experiments. The properties of the XOR pattern related to the operation of the interferometer are summarized in Sec.5.2.3. A comprehensive study of this XOR pattern is presented in Chapter 4.

The sample mask, consisting of side-by-side window pairs, is placed at the back focal plane of the zoneplate. The window pairs consist of two $5 \mu \mathrm{m} \times 5 \mu \mathrm{m}$ crosssectioned openings. Reference window pairs are free of test material, while other pairs have one window coated with test material. In taking data, one first aligns a reference window pair to the two side-by-side first order focal beam spots and records a reference interferogram. One then moves the sample mask to illuminate a window pair in which one side contains test material, and records the test interferogram. By introducing the test material into one arm of the interferometer, the fringes of the interferogram shift due to the refractive properties of the material, essentially a path integration of $\delta(\omega)$. Additionally, the visibility of the interferogram is reduced due to absorption. The interferograms are recorded on a back-thinned EUV-sensitive CCD camera. The complex index of refraction is determined by comparing these two interferograms for fringe-shift and visibility change, which are directly related to $\delta$ and $\beta$, respectively.

\subsubsection{Novel diffractive optical element}

The XOR pattern described in Chapter 4 is employed here by the interferometer for improved efficiency, which is essential at these highly absorptive wavelengths. The 
use of two sequential diffractive elements (the grating and zoneplate), each of which has a theoretical efficiency to first order of $1 / \pi^{2}$, limits the overall efficiency of the interferometer. This efficiency can be significantly improved by consolidating the functionality of the grating and the zoneplate into a single diffractive optical element. This is done by combining the binary grating and zoneplate through a bit-wise XOR operation. Specifically, the XOR pattern is obtained by first pixelizing the binary grating and zoneplate. Each pixel is either 1 or 0 for transmission and absorption, respectively. The two pixelized patterns are then overlapped and compared pixel by pixel to produce the resulting "XOR" pattern, i.e. at each pixel position, if the pixel values of the grating and zoneplate are the same (both 0's or both 1's), the value of the corresponding pixel on the "XOR" pattern is 0 . Otherwise, the value of the corresponding pixel on the "XOR" pattern is 1 .

The two optical elements used in this XOR pattern, a $50 \%$ duty-cycle binary amplitude grating of period $d$, and a $50 \%$ duty-cycle binary amplitude zoneplate of diameter $D$ and outermost zone-width $\Delta r$, can be represented by

$$
G(x)=\frac{1}{2}\left[1+\operatorname{sgn}\left(\cos \frac{2 \pi x}{d}\right)\right]
$$

and

$$
Z P(x, y)=\frac{1}{2}\left[1+\operatorname{sgn}\left(\cos \frac{\pi\left(x^{2}+y^{2}\right)}{\Delta r(D-\Delta r)}\right)\right]
$$

respectively [2]. Expanding these two patterns in their respective Fourier series and noting that the XOR pattern of the grating and zoneplate can be expressed as $X O R(x, y)=G(x, y)+Z P(x, y)-2 G(x, y) Z P(x, y)$, we have

$$
X O R(x, y)=\frac{1}{2}-2\left[\sum_{\substack{m=-\infty \\ m \neq 0}}^{\infty} \frac{\sin (m \pi / 2)}{m \pi} e^{-i \frac{2 m \pi x}{d}}\right]\left[\sum_{\substack{n=-\infty \\ n \neq 0}}^{\infty} \frac{\sin (n \pi / 2)}{n \pi} e^{-i \frac{n \pi\left(x^{2}+y^{2}\right)}{\Delta r(D-\Delta r)}}\right] .
$$

Examining the first order terms in both the grating and zoneplate, i.e. $(m, n)=$ $( \pm 1,1)$, its efficiency is given by the square of its coefficient $[2(1 / \pi)(1 / \pi)]^{2}=4 / \pi^{4}$, which is a factor of 4 increase in optical throughput as compared with a separate grating and zoneplate setup. Since the membranes on which these optical elements are fabricated have finite absorption, there is an additional gain of efficiency due to the fact that only one membrane is required. 
This combined diffractive element, when illuminated by a uniform wavefront, has the interesting property that it produces two symmetric off-axis focal spots, $(m, n)=$ $( \pm 1,1)$, at the back focal plane of the zoneplate. This can be seen by multiplying the two exponentials in Eq.(5.4) and completing the square for $x$-terms. The separation of these two beam spots is determined by

$$
x_{s} \approx 2 \sin ^{-1}(\lambda / d) \frac{\Delta r D}{\lambda}=\frac{2 \Delta r D}{d} .
$$

Note that this separation is independent of wavelength $\lambda$. Thus as the wavelength is varied for spectral determinations of $\delta$ and $\beta$, the focal length (distance from the XOR pattern to the sample mask) varies, but the lateral separation of the two beam spots remains fixed. Therefore, the index of refraction as a function of wavelength can be obtained simply by translating the same sample mask along the optical axis. 


\subsubsection{Analysis method}

A Fourier transform method $[9,44]$ is used to extract both the phase-shift and visibility from the interferograms. The extracted values are averaged over the full area of the interference pattern.

\section{Phase shift}

The phase shift, $\Delta \phi$, is simply the difference between the two independently reconstructed phase maps from the interferograms. The value of $\delta$ is determined by

$$
\Delta \phi=\frac{2 \pi}{\lambda} t \delta
$$

where $\lambda$ is the wavelength and $t$ is the thickness of the sample.

The Fourier transform method used here to determine the phase shift $\Delta \phi$ has the advantage that the phase information contained in the spatial-frequency modulated fringes is isolated into the first order peaks in the Fourier (i.e. spatial frequency) domain. Filtering of the first order peak in the Fourier domain removes the effect of all stray lights that does not have an identical spatial frequency as that of the fringes. Figure 5.2 shows the analysis process of the Fourier transform method. The interferograms are first Fourier transformed into the Fourier domain, where the first order peaks positioned at the spatial frequency of the fringes are separated from all other spatial frequency components. A Gaussian filter in the Fourier domain is used to extract the first order peak. The extracted first order peak is then inverse Fourier transformed to propagate back to the CCD plane, where the field distribution caused from one of the windows is now obtained. The phase information, wrapped between $\pm \pi$, can now be retrieved from the field distribution. The phase shift between the two interferograms, $\Delta \phi$, is determined simply by subtracting the two wrapped phase distributions. Note that the linear phase terms caused by the off-centered first order peaks are identical in the two wrapped phase distributions, and cancel each other after subtraction. The resultant phase map is only valid over the region where the fringes exist on the CCD and the phase shift number $\Delta \phi$ is quoted as the average over this valid region. 


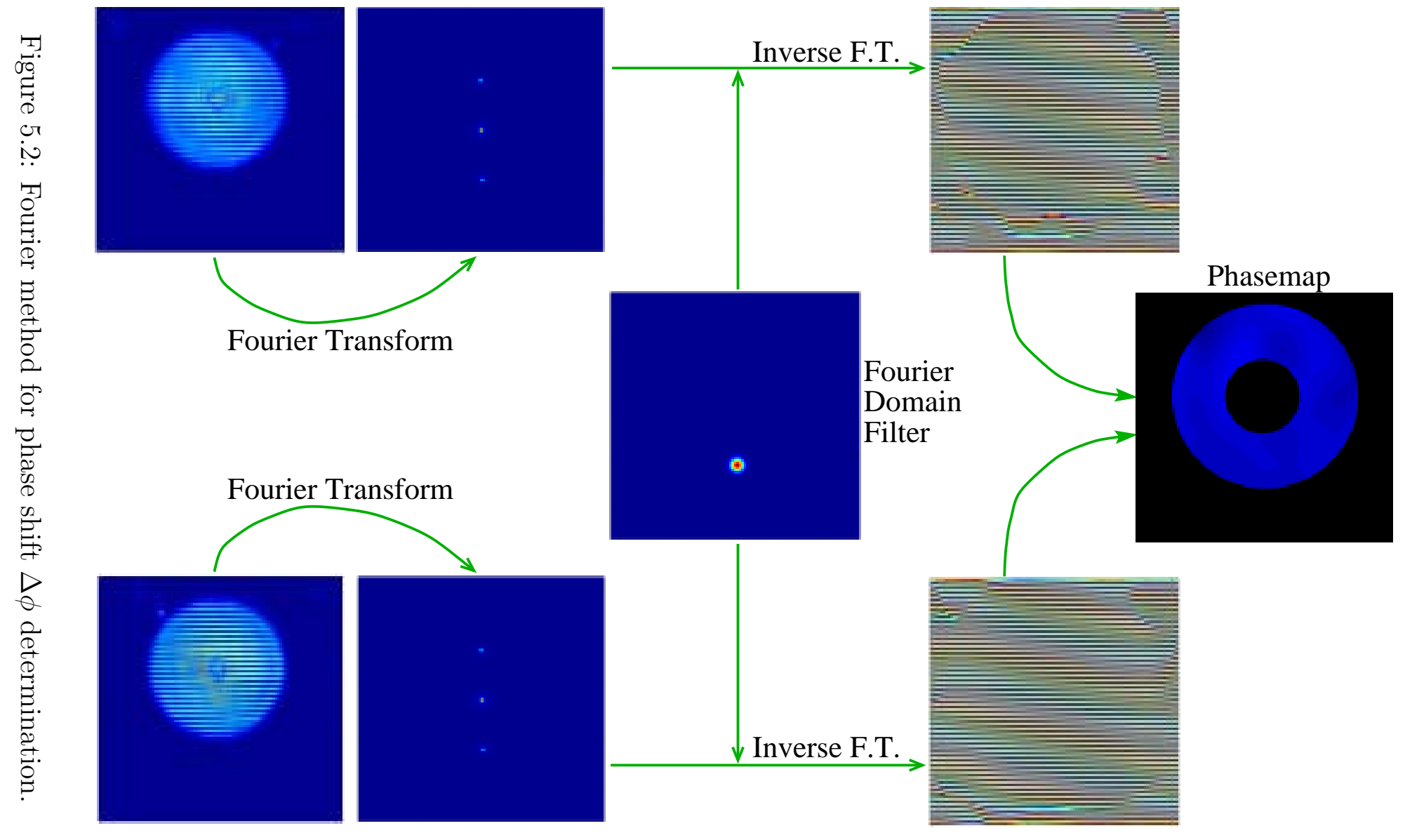




\section{Visibility}

The illumination on the interferometer provided by the pinhole is essentially coherent. The visibility on the CCD is therefore solely determined by the relative intensity of the two focal spots after the window-pair. The relative optical intensity of the two beams, after propagating through the sample mask, is related to observed visibility of the interferogram by

$$
V=\frac{2 \sqrt{\alpha}}{1+\alpha}
$$

where $\alpha$ is the relative intensity after propagating through the sample. From the relative intensity $\alpha$, the value of $\beta$ is obtained by

$$
\alpha=\exp \left\{-\frac{4 \pi}{\lambda} \beta t\right\}
$$

Fourier transform method is again used to extract the visibility from the interferograms. The separation between the two foci is $x_{s}=10 \mu \mathrm{m}$, which is smaller than the pixel size of the CCD $(25 \mu \mathrm{m})$. Therefore, the far-field diffraction patterns from the two foci effectively overlap on the CCD. Additionally, as shown in the analysis of the XOR pattern, the two first order foci are identical. Their far-field diffraction patterns after propagating through the sample mask onto the CCD are again identical apart from their relative intensity. Under these circumstances, the visibility observed on the CCD is directly determined by the relative intensity of the two foci after the sample mask, i.e. $\alpha$. Specifically, the observed intensity on the CCD is give by

$$
I(u, v)=I_{o}(u, v)\left[(1+\alpha)+2 \sqrt{\alpha} \cos \left(\frac{2 \pi}{\lambda z} x_{s} u\right)\right]
$$

where $\lambda$ is the wavelength, $z$ is the distance from the sample mask to the CCD, and $I_{o}(u, v)$ is the diffraction pattern of one of the first order foci had the other focus were blocked. Figure 5.3 shows the coordinate system used in Eq.(5.9). After Fourier transform, terms inside the [·] gives

$$
(1+\alpha) \delta\left(f_{u}, f_{v}\right)+\sqrt{\alpha}\left[\delta\left(f_{u}+\frac{x_{s}}{\lambda z}, f_{v}\right)+\delta\left(f_{u}-\frac{x_{s}}{\lambda z}, f_{v}\right)\right]
$$

The Fourier transform of $I(u, v)$ is given by the convolution of Eq.(5.10) and the Fourier transform of $I_{o}(u, v)$. Therefore, the visibility is obtained in the Fourier 
domain by two times the ratio of the integration under the first-order peak to that under the zeroth-order peak. This analysis method has been used in Chapter 2, where $\alpha=1$ and visibility is solely determined by the magnitude of the complex coherence factor $\left|\boldsymbol{\mu}_{12}\right|$. The visibility obtained by this Fourier method is an average over the entire area where fringes exist.
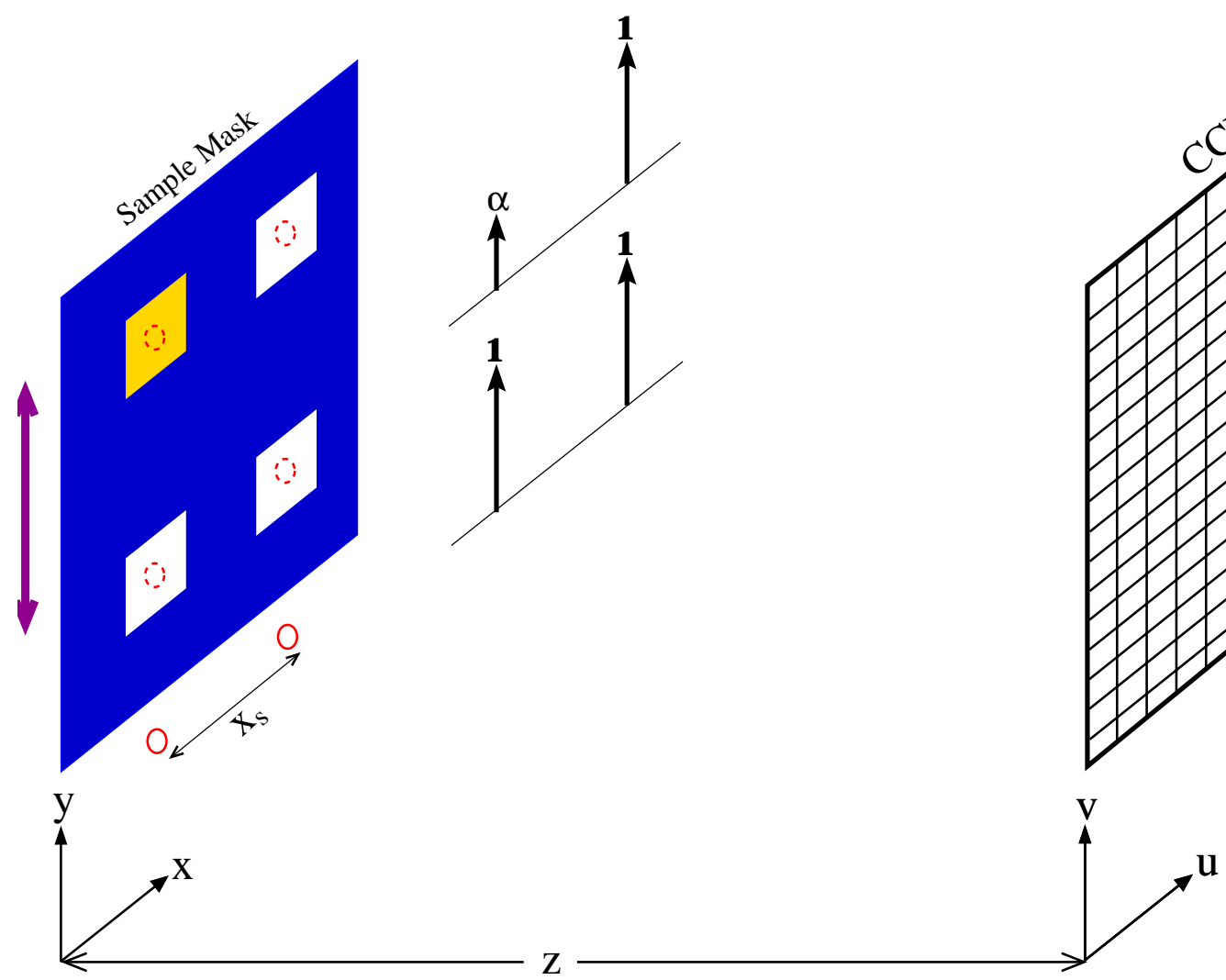

Figure 5.3: Fourier method for determining visibility.

\subsection{Experimental setup}

This experiment is performed at undulator beamline 12.0 of the Advanced Light Source, a third generation synchrotron radiation facility [35]. Undulator radiation provides the required EUV photon flux and, with pinhole spatial filtering, the spatial coherence required for the interferometric experiments. The beamline monochroma- 
tor provides a spectral resolution $\lambda / \Delta \lambda=1100$. As described above, a single XOR diffractive element combining a grating $(d=16 \mu \mathrm{m})$ and zoneplate $(D=400 \mu \mathrm{m}$, $\Delta r=0.2 \mu \mathrm{m})$ is used for optimum efficiency. This new diffractive element is fabricated using electron beam patterning and nanofabrication techniques [22]. The separation of the two beam spots at the back focal plane is $x_{s}=10 \mu \mathrm{m}$.

The sample mask is also fabricated by electron beam lithography on a $100 \mathrm{~nm}$ thick silicon nitride membrane. The thickness of the $S i_{3} N_{4}$ membrane is relatively uniform over the $10 \mu \mathrm{m}$ separation of the two beam spots. The sample is prepared with the test material being evaporated onto the nitride membrane. The thickness of the test material is measured both by a profilometer and a spectral reflectivity system to an accuracy $\pm 1 \mathrm{~nm}$.

The overall distance from the pinhole to the CCD detector is $420 \mathrm{~mm}$ and the distance between the pinhole and the XOR pattern is $110 \mathrm{~mm}$. The separation between the XOR pattern and the sample mask is determined by the first order focal length, which is a function of wavelength $\lambda$. For instance, at $72.5 \mathrm{eV}$, the wavelength is $\lambda=17.1 \mathrm{~nm}$ and the first order focal length is $4.7 \mathrm{~mm}$. Therefore, the distance from the sample mask to the CCD detector is around $300 \mathrm{~mm}$ over the spectral range considered. Given the dimension of the window-pairs, $15 \mu m \times 5 \mu m$, Fraunhofer approximation can be used for the light propagation from the sample mask to the CCD

\subsection{Experimental results}

\subsubsection{Aluminum across its L-edge}

Measured $\delta$ and $\beta$ values for aluminum $67.0 \pm 0.1 \mathrm{~nm}$ thick are shown in Fig. 5.4 in blue. The results obtained with this interferometer resolve the fine aluminum L-edge structure, i.e. the $L_{3}$ edge at $72.7 \mathrm{eV}$ and the $L_{2}$ edge at $73.1 \mathrm{eV}$, in both $\delta$ and $\beta$. Moreover, it is also evident that the shapes of the $\delta$ and $\beta$ curves, though determined independently, are closely related. The sharpest increase in $\beta$ occurs at $72.7 \mathrm{eV}$ which coincides exactly with the dip of the $\delta$ curve at the $L_{3}$ edge. Furthermore, the sharp 
increase of $\beta$ at $73.1 \mathrm{eV}$ also coincides exactly with the dip of the $\delta$ curve at the $L_{2}$ edge. For comparison, the best available data for aluminum refractive indices is shown in red [63], where the $\delta$ value is obtained by Kramers-Kronig transformations of $\beta$ values over a wide spectrum. The two data sets agree fairly well both in $\delta$ and $\beta$.

\subsubsection{Nickel across its M-edge}

Measured $\delta$ and $\beta$ values for nickel of thickness 20.6 $\pm 0.1 \mathrm{~nm}$ are shown in Figs. 5.5. The Nickel $M_{3}$ edge at $66.2 \mathrm{eV}$ is clearly resolved and the $\delta$ and $\beta$ values at this edge are seen to be closely correlated. The error-bars for the nickel data are slightly larger than that of the aluminum data, mainly because of the lower photon flux available in our experiment at the nickel M-edge. Thus, longer exposure times were required resulting in greater noise due to vibration. Improved accuracy at the absorption edge will require increased system stability or increased optical throughput. The best available refractive index values of nickel [64] are shown in red for comparison.

\subsection{Conclusion}

The first direct measurement of the dispersive part of the index of refraction at EUV wavelengths is performed using interferometry. A new diffractive optical element based on Fourier optical techniques is employed in the interferometer for improved efficiency. This interferometer directly measures $\delta$ values at wavelengths where it is desired. No compilation of $\beta$ values over a wide spectral range is needed. As a first example, $\delta$ and $\beta$ values of aluminum and nickel are obtained by this interferometer at wavelengths close to their atomic resonances, i.e. Al L-edge and $\mathrm{Ni}$ M-edge. Extensions of these studies to additional materials ( $\mathrm{Si}, \mathrm{Mo}, \ldots)$, and to shorter wavelengths (1 to $5 \mathrm{~nm}$ ) in the soft x-ray spectral region can be performed with the same interferometer. 


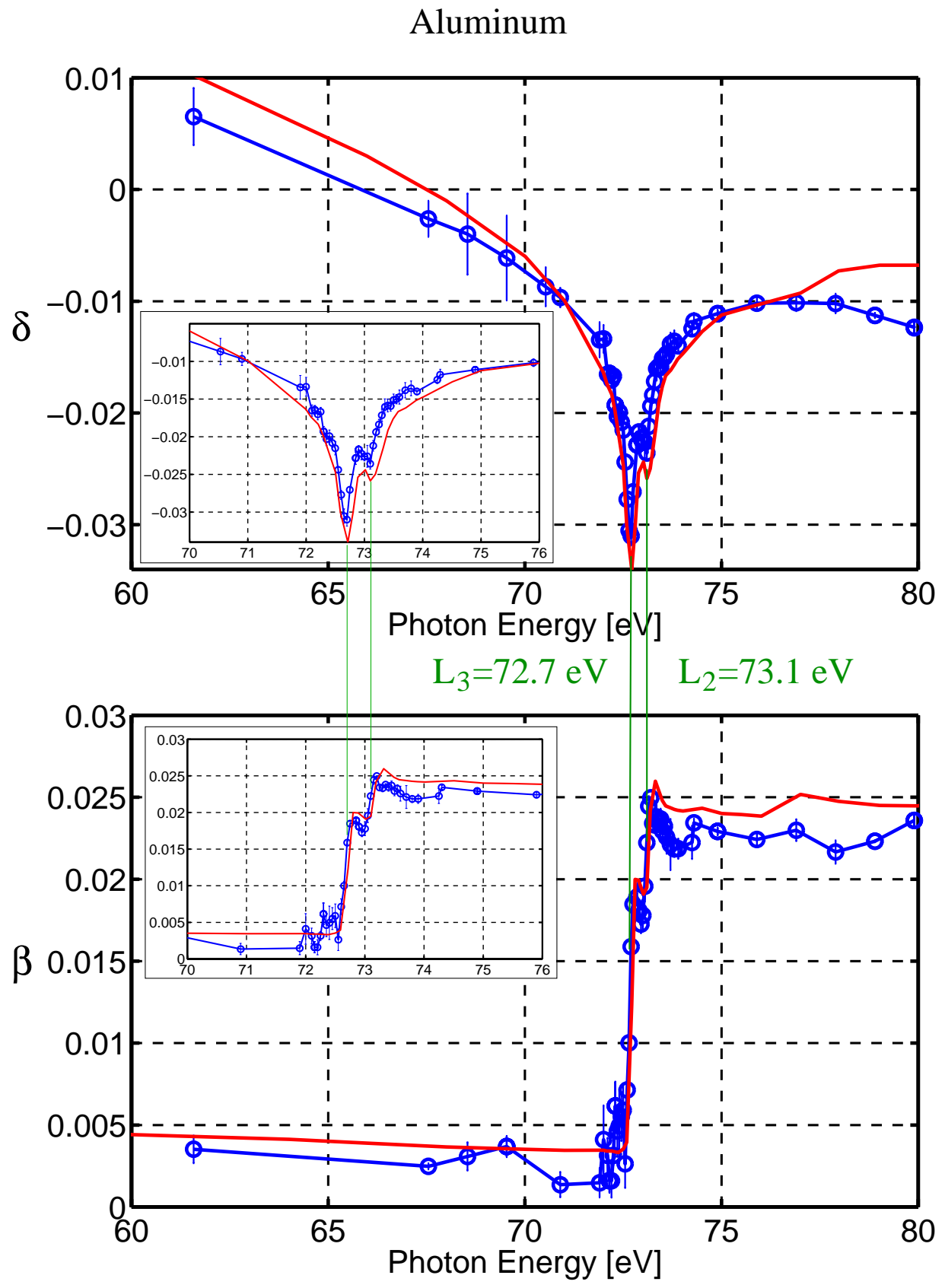

Figure 5.4: The experimental results are shown in blue. It is evident that the aluminum L-edge is resolved both in $\delta$ and $\beta$ where the position of the $L_{2}$ and $L_{3}$ edges are $73.1 \mathrm{eV}$ and $72.7 \mathrm{eV}$, respectively. Note that the values of $\delta$ and $\beta$ are obtained directly (independently) from phase shift and visibility change, respectively. The $\delta$ and $\beta$ values from Ref. [63] (in red) is derived from Kramers-Kronig transformation of a compilation of absorption and reflectance data. The two data sets agree fairly well both in $\delta$ and $\beta$. 


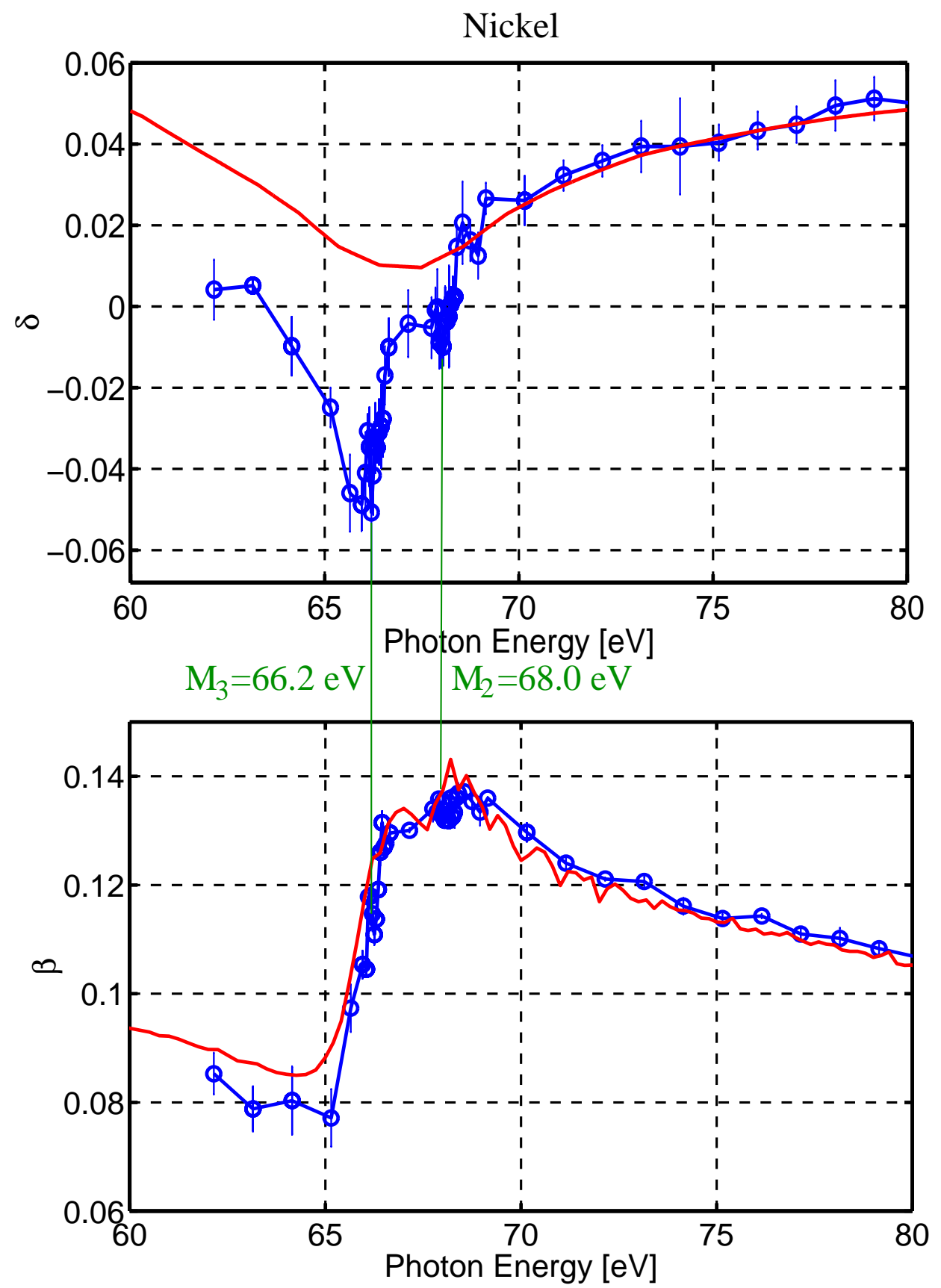

Figure 5.5: Nickel: The experimental results are shown in blue. The $M_{3}$ edge at $66.2 \mathrm{eV}$ is clearly resolved and the $\delta$ and $\beta$ values at this edge are seen to be closely correlated. The typical exposure time $(\sim 200 \mathrm{sec})$ for the nickel interferograms is approximately 10 times longer than that of aluminum due to lower beamline flux at low energy. The stages in the experimental setup drift over longer exposure time, thus causing larger error bars in the nickel data. For comparison, current standard nickel refractive indices are shown in red. 


\section{Chapter 6}

\section{Conclusion}

The higher spatial resolution provided by employing shorter wavelengths corroborates the importance of advancing optical techniques at EUV wavelengths. As a result of this incessant desire of seeing and printing smaller features, new developments in short wavelength optics are continuously emerging. Optical coherence techniques, based on Fourier and statistical optics, constitute a new and pioneering aspect in the progression of EUV optics. In this dissertation, experiments that confirm and characterize the spatial coherence properties of EUV undulator radiation are presented. In addition, the first Fourier optical element, the XOR pattern, is described and utilized in the first direct measurement of refractive indices in this wavelength region.

Understanding the spatial coherence properties of a radiation source is essential to the design and implementation of concomitant optical systems. Unlike visible light optics, the spatial coherence properties of the relatively new EUV radiation sources are not well understood. Therefore, the first experiment in this dissertation was aimed at characterizing the spatial coherence properties of EUV undulator radiation using the Thompson-Wolf two-pinhole method. The result of these experiments demonstrate that, with appropriate spatial filtering, high spatial coherence at EUV wavelengths region is achievable with undulator radiation at third generation (small electron beam phase space) synchrotron facilities. New opportunities are thus presented for experiments that require high spatial coherence, e.g. high resolution interferometry $[41,42,65]$, holography [66, 67], coherent scattering [68, 69], and fo- 
cused microbeam analysis [70], etc. In addition, this experimental characterization uncovers an interesting characteristic of the undulator radiation, i.e. the difference in the measured horizontal and vertical coherence profile. These studies have verified that the Zernike approximation, which is most standard in visible light optics, is not applicable here. This is a consequence of the exceedingly small vertical source size of the undulator. The effect of this small vertical source size on the resultant numerical coherence distribution at the image plane of the condenser evokes further theoretical studies that are described in Chapter 3. For the EUV undulator that acts as a condenser which re-images the incoherent source to its conjugate plane, rigorous spatial coherence propagation based on the Huygens-Fresnel principle is necessary and used in Chapter 3 in order to obtain the correct mutual intensity distribution.

As statistical optics is utilized in Chapter 2 and 3, Chapter 4 and 5 exploit Fourier optical techniques. A novel diffractive optical element based on Fourier optics, the XOR pattern, is demonstrated for the first time. This XOR pattern substantially improves the flux throughput and therefore resolves the high absorption problems at these wavelengths. Another interesting property of this XOR pattern is its generation of two symmetrical, off-axis, foci. This symmetricalness, together with the improved optical throughput, makes the XOR pattern an ideal candidate for interferometry at EUV wavelengths. Previously, the high absorption and the lack of high-quality beamsplitters in the EUV wavelength region precluded attempts at direct at-wavelength interferometric measurements of refractive indices. With this new XOR pattern, the first direct measurement at EUV wavelengths of the dispersive part $(\delta)$ of the refractive index is performed for aluminum around its $L$ edge, and nickel around its $M$ edge. The measured values are in good agreement, but more detailed and more accurate than current standards, which are obtained indirectly from Kramers-Kronig transformations of the absorption data. The use of this new XOR pattern brings sophisticated Fourier optical techniques to shorter wavelengths. Furthermore, the demonstrated capability in the highly absorptive EUV wavelength region opens up new opportunities on nanometer scale applications and element-specific identifications.

This dissertation on coherence techniques has accomplished its goal in advancing two frontiers of short wavelength optics: coherence characterization of radiation 
sources and the introduction of novel optical elements. A thorough understanding of the spatial coherence properties of the undulator radiation has been achieved through both experimental and theoretical studies. The invention of a novel XOR pattern demonstrated the first application of the Fourier optical techniques in short wavelength optics. This invention also enabled the first direct measurement of the dispersive part of refractive indices at EUV wavelengths. Optical coherence techniques introduced in this dissertation have advanced the field of short wavelength optics and will continue to play an essential role in the development of short wavelength optics. 


\section{Appendix A}

\section{Free space coherence propagation of an incoherent source with}

\section{Gaussian-shaped intensity}

\section{distribution}

\section{A.1 Fourier transform of a Gaussian distribution function}

A Gaussian distribution with parameter $\sigma$ is given by

$$
g(t)=\frac{1}{\sigma \sqrt{2 \pi}} \exp \left(-\frac{t^{2}}{2 \sigma^{2}}\right)
$$

Note that this distribution function is normalized, i.e.

$$
\int_{-\infty}^{+\infty} g(t) d t=1
$$

The Fourier transform of this normalized Gaussian function is given by

$$
G(f)=\int_{-\infty}^{+\infty} \frac{1}{\sigma \sqrt{2 \pi}} e^{-t^{2} / 2 \sigma^{2}} e^{-j 2 \pi f t} d t
$$


Further evaluate this integral to get

$$
\begin{aligned}
G(f)= & \frac{1}{\sigma \sqrt{2 \pi}} \int_{-\infty}^{+\infty} \exp \left\{-\frac{1}{2 \sigma^{2}}\left[t^{2}+j 4 \pi \sigma^{2} f t\right]\right\} d t \\
= & \frac{1}{\sigma \sqrt{2 \pi}} \int_{-\infty}^{+\infty} \exp \left\{-\frac{1}{2 \sigma^{2}}\left[\left(t+j 2 \pi \sigma^{2} f\right)^{2}\right]-2 \pi^{2} \sigma^{2} f^{2}\right\} d t \\
= & \frac{\exp \left(-2 \pi^{2} \sigma^{2} f^{2}\right)}{\sigma \sqrt{2 \pi}} \int_{-\infty}^{+\infty} \exp \left\{-\frac{1}{2 \sigma^{2}}\left[\left(t+j 2 \pi \sigma^{2} f\right)^{2}\right]\right\} d\left(t+j 2 \pi \sigma^{2} f\right) \\
= & \exp \left(-2 \pi^{2} \sigma^{2} f^{2}\right) \\
& g(t)=\frac{1}{\sigma \sqrt{2 \pi}} \exp \left(-\frac{t^{2}}{2 \sigma^{2}}\right) \Longleftrightarrow G(f)=\exp \left(-2 \pi^{2} \sigma^{2} f^{2}\right)
\end{aligned}
$$

Notice that because $g(t)$ is an even function, its Fourier tranform $G(f)$ is a real-valued function.

\section{A.2 Coherence distribution after propagating from the undulator exit-plane}

\section{A.2.1 Van Cittert-Zernike Theorem}

The Van Cittert-Zernike theorem governs the coherence propagation of an incoherent source.

$$
\mathbf{J}\left(x_{1}, y_{1} ; x_{2}, y_{2}\right)=\frac{\kappa e^{-j \psi}}{(\lambda z)^{2}} \iint_{-\infty}^{+\infty} I_{s}(\xi, \eta) e^{-j \frac{2 \pi}{\lambda z}(\xi \Delta x+\eta \Delta y)} d \xi d \eta
$$

where

$$
\kappa=\frac{\lambda^{2}}{\pi} \quad \text { and } \quad \psi=\frac{\pi}{\lambda z}\left[\left(x_{2}^{2}+y_{2}^{2}-x_{1}^{2}-y_{1}^{2}\right)\right]
$$

Note that here $(\Delta x, \Delta y)$ is defined as

$$
(\Delta x, \Delta y)=\left(x_{1}-x_{2}, y_{1}-y_{2}\right)
$$

which is different from that in Goodman [3], where $(\Delta x, \Delta y)$ is defined as $\left(x_{2}-x_{1}, y_{2}-\right.$ $y_{1}$ ). Therefore, the sign of the exponential term in Eq.(A.6) changes accordingly. 
Notice that $\mathbf{J}\left(x_{1}, y_{1} ; x_{2}, y_{2}\right)$ is in general a function of the four coordinates $\left(x_{1}, y_{1} ; x_{2}, y_{2}\right)$, but its modulus of $\left|\mathbf{J}\left(x_{1}, y_{1} ; x_{2}, y_{2}\right)\right|$ is a function of the coordinate difference $(\Delta x, \Delta y)$ only, i.e.

$$
\begin{aligned}
J(\Delta x, \Delta y) & \equiv\left|\mathbf{J}\left(x_{1}, y_{1} ; x_{2}, y_{2}\right)\right| \\
& =\frac{\kappa}{(\lambda z)^{2}}\left|\iint_{-\infty}^{+\infty} I_{s}(\xi, \eta) e^{-j \frac{2 \pi}{\lambda z}(\xi \Delta x+\eta \Delta y)} d \xi d \eta\right|
\end{aligned}
$$

The Van Cittert-Zernike theorem can be expressed in the normalized form as follows, using the complex coherence factor $\boldsymbol{\mu}$,

$$
\begin{aligned}
\boldsymbol{\mu}\left(x_{1}, y_{1} ; x_{2}, y_{2}\right) & \equiv \frac{\mathbf{J}\left(x_{1}, y_{1} ; x_{2}, y_{2}\right)}{\mathbf{J}\left(x_{1}, y_{1} ; x_{1}, y_{1}\right)} \\
& =\frac{e^{i \psi} \iint_{\infty}^{+\infty} I_{s}(\xi, \eta) e^{-j \frac{2 \pi}{\lambda z}(\xi \Delta x+\eta \Delta y)} d \xi d \eta}{\int \mathcal{L}_{\infty}^{+\infty} I_{s}(\xi, \eta) d \xi d \eta}
\end{aligned}
$$

Note again that the $\boldsymbol{\mu}\left(x_{1}, y_{1} ; x_{2}, y_{2}\right)$ is in general a function of the four coordinates $\left(x_{1}, y_{1} ; x_{2}, y_{2}\right)$, and its modulus $\left|\boldsymbol{\mu}\left(x_{1}, y_{1} ; x_{2}, y_{2}\right)\right|$ is a function of the coordinate difference $(\Delta x, \Delta y)$ only, i.e.

$$
\begin{aligned}
\mu(\Delta x, \Delta y) & \equiv\left|\boldsymbol{\mu}\left(x_{1}, y_{1} ; x_{2}, y_{2}\right)\right| \\
& =\left|\frac{\iint_{\infty}^{+\infty} I_{s}(\xi, \eta) e^{-j \frac{2 \pi}{\lambda z}(\xi \Delta x+\eta \Delta y)} d \xi d \eta}{\iint_{\infty}^{+\infty} I_{s}(\xi, \eta) d \xi d \eta}\right|
\end{aligned}
$$

\section{A.2.2 Incoherent source with Gaussian intensity distribution}

An incoherent source, whose intensity distribution is Gaussian with $\left(\sigma_{\xi}, \sigma_{\eta}\right)$, can be expressed as

$$
I_{s}(\xi, \eta)=C \exp \left\{-\left(\frac{\xi^{2}}{2 \sigma_{\xi}^{2}}+\frac{\eta^{2}}{2 \sigma_{\eta}^{2}}\right)\right\}
$$

Given this Gaussian intensity source, its coherence distribution after propagating a distance $z$ can be determined by Eq.(A.10). First, the integral in the numerator is 
evaluated to be

$$
\begin{aligned}
& \iint_{-\infty}^{+\infty} C \exp \left\{-\left(\frac{\xi^{2}}{2 \sigma_{\xi}^{2}}+\frac{\eta^{2}}{2 \sigma_{\eta}^{2}}\right)\right\} e^{-j \frac{2 \pi}{\lambda z}(\xi \Delta x+\eta \Delta y)} d \xi d \eta \\
= & C \sigma_{\xi} \sqrt{2 \pi} \exp \left\{-2 \pi^{2} \sigma_{\xi}^{2}\left(\frac{\Delta x}{\lambda z}\right)^{2}\right\} \times \sigma_{\eta} \sqrt{2 \pi} \exp \left\{-2 \pi^{2} \sigma_{\eta}^{2}\left(\frac{\Delta y}{\lambda z}\right)^{2}\right\} \\
= & C 2 \pi \sigma_{\xi} \sigma_{\eta} \exp \left\{-\left[\frac{(\Delta x)^{2}}{2\left(\lambda z / 2 \sigma_{\xi} \pi\right)^{2}}+\frac{(\Delta y)^{2}}{2\left(\lambda z / 2 \sigma_{\eta} \pi\right)^{2}}\right]\right\}
\end{aligned}
$$

Note that Eq.(A.5) is used with $\left(f_{\xi}, f_{\eta}\right)$ being given by

$$
\left(f_{\xi}, f_{\eta}\right)=\left(\frac{\Delta x}{\lambda z}, \frac{\Delta y}{\lambda z}\right)
$$

to arrive at the above result. Also note that the result of this double-integral is real-valued.

Secondly, Eq.(A.2) is used to obtain the denuminator as

$$
\iint_{-\infty}^{+\infty} C \exp \left\{-\left(\frac{\xi^{2}}{2 \sigma_{\xi}^{2}}+\frac{\eta^{2}}{2 \sigma_{\eta}^{2}}\right)\right\} d \xi d \eta=C 2 \pi \sigma_{\xi} \sigma_{\eta}
$$

Therefore, the resultant complex coherence factor $\boldsymbol{\mu}$ after propagating a Gaussianshaped incoherent source by a distance $z$ is

$$
\boldsymbol{\mu}\left(x_{1}, y_{1} ; x_{2}, y_{2}\right)=e^{-j \psi} \exp \left\{-\left[\frac{(\Delta x)^{2}}{2\left(\lambda z / 2 \sigma_{\xi} \pi\right)^{2}}+\frac{(\Delta y)^{2}}{2\left(\lambda z / 2 \sigma_{\eta} \pi\right)^{2}}\right]\right\}
$$

and its modulus $|\boldsymbol{\mu}|$ is given by

$$
\begin{aligned}
\mu(\Delta x, \Delta y) & \equiv\left|\boldsymbol{\mu}\left(x_{1}, y_{1} ; x_{2}, y_{2}\right)\right| \\
& =\exp \left\{-\left[\frac{(\Delta x)^{2}}{2\left(\lambda z / 2 \sigma_{\xi} \pi\right)^{2}}+\frac{(\Delta y)^{2}}{2\left(\lambda z / 2 \sigma_{\eta} \pi\right)^{2}}\right]\right\} .
\end{aligned}
$$

To conclude, given an incoherent source of Gaussian intensity distribution $\left(\sigma_{\xi}, \sigma_{\eta}\right)$, the modulus of the complex coherence factor at a distance $z$ away from the incoherent source is again Gaussian in coordinate difference $(\Delta x, \Delta y)$ with

$$
\left(\sigma_{x}^{C}, \sigma_{y}^{C}\right)=\left(\frac{\lambda z}{2 \pi \sigma_{\xi}}, \frac{\lambda z}{2 \pi \sigma_{\eta}}\right)
$$




\section{Appendix B}

\section{Pupil function of a thin lens}

Under the paraxial approximation, the amplitude transmittance function of a thin lens, $\mathbf{t}_{l}(x, y)$, can be written as,

$$
\mathbf{t}_{l}(x, y)=\mathbf{P}(x, y) \exp \left[-j \frac{\pi}{\lambda f}\left(x^{2}+y^{2}\right)\right]
$$

where the

a $\mathbf{P}(x, y)$, accounts for the finite aperture of the thin lens and the exponential term represents the refractive function of a thin lens of focal length $f$. The phase of $\mathbf{P}$ accommodates lens aberrations, and $|\mathbf{P}|$ may vary within the aperture to account for apodizations. Also note that $\mathbf{P}=0$ outside the lens aperture.

The complex pupil function, $\mathbf{P}(x, y)$, can be written explicitly as

$$
\mathbf{P}(x, y)=|\mathbf{P}(x, y)| \exp \{j k \Phi(x, y)\}
$$

where $k=2 \pi / \lambda$ and $\Phi(x, y)$ is the aberration function. 


\section{Appendix C}

\section{Holographic interferometry -}

\section{Retrieving phase information from an intensity detector}

\section{C.1 Mathematical preliminaries}

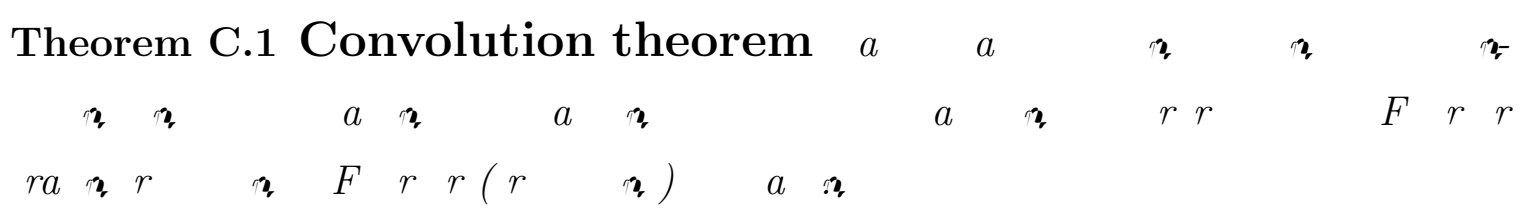

Proof

Given two Fourier transform pairs $g(t) \Leftrightarrow G(f)$ and $h(t) \Leftrightarrow H(f)$, i.e.

$$
G(f)=\int_{-\infty}^{+\infty} g(t) e^{-j 2 \pi f t} d t
$$

and similarly for $h(t) \Leftrightarrow H(f)$, their convolution in time domain is defined by

$$
g \otimes h(t) \equiv \int_{-\infty}^{+\infty} g(\tau) h(t-\tau) d \tau
$$


Fourier transform to get

$$
\begin{aligned}
\mathcal{F}[g \otimes h(t)](f) & =\int_{-\infty}^{+\infty}\left(\int_{-\infty}^{+\infty} g(\tau) h(t-\tau) d \tau\right) e^{-j 2 \pi f t} d t \\
& =\int_{-\infty}^{+\infty} d \tau g(\tau) e^{-j 2 \pi f \tau} \int_{-\infty}^{+\infty} d(t-\tau) h(t-\tau) e^{-j 2 \pi f(t-\tau)} \\
& =G(f) H(f)
\end{aligned}
$$

Theorem C.2 Auto-correlation theorem $a \quad r$ $r$.

\section{Proof}

Let $H(f) \equiv G^{*}(f)$, then

$$
\begin{aligned}
h(t) & =\int_{-\infty}^{+\infty} G^{*}(f) e^{j 2 \pi f t} d f \\
& =\left(\int_{-\infty}^{+\infty} G(f) e^{-j 2 \pi f t} d f\right)^{*} \\
& =(g(-t))^{*}
\end{aligned}
$$

Therefore,

$$
\begin{aligned}
|G(f)|^{2}=G(f) G^{*}(f) \Longleftrightarrow & g(t) \otimes g^{*}(-t) \\
= & \int_{-\infty}^{+\infty} g(\tau) g^{*}(-(t-\tau)) d \tau \\
= & \int_{-\infty}^{+\infty} g(\tau) g^{*}(\tau-t) d \tau
\end{aligned}
$$




\section{C.2 Holographic interferometry}

\section{C.2.1 Far-field coherent field propagation}

In the far-field, i.e. under the Fraunhofer approximation, which requires that

$$
z \gg \frac{k\left(x^{2}+y^{2}\right)_{\max }}{2}
$$

where $k=2 \pi / \lambda$, the field distribution $\mathbf{U}_{1}(u, v)$ at a distance $z$ away from the aperture can be obtained by Fourier transforming the aperture field distribution $\mathbf{U}_{o}(x, y)$, i.e.,

$$
\mathbf{U}_{1}(u, v)=\frac{e^{j k z} e^{j \frac{k}{2 z}\left(u^{2}+v^{2}\right)}}{j \lambda z} \int_{-\infty}^{+\infty} \mathbf{U}_{o}(x, y) e^{-j \frac{2 \pi}{\lambda z}(x u+y v)} d x d y
$$

Note that the coordinate system is defined in Fig. 5.3.

Therefore, if the far-field field distribution is given, the field distribution in the aperture can be obtained. However, a regular detector can only record the distribution, not the field distribution. Using the distribution recorded in the far-field, an inverse Fourier transform gives the auto-correlation of the aperture field distribution. As will be described next, holographic interferometry utilizes this property to retrieve distribution in the aperture from an intensity detector placed in the far-field.

\section{C.2.2 From intensity distribution to auto-correlation}

Given the intensity distribution in the far-field recorded on a detector, i.e. $\left|\mathbf{U}_{1}\right|^{2}$, re-write as follows,

$$
\begin{aligned}
& \left|\mathbf{U}_{1}(u, v)\right|^{2}=\mathbf{U}_{1}(u, v) \mathbf{U}_{1}^{*}(u, v) \\
= & \frac{1}{\lambda^{2} z^{2}}\left[\iint_{-\infty}^{+\infty} \mathbf{U}_{o}\left(x^{\prime}, y^{\prime}\right) e^{-j \frac{2 \pi}{\lambda z}\left(x^{\prime} u+y^{\prime} v\right)} d x^{\prime} d y^{\prime}\right]\left[\iint_{-\infty}^{+\infty} \mathbf{U}_{o}\left(x^{\prime \prime}, y^{\prime \prime}\right) e^{-j \frac{2 \pi}{\lambda z}\left(x^{\prime \prime} u+y^{\prime \prime} v\right)} d x^{\prime \prime} d y^{\prime \prime}\right]^{*}
\end{aligned}
$$


Perform the inverse Fourier transform of $\left|\mathbf{U}_{1}(u, v)\right|^{2}$, denoted as $\mathbf{A}_{U_{o}}(x, y)$,

$$
\begin{aligned}
\mathbf{A}_{U_{o}}(x, y) \equiv & \iint_{-\infty}^{+\infty}\left|\mathbf{U}_{1}(u, v)\right|^{2} e^{j \frac{2 \pi}{\lambda z}(x u+y v)} d u d v \\
= & \frac{1}{\lambda^{2} z^{2}} \iint_{-\infty}^{+\infty}\left[\int_{-\infty}^{+\infty} \cdots d x^{\prime} d y^{\prime}\right]\left[\int_{-\infty}^{+\infty} \cdots d x^{\prime \prime} d y^{\prime \prime}\right]^{*} e^{j \frac{2 \pi}{\lambda z}(x u+y v)} d u d v \\
= & \frac{1}{\lambda^{2} z^{2}} \int_{-\infty}^{+\infty} d x^{\prime} d y^{\prime} \mathbf{U}_{o}\left(x^{\prime}, y^{\prime}\right) \int_{-\infty}^{+\infty} d x^{\prime \prime} d y^{\prime \prime} \mathbf{U}_{o}^{*}\left(x^{\prime \prime}, y^{\prime \prime}\right) \\
& \times \underbrace{\int_{-\infty}^{+\infty} d u d v \exp \left\{-j \frac{2 \pi}{\lambda z}\left[u\left(-x+x^{\prime}-x^{\prime \prime}\right)+v\left(-y+y^{\prime}-y^{\prime \prime}\right)\right]\right\}}_{\delta\left(\frac{-x+x^{\prime}-x^{\prime \prime}}{\lambda z}, \frac{-y+y^{\prime}-y^{\prime \prime}}{\lambda z}\right)}
\end{aligned}
$$

Noting that $\delta(a x)=\frac{1}{|a|} \delta(x)$, Eq.(C.5) evaluates to

$$
\begin{aligned}
\mathbf{A}_{U_{o}}(x, y) & =\int_{-\infty}^{+\infty} d x^{\prime} d y^{\prime} \mathbf{U}_{o}\left(x^{\prime}, y^{\prime}\right) \int_{-\infty}^{+\infty} d x^{\prime \prime} d y^{\prime \prime} \mathbf{U}_{o}^{*}\left(x^{\prime \prime}, y^{\prime \prime}\right) \delta\left(-x+x^{\prime}-x^{\prime \prime},-y+y^{\prime}-y^{\prime \prime}\right) \\
& =\int_{-\infty}^{+\infty} \int_{o}\left(x^{\prime}, y^{\prime}\right) \mathbf{U}_{o}^{*}\left(x^{\prime}-x, y^{\prime}-y\right) d x^{\prime} d y^{\prime}
\end{aligned}
$$

Therefore, it is shown that the inverse Fourier transform of the far-field intensity distribution, $\left|\mathbf{U}_{1}\right|^{2}$, is equal to the auto-correlation of the aperture field distribution, i.e.

$$
\iint_{-\infty}^{+\infty}\left|\mathbf{U}_{1}(u, v)\right|^{2} e^{j \frac{2 \pi}{\lambda z}(x u+y v)} d u d v=\iint_{-\infty}^{+\infty} \mathbf{U}_{o}\left(x^{\prime}, y^{\prime}\right) \mathbf{U}_{o}^{*}\left(x^{\prime}-x, y^{\prime}-y\right) d x^{\prime} d y^{\prime} .
$$

Note that the Fourier transform of $\left|\mathbf{U}_{1}(u, v)\right|^{2}$ is $\mathbf{A}_{U_{o}}(-x,-y)$.

\section{C.2.3 Aperture geometry}

To achieve retrieval, the aperture geometry has to be arranged such that

$$
\mathbf{U}_{o}(x, y)=\delta(x, y)+\mathbf{S}(x, y)
$$


where $\mathbf{S}(x, y)$ is the quantity of interest. The term $\mathbf{S}(x, y)$ therefore exists in the resultant auto-correlation of $\mathbf{U}_{o}(x, y)$. In practice, $\mathbf{S}(x, y)$ has to be sufficiently separated spatially from the $\delta(x, y)$ function in order for the auto-correlation to isolate the field quantity of interest, $\mathbf{S}(x, y)$. 


\section{Appendix D}

\section{Phase grating}

A phase grating is an optical element whose "grating" functionality modulates the $a$ of the incident field. As a comparison, an amplitude grating modulates the $a$ of the incident field.

\section{D.1 $\pi$-phase-shift grating}

A $\pi$-phase-shift grating is the most commonly used phase grating, defined by

$$
\Pi(x)=\exp \{j \pi G(x)\}
$$

where

$$
G(x)=\frac{1}{2}+\frac{1}{2} \operatorname{sgn}\left[\sin \frac{2 \pi x}{d}+\sin \left(\frac{s-d / 2}{2}\right)\right]
$$

The period of this phase grating is denoted by $d$ and $s$ is the length of the grating tooth. Note that $0 \leq s \leq d$. Figure D.1(a) shows the amplitude and phase of the transmission function of this $\pi$-phase-shift grating. The phase change imposed by this $\pi$-phase-shift grating is alternating between $\pi$ and 0 , with the span of $\pi$-shift being $s$. For comparison, Fig. D.1(b) shows the transmission function of an amplitude grating with identical period $d$ and tooth length $s$. 

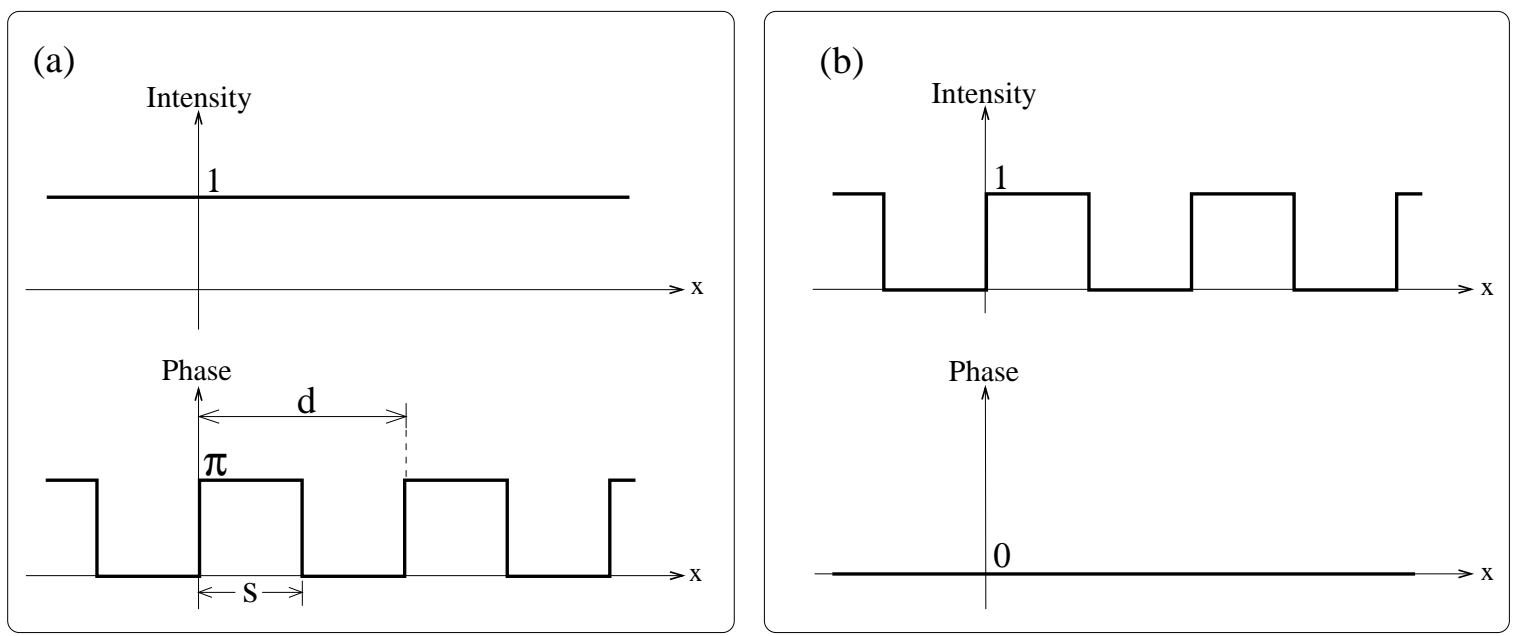

Figure D.1: (a) $\pi$-phase-shift grating. (b)Binary amplitude grating.

\section{D.1.1 Fourier series expansion}

Since this $\pi$-phase-shift grating is a periodic structure, its Fourier series expansion can be written as

$$
\Pi(x)=\exp \{j \pi G(x)\}=\sum_{n=-\infty}^{+\infty} a_{n} e^{j 2 \pi n f_{o} x}
$$

where the fundamental frequency $f_{o}$ is given by

$$
f_{o}=\frac{1}{d}
$$

and the Fourier coefficients $a_{n}$ is obtained by

$$
\begin{aligned}
a_{n} & =\frac{1}{d} \int_{d} \Pi(x) e^{-j 2 \pi n f_{o} x} d x \\
& =\frac{1}{d} \int_{0}^{d} \exp \{j \pi G(x)\} e^{-j 2 \pi n f_{o} x} d x \\
& =\frac{1}{d} \int_{0}^{s} e^{j \pi} e^{-j 2 \pi n f_{o} x} d x+\frac{1}{d} \int_{s}^{d} 1 \cdot e^{-j 2 \pi n f_{o} x} d x
\end{aligned}
$$

Case $\mathbf{n}=\mathbf{0}$ For $n=0$, the zeroth order coefficient $a_{0}$ is given by

$$
a_{0}=1-2\left(\frac{s}{d}\right)
$$


Case $\mathbf{n} \neq \mathbf{0} \quad$ For all other $n \neq 0$ terms, the $n$-th order coefficient $a_{n}$ can be obtained by

$$
\begin{aligned}
a_{n} & =\left.\frac{e^{j \pi}}{d} \frac{-1}{j 2 \pi n f_{o}} e^{-j 2 \pi n f_{o} x}\right|_{x=0} ^{x=s}+\left.\frac{1}{d} \frac{-1}{j 2 \pi n f_{o}} e^{-j 2 \pi n f_{o} x}\right|_{x=s} ^{x=d} \\
& =\frac{-1}{j \pi n}\left[1-e^{-j 2 \pi n(s / d)}\right]
\end{aligned}
$$

Fourier coefficients To conclude, the Fourier coefficients of a $\pi$-phase-shift grating is given by

$$
\left\{\begin{array}{l}
a_{0}=1-2 \vartheta \\
a_{n}=\frac{-1}{j \pi n}\left[1-e^{-j 2 \pi n \vartheta}\right] \quad \text { for } \quad n= \pm 1, \pm 2, \pm 3, \cdots
\end{array}\right.
$$

where

$$
\vartheta=\frac{s}{d}
$$

is the duty-cycle of the grating. Note that when the duty-cycle $\vartheta$ equals to 0.5 , even orders $(n=0, \pm 2, \cdots)$ disappear.

\section{D.2 Efficiency of $\pi$-phase-shift grating}

The relative strength of the diffraction orders from a $\pi$-phase-shift grating is proportional to the modulus square of its Fourier coefficients. As will be shown later by the Parseval's theorem, the summation of the modulus square of the Fourier coefficients over all the diffracted orders of a $\pi$-phase-shift grating equals to 1 . Considering that the $\pi$-phase-shift grating here does not have any absorption, the diffraction efficiency is simply the modulus square of the Fourier coefficients.

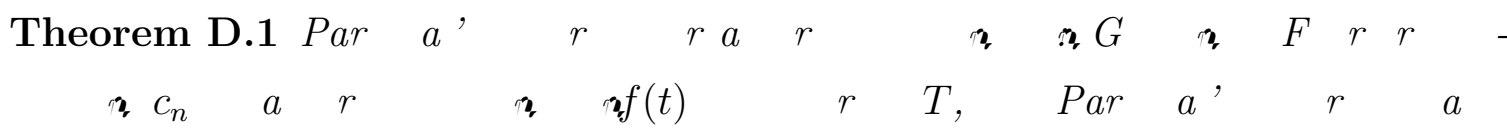

$a$

$$
\frac{1}{T} \int_{T}|f(t)|^{2} d t=\sum_{n=-\infty}^{+\infty}\left|c_{n}\right|^{2}
$$




\section{Proof}

The Fourier series decomposition of the periodic function $f(t)$ can be written as

$$
f(t)=\sum_{n=-\infty}^{+\infty} c_{n} e^{j 2 \pi n t / T}
$$

Note that

$$
f(t) f^{*}(t)=\sum_{n=-\infty}^{+\infty} \sum_{m=-\infty}^{+\infty} c_{n} c_{m}^{*} e^{j 2 \pi(n-m) t / T}
$$

and integrate both sides of the above equation over one period $T$

$$
\int_{T}|f(t)|^{2} d t=\sum_{m=-\infty}^{+\infty} \sum_{n=-\infty}^{+\infty} c_{m} c_{n}^{*} \underbrace{\int_{T} e^{j 2 \pi(m-n) t / T} d t}_{T \delta_{m n}}
$$

Applying the Parseval's theorem to Eq.(D.3), i.e. the transmission function of the periodic $\pi$-phase-shift grating, one finds

$$
\frac{1}{d} \int_{d}|\Pi(x)|^{2} d x=\sum_{n=-\infty}^{+\infty}\left|a_{n}\right|^{2}=1
$$

Therefore, the diffraction efficiency of each diffracted order from the $\pi$-phase-shift grating is simply the modulus square of its Fourier coefficient, $\left|a_{n}\right|^{2}$.

Using the following identity,

$$
\left|1-e^{j \varphi}\right|=\sqrt{(1-\cos \varphi)^{2}+\sin ^{2} \varphi}=\sqrt{2(1-\cos \varphi)}=\sqrt{2\left(2 \sin ^{2} \frac{\varphi}{2}\right)}
$$

the diffraction efficiency of a $\pi$-phase-shift grating is

$$
\eta_{n}=\left|a_{n}\right|^{2}=\left\{\begin{array}{lll}
|1-2 \vartheta|^{2} & \text { for } & n=0 \\
\frac{4 \sin ^{2} \pi n \vartheta}{\pi^{2} n^{2}} & \text { for } & n= \pm 1, \pm 2, \pm 3, \cdots .
\end{array}\right.
$$

\section{D.2.1 Efficiency of a 50\% duty-cycle $\pi$-phase-shift grating}

For a $50 \%$ duty-cycle $\pi$-phase-shift grating, i.e. $\vartheta=s / d=0.5$, its diffraction efficiency is given by

$$
\eta_{n}=\left|a_{n}\right|^{2}=\left\{\begin{array}{cl}
\frac{4}{\pi^{2} n^{2}} & \text { for } n= \pm 1, \pm 3, \pm 5, \cdots \\
0 & \text { otherwise }
\end{array}\right.
$$


This $50 \%$ duty-cycle $\pi$-phase-shift grating has only odd diffraction orders. This is a desirable property in many applications since there is no strong zeroth order present in its far-field diffraction pattern. 


\section{Bibliography}

[1] M. Born and E. Wolf, $\operatorname{Pr} \quad O \quad$ (Cambridge University Press, UK, 1997), 6th edition.

[2] J. W. Goodman, I F r r $\quad$ (McGraw-Hill, New York, 1996), 2nd edition.

[3] J. W. Goodman, $S$ a $\quad$ a $O \quad$ (Wiley, New York, 1985).

[4] N. Bloembergen, "Nonlinear optics-past, present, and future," IEEE J. $Q$ a E $\quad r \quad 20(6), 556-8(1984)$.

[5] B. J. Thompson and E. Wolf, "Two-beam interference with partially coherent light," J. O . S . A . 47, 895-902 (1957).

[6] F. Zernike, "The concept of degree of coherence and its application to optical problems," $P \quad a \mathrm{~V}(8), 785-95$ (1938).

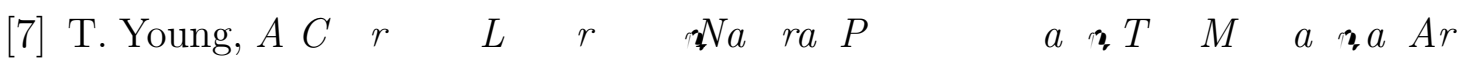
$I$ (Printed for J. Johnson, London, 1807).

[8] D. T. Attwood, $S \quad X-R a \quad a \quad E \quad r \quad U$ ra $\quad$ ra $\quad$ Cambridge University Press, UK, 1999).

[9] C. Chang, P. Naulleau, E. Anderson, and D. Attwood, "Spatial coherence characterization of undulator radiation," $O$. C 182(1-3), 25-34 (2000). 
[10] C. Chang, E. Anderson, P. Naulleau, E. M. Gullikson, K. A. Goldberg, and D. Attwood, "Direct measurement of index of refraction in the extremeultraviolet wavelength region with a novel interferometer," $O \quad L \quad$. 27(12), 1028-30 (2002).

[11] C. Chang, P. Naulleau, E. Anderson, K. Rosfjord, and D. Attwood, "Diffractive optical elements based on fourier optical techniques: A new class of optics for extreme ultraviolet and soft x-ray wavelengths," $A$. $O$. (Cover feature, December 2002).

[12] D. L. Abernathy, G. Gruebel, S. Brauer, I. McNulty, G. B. Stephenson, S. G. J. Mochrie, $\quad a$., "Small-angle x-ray scattering using coherent undulator radiation at the ESRF," J. $S$ r $r$ r Ra a . 5(1), 37-47 (1998).

[13] D. T. Attwood, K. Halbach, and K. J. Kim, "Tunable coherent x-rays," $S$ 228, 1265-72 (1985).

[14] R. Coïsson, "Spatial coherence of synchrotron radiation," $A \quad$. O . 34(5), 9048 (1995).

[15] Z. W. Hu, P. A. Thomas, A. Snigirev, I. Snigireva, A. Souvorov, P. G. R. Smith, $a$., "Phase-mapping of periodically domain-inverted $\mathrm{LiNbO}_{3}$ with coherent X-rays," Na r 392, 690-3 (1998).

[16] A. M. Kondratenko and A. N. Skrinsky, "Use of radiation of electron storage rings in x-ray holography of objects," $O \quad . S \quad r \quad$.42(2), 338-44 (1977).

[17] R. Tai, Y. Takayama, N. Takaya, T. Miyahara, S. Yamamoto, H. Sugiyama, $a$., "A novel intensity interferometer for synchrotron radiation in the vacuum ultraviolet and soft x-ray regions," $R$. S . I r . 71(3), 1256-63 (2000).

[18] Y. Takayama, R. Z. Tai, T. Hatano, T. Miyahara, W. Okamoto, and Y. Kagoshima, "Measurement of the coherence of synchrotron radiation," $J$. $S \quad r \quad r \quad R a \quad a .5(3), 456-8$ (1998). 
[19] D. Weiss, G. Schneider, B. Niemann, P. Guttmann, D. Rudolph, and G. Schmahl, "Computed tomography of cryogenic biological specimens based on x-ray microscopic images," U ra $\quad r \quad$ 84(3-4), 185-97 (2000).

[20] W. Meyer-Ilse, G. Denbeaux, L. E. Johnson, W. Bates, A. Lucero, and E. H. Anderson, "The high resolution x-ray microscope, XM-1," in "Sixth International Conference on X-Ray Microscopy, Berkeley, CA, USA 1999," (AIP Conf. Proc., 507), pp. 129-34.

[21] P. Fischer, T. Eimuller, G. Schutz, G. Denbeaux, A. Pearson, L. Johnson, a ., "Element-specific imaging of magnetic domains at $25 \mathrm{~nm}$ spatial resolution using soft x-ray microscopy," $R$. S . I r . 72(5), 2322-4 (2001).

[22] E. H. Anderson, D. L. Olynick, B. Harteneck, E. Veklerov, G. Denbeaux, W. Chao, a., "Nanofabrication and diffractive optics for high-resolution $\mathrm{x}$ ray applications," J. Va . S . T . B 18, 2970-5 (2000).

[23] H. Ade and B. Hsiao, "X-ray linear dichroism microscopy," $S \quad$ 262, 1427-9 (1993).

[24] K. M. Kemner, W. Yun, Z. Cai, B. Lai, H. R. Lee, J. Maser, a ., "Using zone plates for x-ray microimaging and microspectroscopy in environmental science," $J . S \quad r \quad r \quad R a \quad a .6(3), 639-41$ (1999).

[25] J. Stöhr, M. G. Samant, J. Luning, A. C. Callegari, P. Chaudhari, J. P. Doyle, a., "Liquid crystal alignment on carbonaceous surfaces with orientational order," $S$ 292, 2299-302 (2001).

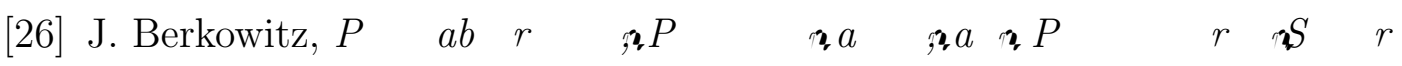
(Academic Press, New York, 1979).

[27] J. Walker, A. B. C., J. Barbee, T. W., R. B. Hover, and J. F. Lindblom, "Soft X-ray images of the solar corona with a normal-incidence Cassegrain multilayer telescope," $S$ 241, 1781-7 (1988). 
[28] J. Andruszkow, B. Aune, V. Ayvazyan, N. Baboi, R. Bakker, V. Balakin, a ., "First observation of self-amplified spontaneous emission in a free-electron laser at $109 \mathrm{~nm}$ wavelength," $P \quad . R \quad$. $L \quad$. 85(18), 3825-9 (2000).

[29] M. A. Kumakhov, "Capillary optics and their use in x-ray analysis," $X-R a$ $S \quad r \quad .29(5), 343-8(2000)$.

[30] A. Snigirev, V. Kohn, I. Snigireva, and B. Lengeler, "A compound refractive lens for focusing high-energy x-rays," $N a \quad r \quad 384,49-51$ (1996).

[31] L. Kipp, M. Skibowski, R. L. Johnson, R. Berndt, R. Adelung, S. Harm, a ., "Sharper images by focusing soft X-rays with photon sieves," $N a \quad r \quad \mathbf{4 1 4}, 184-8$ (2001).

[32] D. Paterson, B. E. Allman, P. J. McMahon, J. Lin, N. Moldovan, K. A. Nugent, $\quad a$., "Spatial coherence measurement of x-ray undulator radiation," $O$. C 195(1-4), 79-84 (2001).

[33] J. M. Heck, D. T. Attwood, W. Meyer-Ilse, and E. H. Anderson, "Resolution determination in x-ray microscopy: an analysis of the effects of partial coherence and illumination spectrum," J. X-Ra S . T . 8(2), 95-104 (1998).

[34] B. Niemann, P. Guttmann, D. Hambach, G. Schneider, D. Weiss, and G. Schmahl, "The condenser-monochromator with dynamical aperture synthesis for the TXM at an undulator beamline at BESSY II," in "Sixth International Conference on X-Ray Microscopy, Berkeley, CA, USA 1999," (AIP Conf. Proc., 507), pp. 440-5.

[35] D. T. Attwood, P. Naulleau, K. A. Goldberg, E. Tejnil, C. Chang, R. Beguiristain, $\quad a$., "Tunable coherent radiation in the soft x-ray and extreme ultraviolet spectral regions," IEEE J. Q a $\quad$ e $\quad r \quad$ a35(5), 709-20 (1999).

[36] H. M. Nussenzveig, $C a \quad a \quad a \quad D \quad r \quad R \quad a \quad$ (Academic Press, New York, 1972). 
[37] Y. Liu, M. Seminario, F. G. Tomasel, C. Chang, J. J. Rocca, and D. T. Attwood, "Achievement of essentially full spatial coherence in a high-average-power softx-ray laser," $P \quad$. R . A 63(3), 033802 (2001).

[38] M. C. Marconi, J. L. A. Chilla, C. H. Moreno, B. R. Benware, and J. J. Rocca, "Measurement of the spatial coherence buildup in a discharge pumped table-top soft x-ray laser," $P \quad . R \quad$. L . 79(15), 2799-802 (1997).

[39] D. Schulze, M. Dorr, G. Sommerer, J. Ludwig, P. V. Nickles, T. Schlegel, a ., "Polarization of the 61st harmonic from 1053-nm laser radiation in neon," $P$. R . A 57(4), 3003-7 (1998).

[40] C. Spielmann, H. Burnett, R. Sartania, R. Koppitsch, M. Schnurer, C. Kan, a., "Generation of coherent $\mathrm{x}$-rays in the water window using 5 -femtosecond laser pulses," $S$ 278, 661-4 (1997).

[41] K. A. Goldberg, P. Naulleau, and J. Bokor, "Extreme ultraviolet interferometric measurements of diffraction-limited optics," J. Va .S . T . B 17(6), 29826 (1999).

[42] P. Naulleau, K. A. Goldberg, S. H. Lee, C. Chang, D. Attwood, and J. Bokor, "Extreme-ultraviolet phase-shifting point-diffraction interferometer: a wavefront metrology tool with subangstrom reference-wave accuracy," $A$. 38(35), 7252-63 (1999).

[43] E. Tejnil, K. A. Goldberg, and J. Bokor, "Phase effects owing to multilayer coatings in a two-mirror extreme-ultraviolet Schwarzschild objective," $A \quad . O$. 37(34), 8021-9 (1998).

[44] M. Takeda, H. Ina, and S. Kobayashi, "Fourier-transform method of fringepattern analysis for computer-based topography and interferometry," J. $O$. $S$. A . 72(1), 156-60 (1982).

[45] P. Naulleau, K. A. Goldberg, E. M. Gullikson, and J. Bokor, "At-wavelength, 
system-level flare characterization of extreme-ultraviolet optical systems," $A$. O . 39(17), 2941-7 (2000).

[46] P. Naulleau and K. A. Goldberg, "Dual-domain point diffraction interferometer," A . O . 38(16), 3523-33 (1999).

[47] W. Leitenberger, S. M. Kuznetsov, and A. Snigirev, "Interferometric measurements with hard x-rays using a double slit," $O$. $C$ 191(1-2), 91-6 (2001).

[48] V. N. Mahajan, Ab rra $\quad r \quad M a \quad S \quad$ (SPIE Press, Bellingham WA, 1991).

[49] B. R. A. Nijboer, $T$ D ra $T$ T Abra Ph.D. dissertation, Groningen University, the Netherlands (1942).

[50] D. Joyeux, F. Polack, and D. Phalippou, "An interferometric determination of the refractive part of optical constants for carbon and silver across soft x-ray absorption edges," $R . S . I \quad r \quad .70(7), 2921-6$ (1999).

[51] U. Bonse and M. Hart, "An x-ray interferometer," $A \quad$. $P \quad . L \quad$. 6(8), 155-6 (1965).

[52] H. Ehrenreich and H. R. Philipp, "Optical properties of $\mathrm{Ag}$ and $\mathrm{Cu}$," $P \quad R$. 128, 1622-9 (1962).

[53] E. M. Gullikson, P. Denham, S. Mrowka, and J. H. Underwood, "Absolute photoabsorption measurements of $\mathrm{Mg}, \mathrm{Al}$, and $\mathrm{Si}$ in the soft-x-ray region below the $L_{2,3}$ edges," $P \quad$. R . B 49(23), 16283-8 (1994).

[54] H. J. Hagemann, W. Gudat, and C. Kunz, "Optical constants from the far infrared to the x-ray region: $\mathrm{Mg}, \mathrm{Al}, \mathrm{Cu}, \mathrm{Ag}, \mathrm{Au}, \mathrm{Bi}, \mathrm{C}$, and $\mathrm{Al}_{2} \mathrm{O}_{3}, " J . O$. $S . A .65(6), 742-4(1975)$. 
[55] E. Shiles, T. Sasaki, M. Inokuti, and D. Y. Smith, "Self-consistency and sum-rule tests in the Kramers-Kronig analysis of optical data: Applications to aluminum," $P \quad . R$.B 22(4), 1612-28 (1980).

[56] R. Soufli, $O \quad a C$ a $\quad M a$ ra $\quad E U V / S \quad X-R a \quad R \quad r M$ a $\operatorname{rrr} \operatorname{rr} \quad a \quad$ Ph.D. dissertation, University of California, Berkeley (1997).

[57] R. Soufli and E. M. Gullikson, "Reflectance measurements on clean surfaces for the determination of optical constants of silicon in the extreme ultraviolet/softx-ray region," A . O . 36(22), 5499-507 (1997).

[58] R. Soufli and E. M. Gullikson, "Absolute photoabsorption measurements of molybdenum in the range $60-930 \mathrm{eV}$ for optical constant determination," $A$. O . 37(10), 1713-19 (1998).

[59] W. R. Hunter, "Errors in using the reflectance vs angle of incidence method for measuring optical constants," J. O . S . A . 55(10), 1197-1204 (1965).

[60] W. R. Hunter, "Measurement of optical properties of materials in the vacuum ultraviolet spectral region," $A \quad$. O . 21(12), 2103-14 (1982).

[61] D. L. Windt, "XUV optical constants of single-crystal GaAs and sputtered C, Si, $\mathrm{Cr}_{3} \mathrm{C}_{2}$, Mo, and W," $A$. O . 30(1), 15-25 (1991).

[62] D. L. Windt, J. Cash, W. C., M. Scott, P. Arendt, B. Newnam, R. F. Fisher, a., "Optical constants for thin films of C, Diamond, Al, Si, and CVD SiC from $24 \AA$ to $1216 \AA, " A \quad$. O . 27(2), 279-95 (1988).

[63] E. D. Palik and G. Ghosh, "The electronic handbook of optical constants of solids," (1999), 1 computer compact disc.

[64] E. M. Gullikson, http://www.cxro.1bl.gov/optical_constants/.

[65] H. Medecki, E. Tejnil, K. A. Goldberg, and J. Bokor, "Phase-shifting point diffraction interferometer," $O$. L . 21(19), 1526-8 (1996). 
[66] S. H. Lee, P. Naulleau, K. A. Goldberg, C. H. Cho, S. T. Jeong, and J. Bokor, "Extreme-ultraviolet lensless Fourier-transform holography," $A$. O . 40(16), 2655-61 (2001).

[67] W. Leitenberger and A. Snigirev, "Microscopic imaging with high energy x-rays by Fourier transform holography," J. A . P . 90(2), 538-44 (2001).

[68] A. C. Price, L. B. Sorensen, S. D. Kevan, J. Toner, A. Poniewierski, and R. Holyst, "Coherent soft-x-ray dynamic light scattering from smectic-A films," $P \quad . R \quad . L \quad$. 82(4), 755-8 (1999).

[69] A. Fera, I. P. Dolbnya, G. Grubel, H. G. Muller, B. I. Ostrovskii, A. N. Shalaginov, $\quad a$. ., "Complex dynamic behavior of fluctuating smectic-A films as studied by scattering with coherent x-rays," $P \quad$. $R \quad$. $L \quad$. 85(11), 2316-19 (2000).

[70] C. Riekel, "New avenues in x-ray microbeam experiments," $R . P r . P$. 63(3), 233-62 (2000). 